\title{
Stellar Rotation in the Gaia Era: Revised Open Clusters' Sequences
}

\author{
Diego Godoy-Rivera $^{1}$ (D), Marc H. Pinsonneault ${ }^{1}$ (D), and Luisa M. Rebull ${ }^{2}$ (D) \\ ${ }^{1}$ Department of Astronomy, The Ohio State University, 140 W. 18th Ave., Columbus, OH 43210, USA; godoyrivera.1@ osu.edu \\ ${ }^{2}$ Infrared Science Archive (IRSA), IPAC, 1200 E. California Blvd., California Institute of Technology, Pasadena, CA 91125, USA \\ Received 2020 December 21; revised 2021 July 30; accepted 2021 August 22; published 2021 November 29
}

\begin{abstract}
The period versus mass diagrams (i.e., rotational sequences) of open clusters provide crucial constraints for angular momentum evolution studies. However, their memberships are often heavily contaminated by field stars, which could potentially bias the interpretations. In this paper, we use data from Gaia DR2 to reassess the memberships of seven open clusters with ground- and space-based rotational data, and present an updated view of stellar rotation as a function of mass and age. We use the Gaia astrometry to identify the cluster members in phase space, and the photometry to derive revised ages and place the stars on a consistent mass scale. Applying our membership analysis to the rotational sequences reveals that: (1) the contamination in clusters observed from the ground can reach up to $\sim 35 \%$; (2) the overall fraction of rotational outliers decreases substantially when the field contaminants are removed, but some outliers persist; (3) there is a sharp upper edge in the rotation periods at young ages; (4) at young ages, stars in the $1.0-0.6 M_{\odot}$ range inhabit a global maximum of rotation periods, potentially providing an optimal window for habitable planets. Additionally, we see clear evidence for a strongly mass-dependent spindown process. In the regime where rapid rotators are leaving the saturated domain, the rotational distributions broaden (in contradiction with popular models), which we interpret as evidence that the torque must be lower for rapid rotators than for intermediate ones. The cleaned rotational sequences from ground-based observations can be as constraining as those obtained from space.
\end{abstract}

Unified Astronomy Thesaurus concepts: Open star clusters (1160); Star clusters (1567); Stellar ages (1581); Stellar rotation (1629); Low mass stars (2050)

Supporting material: machine-readable tables

\section{Introduction}

Together with mass and metallicity, rotation is one of the defining properties of stars. Stellar rotation is strongly massdependent (Kraft 1967a; García et al. 2014), and while stars with radiative envelopes $\left(M \gtrsim 1.4 M_{\odot}\right)$ live their lives as rapid rotators, stars with convective envelopes spin down as they age (Skumanich 1972). The overall framework of the rotational evolution of low-mass stars is that they are born with a range of rotation rates ( $\sim 1-10$ days; Herbst et al. 2002) and star-disk coupling timescales (from $<1$ and up to $\sim 10 \mathrm{Myr}$; Williams \& Cieza 2011), and once they arrive onto the zero-age mainsequence (ZAMS), they experience a decrease in their rotational velocities due to angular momentum losses caused by magnetized winds (Parker 1958; Schatzman 1962; Weber \& Davis 1967; Kawaler 1988; Pinsonneault et al. 1989).

For lower main-sequence stars, the observable stellar properties do not appreciably change with time, which makes the determination of ages of individual stars challenging (Soderblom 2010). In this regard, the empirical spin-down of low-mass stars offers a valuable age diagnostic, and this idea is known as gyrochronology (Barnes 2003, 2007; Mamajek \& Hillenbrand 2008).

If properly validated, gyrochronology can provide valuable age information for Galactic and stellar population studies (McQuillan et al. 2013, 2014; Davenport 2017; Davenport \& Covey 2018; van Saders et al. 2019), as well as for the characterization of exoplanet hosts (e.g., Gallet \& Delorme 2019; Gallet 2020; Carmichael et al. 2021; David et al. 2021; Zhou et al. 2021). Moreover, although stellar evolution theory is successful in predicting numerous properties of stars, this is not the case for the evolution of angular momentum and its related physical processes (e.g., transport in stellar interiors, birth conditions, magnetic braking). Consequently, stellar models that incorporate it need to be calibrated with empirical observations (e.g., Gossage et al. 2021).

All of the above contribute to a compelling need for gyrochronology to be examined and tested. For old ages ( few Gyr), these efforts have used field stars (Irwin et al. 2011; Angus et al. 2015; Newton et al. 2016; van Saders et al. 2016; Lorenzo-Oliveira et al. 2019) and wide-separation binaries (Chanamé \& Ramírez 2012; Janes 2017; Godoy-Rivera \& Chanamé 2018; Otani et al. 2021). For young ages $(\lesssim 1 \mathrm{Gyr})$, the calibrations have been based on the empirical rotational sequences (i.e., period versus mass or temperature diagrams) of star-forming regions, associations, and open clusters (Denissenkov et al. 2010; Gallet \& Bouvier 2013, 2015; Angus et al. 2019; de Freitas 2021).

With the advent of space-based missions, the field of stellar rotation is entering a new era. The unparalleled astrometry from the Gaia mission (Gaia Collaboration et al. 2018b) has already allowed new gyrochronology inspections to be carried out (e.g., Curtis et al. 2019b; Angus et al. 2020). In addition to this, results obtained from the observations by the Kepler and K2 missions (Borucki et al. 2010; Howell et al. 2014) have showed striking trends at various ages. These include strongly massdependent rotation rates in populations younger than $10 \mathrm{Myr}$ (Somers et al. 2017; Rebull et al. 2018, 2020), unusual spindown behavior in $\sim 1-3$ Gyr old clusters (Curtis et al. 2019a, 2020; Gruner \& Barnes 2020; see also Agüeros et al. 2018 ), and anomalously rapid rotation in $\gtrsim 5$ Gyr old field stars (van Saders et al. 2016; Hall et al. 2021).

The strengths of space data, namely unparalleled time coverage and photometric precision, have indeed provided 
exquisite data in selected regimes. However, for many interesting clusters, a combination of crowded fields, faint sources, and long rotation periods make obtaining data from space surveys impractical. By contrast, ground-based surveys can measure precise and accurate rotation periods for even faint sources, because starspot modulation induces relatively large photometric signals. The native seeing even from average sites is also far better than the $4^{\prime \prime}$ pixels of Kepler and K2, or the $21^{\prime \prime}$ pixels of the Transiting Exoplanet Survey Satellite (TESS; Ricker et al. 2015). As a result, ground-based surveys are still fundamental for studies of stellar rotation.

In this context, although star clusters have played a crucial role in our understanding of angular momentum evolution, a thorough decontamination of their rotational sequences is currently lacking. For the systems that have been observed from the ground, the field contamination in their sequences is expected to go from $\sim 20 \%$ (e.g., Hartman et al. 2009b) and reach up to $\sim 40 \%-60 \%$ (e.g., Irwin et al. 2007b, 2008, 2009). For the systems observed from space, a lower contamination rate may be expected due to more careful target selections, but given their importance as rotational benchmarks, detailed astrometric analyses are certainly needed. Furthermore, it has been common in the literature to assume that rotational outliers of cluster sequences correspond to field contaminants. We are now in a position to test this assumption.

Ultimately, the unknown extent to which contamination is a problem, and what effect this could have on the patterns of the rotational sequences, make the revision of their memberships an imperative task. This is something the unprecedented spacebased data from Gaia is particularly well-suited for. With this, the goal of this paper is to use the high-precision Gaia astrometry to clean the rotational sequences of a sample of open clusters (observed from the ground and space), with the prospects of providing revised gyrochronology calibrators, and an updated view of stellar rotation as a function of mass and age.

This paper is structured as follows. In Section 2, we present the sample of star clusters with rotation period measurements we study. In Section 3, we present our method for identifying likely cluster members using the Gaia astrometry. In Section 4, we revise the properties of the clusters we study, and calculate stellar masses and temperatures for their members. In Section 5, we show the effects that the membership analysis has on the rotational sequences. In Section 6, we discuss our findings and present an updated view of stellar rotation. We conclude in Section 7.

\section{Sample Selection and Rotation Period Data}

We compile a list of open clusters that satisfy four conditions: (1) they hold high scientific interest for constraining the processes that govern stellar rotation; (2) they have rotation period information, derived uniformly within each cluster, for w hundreds of candidate members; (3) they have reliable Gaia data that allow us to accurately model them astrometrically; (4) they potentially have enough nonmember contamination that a careful membership analysis to clean their rotational sequences would have significant impact. The rotation periods we use come from both ground- and space-based optical photometric monitoring. Due to the target selections of the original rotation period references, the membership contamination is expected to be higher for the clusters observed from the ground than for
Table 1

List of the Seven Open Clusters We Study

\begin{tabular}{lcc}
\hline \hline Name & $\begin{array}{c}\text { Literature Age (pre-Gaia) } \\
(\text { Myr })\end{array}$ & $N_{\star, \text { Period }}$ \\
\hline NGC 2547 & 35 & 176 \\
Pleiades & 125 & 759 \\
M50 & 130 & 812 \\
NGC 2516 & 150 & 362 \\
M37 & 550 & 367 \\
Praesepe & 580 & 809 \\
NGC 6811 & 1000 & 235 \\
\hline
\end{tabular}

Note. The clusters are sorted by increasing age. The quoted ages come from the compilation by Gallet \& Bouvier (2015) and are the representative pre-Gaia values. The period measurements are taken from the references cited throughout Section 2.

those observed from space (see the clusters' descriptions below).

We limit our selection to open clusters but do not include star-forming regions and associations, which require a more complex treatment of membership (e.g., Gagné \& Faherty 2018; Galli et al. 2018; Kuhn et al. 2019). We also exclude systems that are either too close or too distant for a reliable membership analysis, or that have poor Gaia data, or systems with smaller or heterogeneous rotation samples. We discuss the systems with available rotational data sets that we did not analyze in Appendix A.

Table 1 lists the seven open clusters we study, ${ }^{3}$ together with the number of stars in each of them with period measurements. Table 1 also reports their literature ages as compiled by Gallet \& Bouvier (2015) (see their Table 1 for the specific references), but we highlight the fact that, for our analysis, we perform an independent derivation of revised cluster properties using Gaia data and recent spectroscopic results (see Section 4). We now describe the seven clusters in our sample.

\subsection{Three Monitor Project Clusters}

The Monitor Project ${ }^{4}$ performed an optical photometric survey of several young open clusters using 2 and $4 \mathrm{~m}$ class telescopes with wide field cameras (Irwin et al. 2007b). The derived lightcurves allowed for searches of transiting planets (Miller et al. 2008) and eclipsing binaries (Irwin et al. 2007a), as well as measurements of rotation periods.

Of the clusters observed by the Monitor Project, we include NGC 2547, M50, and NGC 2516 in our sample. All three of these clusters offer interesting constraints on stellar rotation evolution. With an age of 35 Myr, NGC 2547 is a well-populated system where solar analogs have just arrived on the main sequence, and data in this age range (younger than the Pleiades and older than star-forming regions) is a sensitive test of theory. With ages of $\sim 130$ and 150 Myr, M50 and NGC 2516 offer natural comparison points for the benchmark Pleiades cluster. Additionally, the contamination rates quoted for these clusters are in the $\sim 40 \%$ $60 \%$ range (Irwin et al. 2007b, 2008, 2009), which hints that a revision of their rotational sequences could be significant.

\footnotetext{
3 Alternative names for some of our systems are: Pleiades $=$ M45 $=$ Melotte 22; $\mathrm{M} 50=\mathrm{NGC}$ 2323; $\mathrm{M} 37=\mathrm{NGC} 2099 ; \quad$ Praesepe $=\mathrm{M} 44=$ Melotte $88=$ NGC 2632; NGC $6811=$ Melotte 222. All throughout this paper, we refer to them with the names listed in Table 1.

4 https://www.ast.cam.ac.uk/ioa/research/monitor/
} 
NGC 2547, M50, and NGC 2516 were studied in a similar fashion by Irwin et al. (2008), Irwin et al. (2009), and Irwin et al. (2007b), respectively. These authors surveyed regions out to $\sim 50^{\prime}, \sim 30^{\prime}$, and $\sim 60^{\prime}$ from the clusters' center, identified candidate members on the basis of $V$ versus $V-I$ colormagnitude diagram (CMD) selections, and reported rotation periods for 176, 812, and 362 stars, respectively.

\subsection{One MMT Cluster}

M37 (age of $\sim 550 \mathrm{Myr}$ ) is one of the very few open clusters with a large rotational sample that are older than 500 Myr. This, in addition to its age being close to the classical $\sim 580 \mathrm{Myr}$ age for Praesepe, along with a quoted $\sim 20 \%$ contamination rate (Hartman et al. 2009b), make it an interesting system to include in our sample.

Hartman et al. (2008b) observed the M37 cluster with the 6.5 $\mathrm{m}$ MMT telescope in order to constrain its parameters, study variable stars (Hartman et al. 2008a), measure rotation periods (Hartman et al. 2009b), and study the occurrence rate of transiting planets (Hartman et al. 2009a). Given the exquisite optical observations, thorough lightcurve analysis, and the aforementioned expected contamination rate, we include M37 in our sample.

Hartman et al. (2009b) surveyed regions out to $\sim 20^{\prime}$ from the cluster center, identified candidate members on the basis of two CMD selections ( $r$ versus $g-r$ and $g-i$ ), and reported rotation periods for 575 stars. Of these, however, only 367 are considered by Hartman et al. (2009b) to be the clean periodic sample, where the different algorithms for determining periods showed good agreement with each other, and their results did not differ by more than $10 \%$. We take this clean sample as our nominal M37 data set.

\subsection{Three Kepler Clusters}

The Kepler spacecraft, in both its original $4 \mathrm{yr}$ mission and subsequent $\mathrm{K} 2$ mission, observed several open clusters of a range of ages. These observations produced lightcurves with exquisite photometric precision, and have been used to construct rotation period catalogs for a number of clusters.

Of the clusters observed by Kepler and K2, we include the Pleiades, Praesepe, and NGC 6811 in our sample. Given their proximity, the Pleiades (distance of $\approx 136 \mathrm{pc}$; age of $\sim 125$ Myr) and Praesepe (distance of $\approx 186 \mathrm{pc}$; age of $\sim 580 \mathrm{Myr}$ ) clusters have been used for rotation studies for several decades (e.g., Anderson et al. 1966; Kraft 1967b; Dickens et al. 1968; Stauffer et al. 1984, 2016; Stauffer \& Hartmann 1987; Queloz et al. 1998; Terndrup et al. 1999, 2000; Scholz \& Eislöffel 2007; Agüeros et al. 2011; Delorme et al. 2011; Douglas et al. 2014; Rebull et al. 2016a, 2016b, 2017), and the periods derived from the $\mathrm{K} 2$ observations comprise their state-of-theart rotational samples. While the number of NGC 6811 stars with Kepler periods is lower compared to the aforementioned clusters, its old age ( $\sim 1 \mathrm{Gyr})$ makes it a remarkably interesting system for stellar rotation studies (e.g., Meibom et al. 2011a; Curtis et al. 2019a; Rodríguez et al. 2020; Velloso et al. 2020).

1. Pleiades: Rebull et al. (2016a) performed a comprehensive lightcurve analysis of the stars observed by $\mathrm{K} 2$ in regions out to $\sim 350^{\prime}$ from the cluster center. Rebull et al. (2016b) expanded on this by meticulously classifying and identifying the different structures present in the periodograms and lightcurves, and Stauffer et al. (2016) then used this to study the Pleiades in the context of angular momentum evolution. The sample of candidate members used by these studies comprises 759 stars with measured periods that were classified as Best $+O K$ members by Rebull et al. (2016a) on the basis of proper motions and position in the CMD selections. We take this sample as our nominal Pleiades data set. We note that, although the $\mathrm{K} 2$ observations missed part of the northern region of the cluster, this is unlikely to bias the period distribution (Rebull et al. 2016a).

2. Praesepe: a study similar to that of the Pleiades was carried out for Praesepe by Rebull et al. (2017). In this case, the candidate members extend out to $\sim 400^{\prime}$ from the cluster center, and they were selected on the basis of proper motions and CMD position. The final Rebull et al. (2017) sample comprises 809 candidate members with measured periods.

3. NGC 6811: Meibom et al. (2011a) studied candidates that were previously vetted using radial velocity $(\mathrm{RV})$ data in a region out to $30^{\prime}$ from the cluster center, and reported periods for 71 stars. Curtis et al. (2019a) surveyed a region of $60^{\prime}$ radius, selected candidate members on the basis of Gaia CMD position and astrometry, and reported periods for 171 stars. We complement these with the Santos et al. $(2019,2021)$ Kepler field catalogs, which reported periods for 194 NGC 6811 candidate members. To maximize the number of stars with measured periods, we combine all of these catalogs and end up with 235 stars after accounting for repetitions. For stars in common among the references, we prioritize the Santos et al. (2019) and Santos et al. (2021) periods first, Curtis et al. (2019a) second, and Meibom et al. (2011a) third (although a comparison of these values showed excellent agreement). While the periods of our joint NGC 6811 catalog are derived from slightly different techniques, the studies carried out by these four references are all based on the same Kepler data.

\section{Membership Method}

In this section, we describe the method we use to analyze the clusters in our sample. In Section 3.1, taking NGC 2547 as a working example, we use the Gaia astrometry to fit the cluster and the field in phase space, and to calculate membership probabilities. Since the periodic samples are deep and include faint stars with large formal astrometric errors, we classify stars into four different groups: highly likely cluster members, highly likely nonmembers, an intermediate category of possible members (typically faint objects with large astrometric uncertainties), and a category of stars without enough information to be classified. We are interested in both single and binary stars, and while we attempt to avoid biasing our results against binaries, there are Gaia selection effects related to the excess astrometric noise from them (see Section 6). We do not apply a Hertzsprung-Russell (HR) diagram selection, but our final samples are strikingly clear in that plane. In Section 3.2, we crossmatch the NGC 2547 Gaia data with its periodic sample. In Section 3.3, we show the results of applying our membership method to all our clusters. 


\subsection{Gaia Data, Cluster Fitting, and Membership Probabilities}

In Section 3.1.1, we describe the Gaia data; in Section 3.1.2, we use them to characterize the cluster and the field in parallax and proper motion space by fitting a model that allows for intrinsic dispersions; and in Section 3.1.3, we classify the stars in different categories and calculate memberships probabilities, identifying highly likely cluster members.

\subsubsection{Gaia DR2 Data}

The astrometric data used in this work are from Data Release 2 (DR2) of the Gaia mission ${ }^{5}$ (Gaia Collaboration et al. 2018b). Gaia DR2 provides positions, proper motions, and parallaxes, as well as photometry in the $G, G_{\mathrm{BP}}$, and $G_{\mathrm{RP}}$ bands for $\sim 80 \%$ of its $\sim 1.7$ billion stars. Although Gaia also provides RV measurements for some of these stars, this subset only corresponds to $\lesssim 0.5 \%$ of the Gaia sample, and we therefore do not use this parameter in our membership study.

We download the Gaia DR2 data for all the sources contained within the region where the NGC 2547 rotation period catalog reports candidate cluster members (out to $\sim 50^{\prime}$ from the cluster center; see Section 2.1).

Regarding the Gaia DR2 parallaxes, as reported by Lindegren et al. (2018) and confirmed by several other works (Riess et al. 2018; Stassun \& Torres 2018; Schönrich et al. 2019; Zinn et al. 2019; Chan \& Bovy 2020), there is a zeropoint offset that needs to be considered. For the remainder of this paper, we adopt the global mean value of $29 \mu$ as for all the clusters (in the sense that the Gaia parallaxes are too small) reported by Lindegren et al. (2018), with the exception of NGC 6811 , for which we adopt the Kepler field $53 \mu$ as value from Zinn et al. (2019). Spatial variations in this zero-point offset are real but modest for the systems that we are interested in. Furthermore, since we are interested in studying stars at the same true distance, the exact zero-point value does not strongly impact our membership results (although it could impact our cluster ages; see Section 4).

\subsubsection{Cluster Fitting}

Our method is similar to membership probability studies found in the literature, and its goal is to separate the cluster population from the field population in phase space. For instance, Vasilevskis et al. (1958) and Sanders (1971) used a $2 \mathrm{D}$ version of this method using proper motions to compute memberships for a number of clusters. Other studies that have used similar versions of this method include Francic (1989), Jones \& Walker (1988), Jones \& Stauffer (1991), and Jones \& Prosser (1996). A 3D version including parallaxes as well as individual star measurement errors and correlations, in addition to allowing for intrinsic dispersions, has been recently used by Franciosini et al. (2018) and Roccatagliata et al. (2018, 2020).

In our method, a given star $i$ has a probability of belonging to either population $P$ (field $F$ or cluster $C$ ), with the total likelihood being:

$$
\mathcal{L}_{i}=f_{F} \mathcal{L}_{F, i}+f_{C} \mathcal{L}_{C, i}
$$

\footnotetext{
5 We note that Early Data Release 3 (EDR3) from Gaia became available (Gaia Collaboration et al. 2021) as this work was nearing completion; however, we do not anticipate that using these newer data would substantially change the results presented here. This arises from the fact we are studying predominantly nearby clusters, where the DR2 parallax and proper motion errors are already small. Additionally, EDR3 does not contain new RV data with respect to DR2.
}

where $f_{F}$ and $f_{C}$ represent the fraction of stars that belong to the field and cluster (normalized such that $f_{C}=1-f_{F}$ ), and $\mathcal{L}_{F, i}$ and $\mathcal{L}_{C, i}$ are the likelihoods of each population, respectively. We assume that the likelihood of each population $P$ can be described by a 3D multivariate Gaussian:

$$
\mathcal{L}_{P, i}=\frac{1}{(2 \pi)^{3 / 2}\left|\Sigma_{i}\right|^{1 / 2}} \exp \left[-\frac{1}{2}\left(\boldsymbol{x}_{i}-\boldsymbol{x}_{P}\right)^{T} \Sigma_{i}^{-1}\left(\boldsymbol{x}_{i}-\boldsymbol{x}_{P}\right)\right]
$$

where

$$
\boldsymbol{x}_{i}-\boldsymbol{x}_{P}=\left(\begin{array}{c}
\varpi_{i}-\varpi_{P} \\
\mu_{\alpha_{i}}-\mu_{\alpha_{P}} \\
\mu_{\delta_{i}}-\mu_{\delta_{P}}
\end{array}\right)
$$

and $\Sigma_{i}=C_{i}+\Sigma_{P}$, where $C_{i}$ is the individual covariance matrix, and $\Sigma_{P}$ is the matrix of intrinsic dispersions:

$$
\begin{aligned}
& C_{i}=\left(\begin{array}{ccc}
\sigma_{\varpi} & \rho_{\varpi \mu_{\alpha}, i} \sigma_{\varpi} \sigma_{\varpi_{i}} \sigma_{\mu_{\alpha_{i}}} & \rho_{\varpi \mu_{\delta}, i} \sigma_{\varpi} \sigma_{\varpi_{i}} \sigma_{\mu_{\delta_{i}}} \\
\rho_{\varpi \mu_{\alpha}, i} \sigma_{\varpi_{i}} \sigma_{\mu_{\alpha_{i}}} & \sigma_{\mu_{\alpha_{i}}}^{2} & \rho_{\mu_{\alpha} \mu_{\delta}, i} \sigma_{\mu_{\alpha_{i}}} \sigma_{\mu_{\delta_{i}}} \\
\rho_{\varpi \mu_{\delta}, i} \sigma_{\varpi_{i}} \sigma_{\mu_{\delta_{i}}} & \rho_{\mu_{\alpha} \mu_{\delta}, i} \sigma_{\mu_{\alpha_{i}}} \sigma_{\mu_{\delta_{i}}} & \sigma_{\mu_{\delta_{i}}}^{2}
\end{array}\right), \\
& \Sigma_{P}=\left(\begin{array}{ccc}
\sigma_{\varpi P}^{2} & 0 & 0 \\
0 & \sigma_{\mu_{\alpha P}}^{2} & 0 \\
0 & 0 & \sigma_{\mu_{\delta_{P}}}^{2}
\end{array}\right) .
\end{aligned}
$$

By using these equations on the Gaia data, we can derive the cluster and field parameters using a maximum likelihood fit.

To accurately derive these parameters, we apply a set of quality and geometric cuts. We highlight that these cuts are applied only when defining the subset of stars we use to derive the cluster and field astrometric parameters, but in Section 3.1.3 we apply our membership classification to the full Gaia sample (i.e., the stars excluded during this exercise are still classified later on). We run our maximum likelihood calculation using the subset of stars with astrometric_excess_noise $=0$ and apparent $G<18$ mag. In this way, we only fit the stars with well-defined astrometric solutions, and derive representative cluster parameters that are not affected by uncertain measurements of individual faint stars. We additionally note that the photometric monitoring survey that provides rotation period information searched for candidate members far in the outskirts of the cluster. In our cluster parameter calculation, however, we perform our fit using a subset of stars located closer to the cluster center (but not so close that the intrinsic dispersions we derive are affected by this decision).

We use Python's minimize function (method $=$ SLSQP) to run our maximum likelihood calculation and derive the cluster and field parameters (i.e., $\varpi_{C}, \mu_{\alpha_{C}}, \mu_{\delta_{C}}, \sigma_{\varpi_{C}}, \sigma_{\mu_{\alpha_{C}}}, \sigma_{\mu_{\delta_{C}}}$ for the cluster, and the analogous set of parameters for the field population). We report the calculated cluster parameters in Appendix B. All in all, although our algorithm to derive cluster parameters could have a higher degree of complexity (e.g., by calculating the parameters in a magnitude-dependent way), the membership classification presented in what follows is robust.

\subsubsection{Membership Probability Calculation}

Now that we have calculated the cluster and field parameters, we seek to evaluate Equation (1) for individual stars and 
calculate membership probabilities. First, however, the availability and quality of the Gaia astrometry need to be considered, as the data are not of equal precision for all stars, and they strongly depend on the target brightness. For instance, typical parallax (proper motion) uncertainties are of order

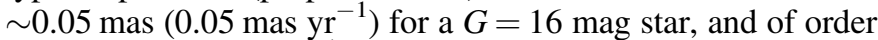
$\sim 0.5$ mas $\left(1.5 \mathrm{mas} \mathrm{yr}^{-1}\right.$ ) for a $G=20 \mathrm{mag}$ star.

Additionally, the presence of binary stars needs to be considered. Binary stars with measured periods are interesting targets for rotation studies (e.g., Stauffer et al. 2018; Tokovinin \& Briceño 2018), and can provide important clues when investigating rotation in stellar populations (e.g., Simonian et al. 2019, 2020). Because of this, and to not bias our rotational sequences, we purposely avoid discarding binary stars with our astrometric quality cuts.

We proceed as follows. From the full Gaia sample, we select the stars with available positions, proper motions, and parallax values. Additionally, following Gaia Collaboration et al. (2018a), we select stars with visibility_periods_ used $>8$ and with $\sqrt{\chi^{2} /\left(\nu^{\prime}-5\right)}<1.2 \max (1, \exp (-0.2(G-$ 19.5))), where $\chi^{2} \equiv$ astrometric_chi2_al and $\nu^{\prime} \equiv$ astrometric_n_good_obs_al. The latter of these quality cuts removes most of the artifacts while retaining genuine binaries (where naturally the single-star solution does not provide a perfect fit; e.g., see Belokurov et al. 2020).

The stars excluded by these quality cuts do not have enough information for a reliable membership probability to be calculated. Nonetheless, we do not remove them from our sample, as some of them could correspond to stars with measured rotation periods. We assign them the classification flag of no info stars and include them in the NGC 2547 rotational sequence shown in Section 5.

The stars that survive these astrometric quality cuts have enough information such that we can perform a thorough membership classification. At this point, we separate stars that could be cluster members from those that are most likely not members. For a given star $i$, we calculate the quantity:

$$
\Delta_{i}=\frac{1}{\sqrt{3}} \sqrt{\frac{\left(\varpi_{C}-\varpi_{i}\right)^{2}}{\left(\sigma_{\varpi_{C}}^{2}+\sigma_{\varpi_{i}}^{2}\right)}+\frac{\left(\mu_{\alpha_{C}}-\mu_{\alpha_{i}}\right)^{2}}{\left(\sigma_{\mu_{\alpha_{C}}}^{2}+\sigma_{\mu_{\alpha_{i}}}^{2}\right)}+\frac{\left(\mu_{\delta_{C}}-\mu_{\delta_{i}}\right)^{2}}{\left(\sigma_{\mu_{\delta_{C}}}^{2}+\sigma_{\mu_{\delta_{i}}}^{2}\right)}},
$$

where values with the $C$ index represent the cluster parameters derived in Section 3.1.2. We classify the stars with values of $\Delta_{i}>3$ as non members, as they are located outside the cluster's $3 \sigma$ ellipsoid in phase space.

All of the remaining unclassified stars have $\Delta_{i} \leqslant 3$ and are technically consistent with the cluster phase-space parameters (at the $3 \sigma$ level). This population, however, has a contribution from objects that have large astrometric uncertainties (particularly in parallax), for which a reliable classification is hard to determine with the existing data. Therefore, another selection criterion is required in order to separate the likely cluster members from this ambiguous population. For this purpose, we again use the method described in Section 3.1.2, and calculate membership probabilities for all objects with $\Delta_{i} \leqslant 3$. Following Equation (1), for a given star $i$, the cluster membership probability is calculated as

$$
P_{C, i}=\frac{f_{C} \mathcal{L}_{C, i}}{\mathcal{L}_{i}}
$$

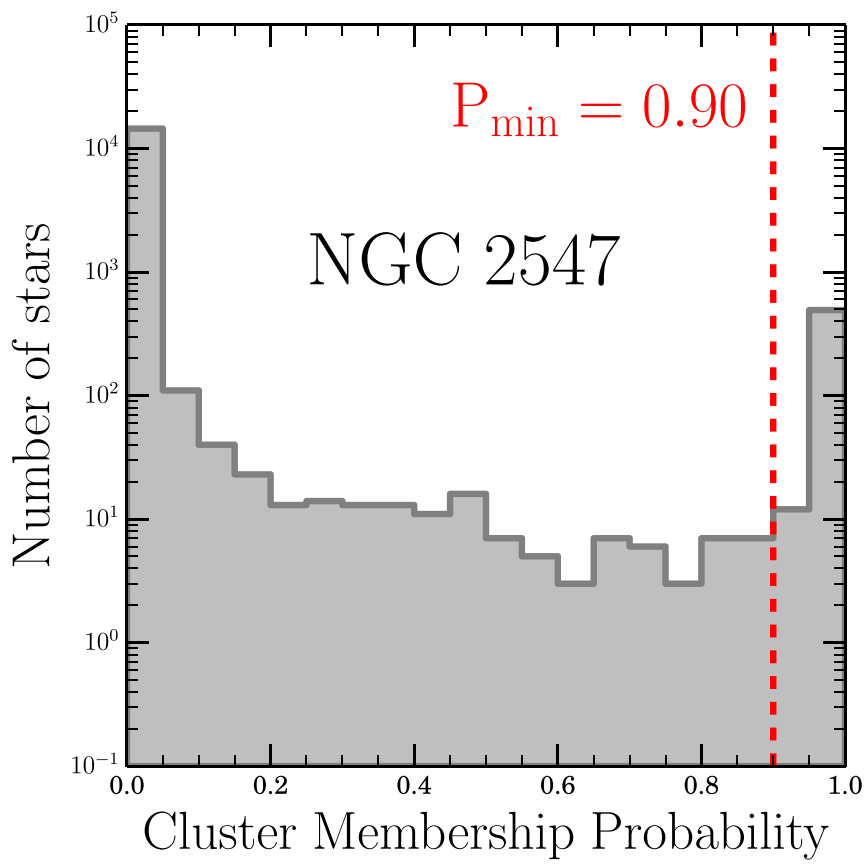

Figure 1. NGC 2547: membership probability distribution for the stars with $\Delta_{i} \leqslant 3$ (i.e., stars that could be cluster members, as they are inside the cluster's $3 \sigma$ ellipsoid in phase space). The peak at probabilities close to 1 represents the population of likely cluster members. The vertical red dashed line, $P_{\min }=0.90$, shows the probability threshold we use to separate the probable members $\left(P_{C} \geqslant P_{\min }\right)$ from the possible members $\left(P_{C}<P_{\min }\right)$.

Conversely, this corresponds to $1-P_{F, i}$, where $P_{F, i}$ is the field membership probability for the star $i$. The distribution of cluster membership probabilities for all stars with $\Delta_{i} \leqslant 3$ in the NGC 2547 field is shown in Figure 1.

For NGC 2547, we find the distribution of cluster membership probabilities to be strongly bimodal, with most of the sample having probabilities close to either 0 or 1 . Although not explicitly shown, the membership probability distribution has a strong dependence on the stars' apparent $G$-band magnitude, with the distribution being mostly bimodal for bright stars and becoming blurrier for fainter stars (with increasingly more stars having intermediate probability values; e.g., see Figure 5 of Jones \& Prosser 1996). This magnitude-dependent outcome is typical, as bright stars tend to have more precise astrometric measurements, and the method provides a yes or no answer regarding their memberships. On the other hand, faint stars typically have larger astrometric uncertainties, which only allow the method to provide an intermediate answer.

With this in mind, we apply a final selection criterion and classify the stars with $P_{C} \geqslant 0.90$ as probable members and those with $P_{C}<0.90$ as possible members. We show the resulting phase-space projections and apparent CMD of these populations, in addition to the non member population, in Figures 2 and 3. (The no info population is absent from these figures, as no astrometric information is available for those stars.)

We choose the probability threshold of $P_{\min }=0.90$ to separate between probable and possible members as a compromise between completeness in our sample of probable cluster members on the one hand, and the presence of contamination on the other. Several tests with different $P_{\min }$ values showed that the phase space and CMD of the probable members seemed to lose bona fide cluster stars when using 

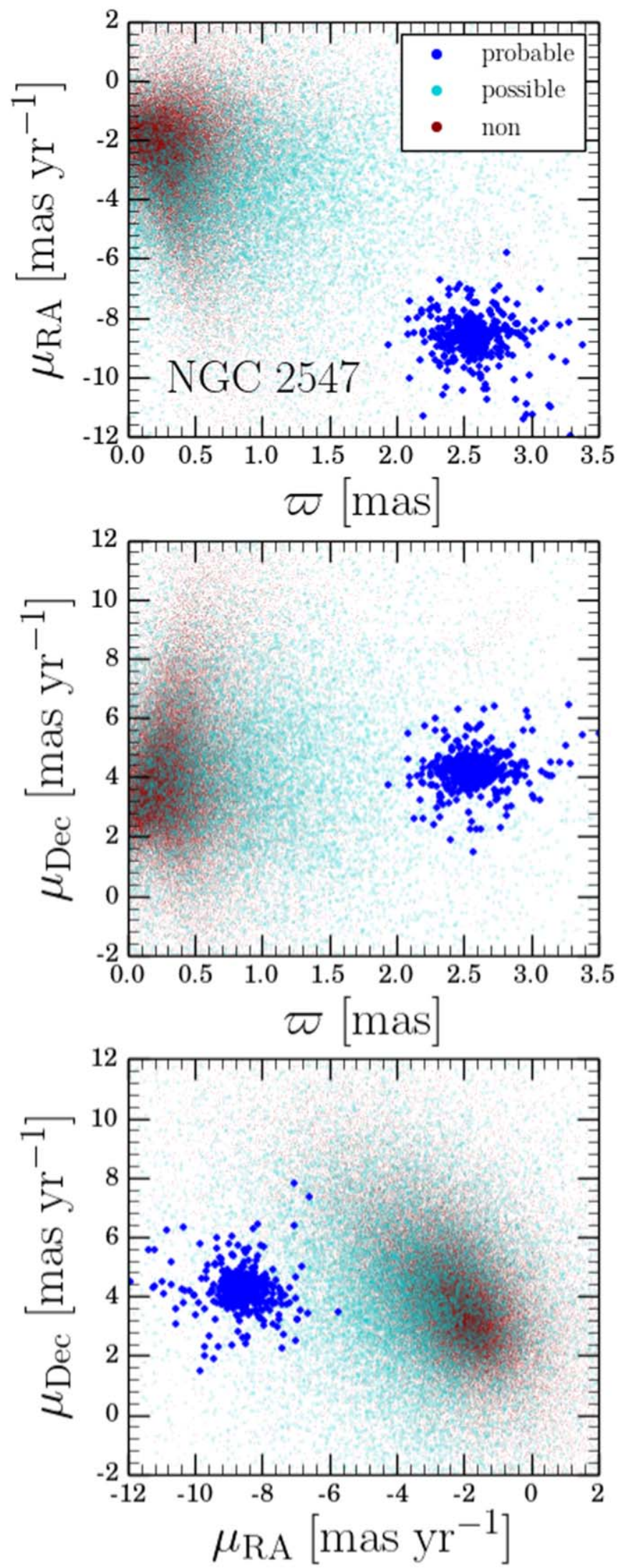

Figure 2. NGC 2547: phase-space projections of the probable (blue), possible (cyan), and non (red) members defined in Section 3.1.3. The probable members are clustered in phase-space, while the non members (i.e., field stars) populate a much larger region at smaller parallax and proper motion values. The possible members correspond to an intermediate population, dominated by faint stars with large astrometric uncertainties.

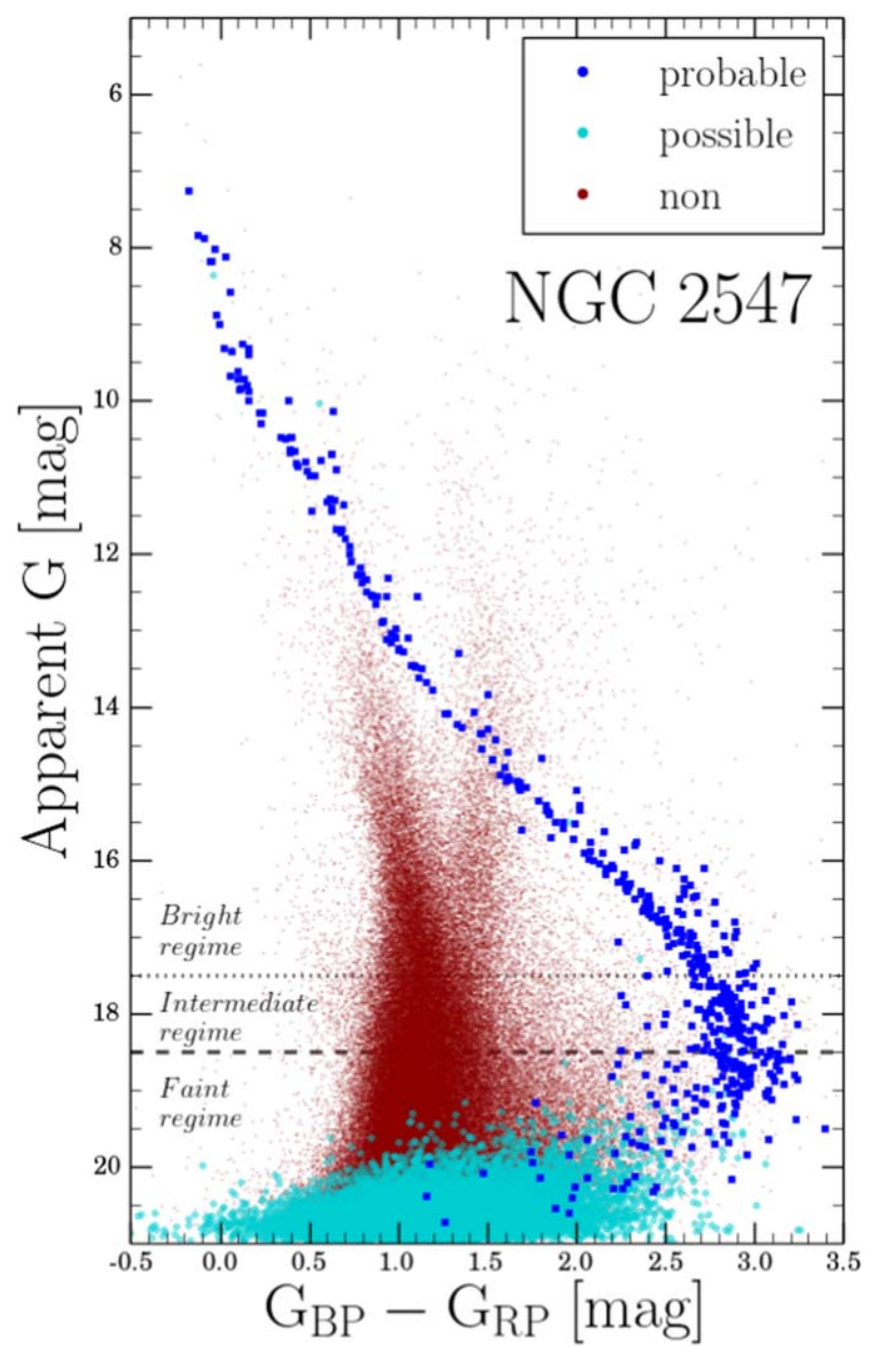

Figure 3. NGC 2547: apparent $G$-band vs. $G_{\mathrm{BP}}-G_{\mathrm{RP}} \mathrm{CMD}$ of probable (blue), possible (cyan), and non (red) members defined in Section 3.1.3. Although no photometric selection criteria was used to define the three populations, the probable members form an isochrone-like sequence, confirming that our selection method is identifying likely cluster members. The non members show the typical behavior of the background field, while the possible members mainly consist of faint stars $(G \gtrsim 19.5 \mathrm{mag})$. The turnover to bluer colors for apparent $G \gtrsim 18.5 \mathrm{mag}$ for the probable members sequence is discussed in Section 3.1.3. The dotted and dashed lines show the $G=17.5$ and 18.5 mag limits, which we use to define different regimes in our mass and temperature calculation (see Section 4.2).

higher $P_{\min }$ values, while lower values included stars with incoherent kinematics and photometry. We nonetheless note that the overall number of probable and possible members do not considerably change unless very high $\left(P_{\min } \gtrsim 0.95\right)$ or very low $\left(P_{\min } \lesssim 0.05\right)$ threshold values are used (see Figure 1$)$. Furthermore, Appendix $\mathrm{C}$ reports the Gaia data for the probable and possible members and their cluster membership probabilities, and the reader is free to experiment with customized threshold values.

Figure 2 shows that the probable members (blue points) tend to be clustered in phase space with a small number of stars located in the outskirts. On the other hand, the non members (i.e., the field; red points) occupy a much larger region in the phase-space projections (with stars populating virtually all corners of the diagrams), but tend to be concentrated at smaller parallax and proper motion values. The possible members 
(cyan points) appear as an intermediate population of stars, located in between the cluster and the field.

Figure 3 complements what is seen in Figure 2. The probable members form a tight isochrone-like sequence in the apparent CMD (with the exception of the color turnover for apparent $G \gtrsim 18.5 \mathrm{mag}$; see discussion below), with even a parallel sequence being visible indicating the presence of photometric binaries (e.g., Hurley \& Tout 1998). The non members behave as expected from the field, with the two main branches (main-sequence and giant branch) being clearly visible in the data. The possible members mainly populate the bottom part of the plot, demonstrating that this population is dominated by faint stars that, consequently, have large astrometric uncertainties, which in turn causes them to have $\Delta_{i} \leqslant 3$ values (differentiating them from the non members). While some of these possible members could very well be real cluster members, the current precision of their astrometric information does not allow for a more stringent classification.

We highlight that, even though our classification method is completely agnostic to the photometry of the stars, and it is solely based on kinematics, it seems to appropriately select stars that form a main sequence in the CMD. We take this as a confirmation that our method is properly identifying likely cluster members and distinguishing them from the field.

A peculiar feature can be seen in Figure 3 for apparent $G \gtrsim 18.5$ mag. At this apparent magnitude, instead of continuing to the bottom right part of the diagram, the probable members start to turn to bluer colors for fainter magnitudes. This feature has already been found by other works when using the Gaia DR2 photometry (e.g., Arenou et al. 2018; Lodieu et al. 2019a, 2019b; Smart et al. 2019), and it arises from an overestimation of the flux in the $G_{\mathrm{BP}}$ passband for faint, red sources (Riello et al. 2018, 2021).

\subsection{Crossmatch}

Now that we have kinematically classified every Gaia DR2 star in the field of NGC 2547 as either a probable, possible, or non member, or else a no info star, we proceed to connect this with the rotation sample of Irwin et al. (2008).

We crossmatch both catalogs using the CDS X-Match Service on VizieR. ${ }^{6}$ First, we download all the Gaia DR2 matches to a given NGC 2547 star within $3^{\prime \prime}$. This produces matches for all stars, with some of them $(\sim 10 \%)$ being matched to two Gaia sources. The distribution of angular separations is strongly peaked at small values, with $\sim 80 \%$ of the stars having a match within 0 .' 2 , a tail extending out to 0 ". 5 , and some matches extending past this value out to $3^{\prime \prime}$.

To select the best possible matches for stars matched to two Gaia sources, we compare the $V$ magnitudes from Irwin et al. (2008) with the $G$ magnitudes from Gaia. This exercise helps us break the ties and decide which star is the correct match. In all cases, the Gaia star with the more similar brightness corresponds to the closer match in angular separation. The second matched star is typically several magnitudes fainter, with angular separations greater than $1^{\prime \prime}$ (the outliers of the separation distribution). We only keep one Gaia match per star, and are left with an angular separation distribution where $\sim 95 \%$ of the stars have a match within $0 . " 3$ and all of them have a match within $0 . \prime 9$.

\footnotetext{
6 http://vizier.u-strasbg.fr/
}

In order to test the reliability of our crossmatching approach, we use the Pleiades cluster as a benchmark for which a completely independent crossmatching is available. We first replicate the same crossmatching procedure used in NGC 2547 with our Pleiades data. We then take the 2MASS IDs for the Pleiades stars from Rebull et al. (2016a) and look them up in the precomputed 2MASS-Gaia DR2 crossmatch (gaiadr2. tmass_best_neighbor) that can be found in the ESA's webpage of the Gaia Archive. ${ }^{7}$ We find an excellent agreement ( $>99.7 \%$ of coincidence) between ESA's crossmatch and ours for the stars found by both methods (and moreover, VizieR produces matches for 10 Pleiades stars not found by ESA). We take this as a confirmation that our crossmatching is properly combining the periodic samples with the Gaia sources.

\subsection{Applying Our Method to All the Clusters}

We follow the method described in Section 3.1 for NGC 2547, and apply it to the other six clusters listed in Table 1 . We report the astrometric parameters we calculate for the clusters in Appendix B, where we also compare them with the values reported in the literature. We find our astrometric parameters to be in good agreement with those reported by other studies, with fractional differences being typically at the $\sim 1 \%-2 \%$ level or less.

For each cluster, we classify every Gaia star contained within the region where the corresponding rotation period catalog reports candidate members into one of the four previously described categories (no info, probable, possible, or non member). We report tables with the corresponding list of probable and possible members for every cluster in Appendix C. Analogously to Figures 1 and 3 for NGC 2547, Figures 4 and 5 show the membership probability distribution and apparent CMDs for all the clusters we study. The phasespace projections for all clusters, analogous to Figure 2 for NGC 2547, can be found in Appendix D.

Figure 4 shows that, for every cluster, in the sample of Gaia stars that satisfy $\Delta_{i} \leqslant 3$, there is a population of stars with membership probabilities close to 1 . This population represents a set of likely cluster members, and we classify those with $P_{C} \geqslant 0.90$ as probable members (and the rest as possible members). On the low-probability end, however, clear clusterby-cluster differences can be observed. While clusters such as the Pleiades and Praesepe show only a small number of possible members, M50 and NGC 6811 are in the opposite situation. These differences are explained by considering how similar or different the astrometric parameters of a given cluster are, compared with the field (which is typically a diffuse background population with small parallax and low proper motion). In other words, a cluster with a large parallax and proper motion (e.g., the Pleiades or Praesepe) will be more distinct and separated from the field in phase space, and therefore it is highly unlikely that unassociated field stars will have similar astrometry (i.e., $\Delta_{i} \leqslant 3$ ), and hence a small number of possible members is expected. On the other hand, field stars are more likely to mimic the astrometry of a cluster that has a small parallax and a low proper motion (e.g., M50 or NGC 6811), and therefore the number of stars that are kinematically consistent with the cluster increases.

The apparent CMDs of all our clusters, shown in Figure 5, display an isochrone-like sequence of probable members, with

\footnotetext{
http://gea.esac.esa.int/archive/
} 


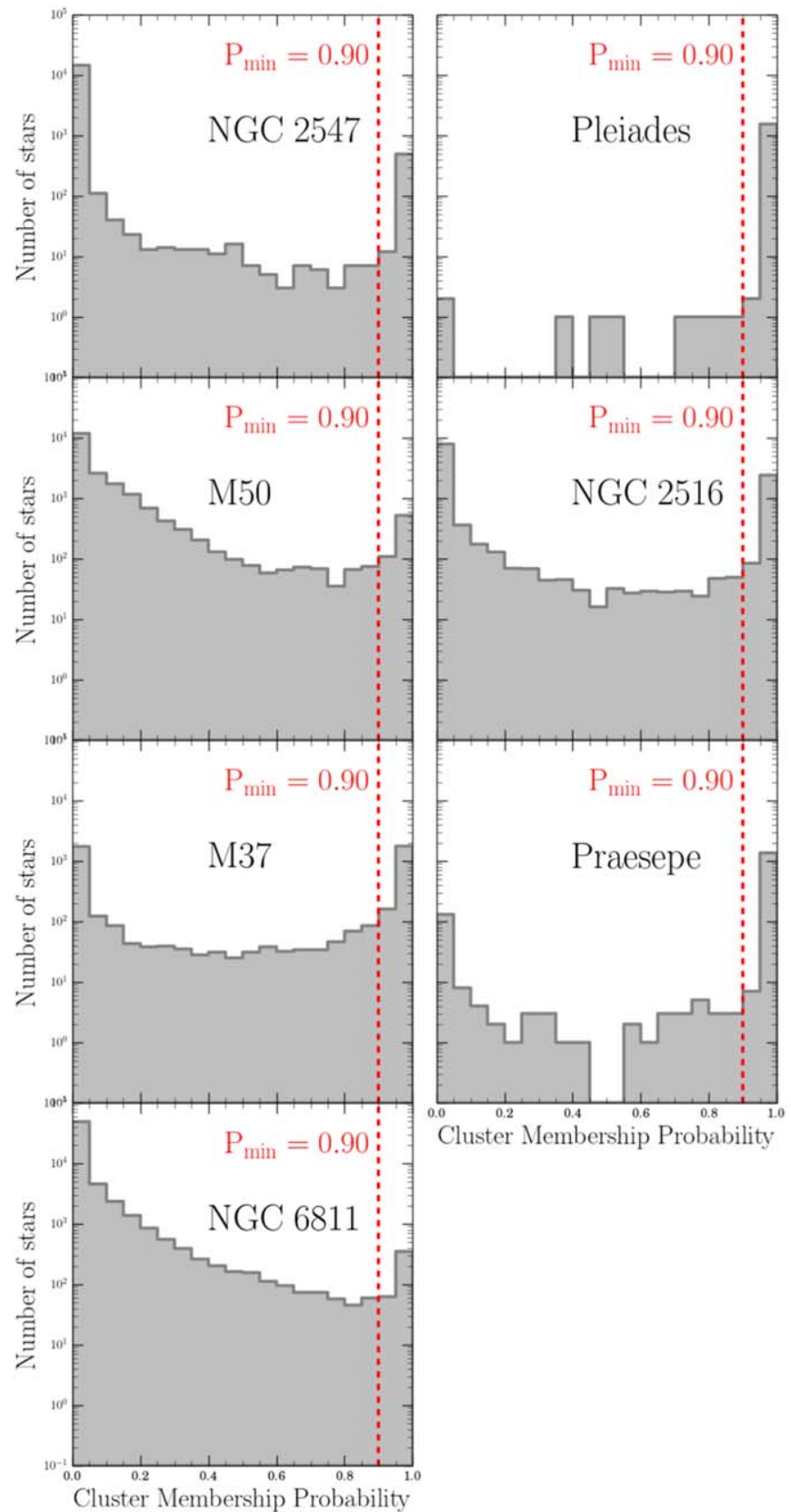

Figure 4. Membership probability distribution for all the clusters we study, analogous to Figure 1.

clear binary sequences being identified in all of them. The older clusters M37, Praesepe, and NGC 6811 show not only a mainsequence population but also a few giant stars (with Praesepe even showing a clear white dwarf sequence). We highlight that, in order to obtain unbiased cluster memberships, our method is completely agnostic to the Gaia photometry, and these results are a consequence of our careful astrometric analysis. Additionally, as previously found for NGC 2547, we observe the turnover to bluer $G_{\mathrm{BP}}-G_{\mathrm{RP}}$ colors at apparent magnitudes $G \gtrsim 18.5 \mathrm{mag}$ in the probable member sequence of all the clusters. We take this as further evidence that this corresponds to an artifact of the Gaia photometry (see Section 3.1.3 for details).

As discussed in Section 3.1.3, our ability to cleanly separate between possible and probable members is a strong function of

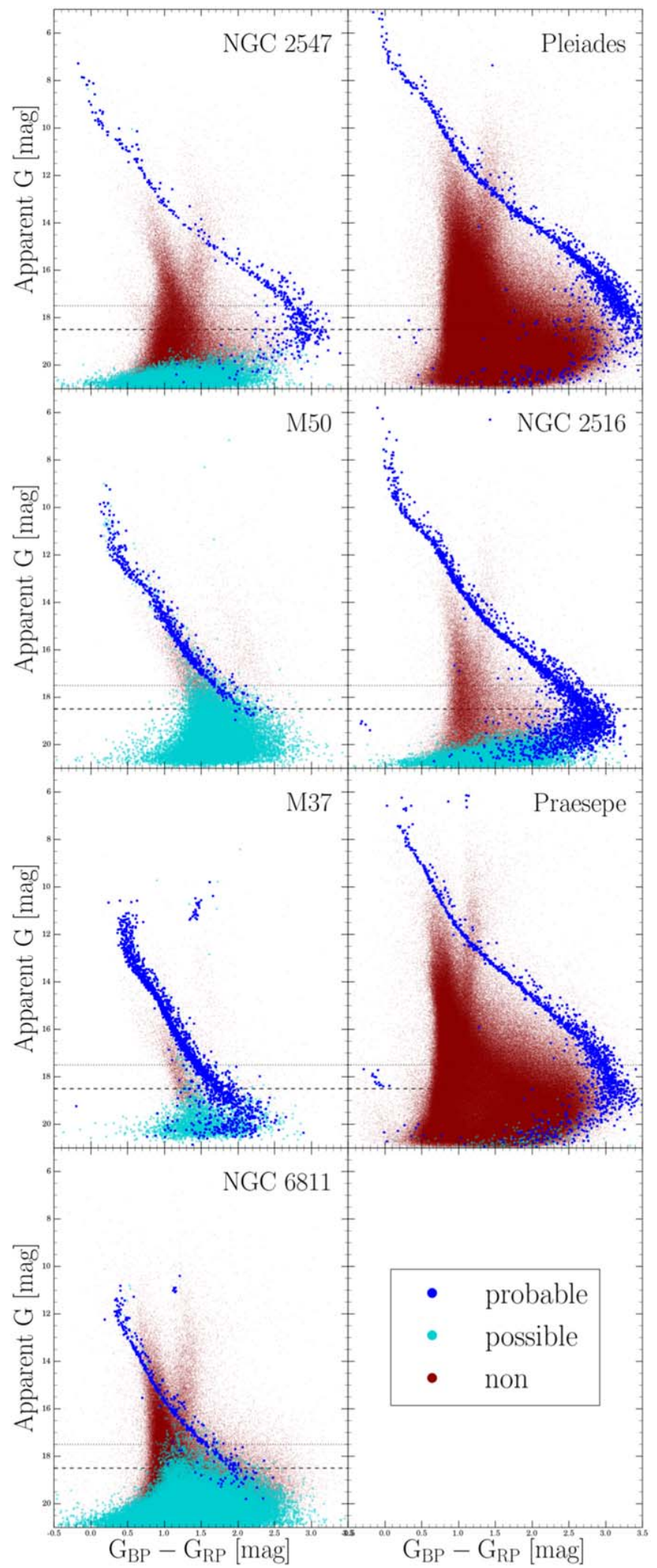

Figure 5. Apparent $G$-band vs. $G_{\mathrm{BP}}-G_{\mathrm{RP}} \mathrm{CMD}$ of probable (blue), possible (cyan), and non (red) members for all the clusters we study, analogous to Figure 3.

the stars' apparent $G$-band magnitude, as the quality of their astrometry decreases with decreasing brightness. This can be clearly seen, for instance, in the CMD of NGC 6811, where all the stars fainter than apparent $G \simeq 19$ mag are classified as 
either possible or non members, and none of them are classified as probable members. Real NGC 6811 members with $G>19$ mag are simply too faint for Gaia DR2 to provide precise astrometry, preventing us from reliably differentiating them from the field stars.

The crossmatching between the periodic samples and the Gaia DR2 data is done in identical fashion for all of the clusters, following the approach of Section 3.2. Unlike the case of NGC 2547, however, we do not find a Gaia DR2 counterpart for every periodic star in most of the other clusters. Our recovery rates (and number of stars not found in the crossmatching over the number of stars with measured periods) are: $100 \%$ (0/176) for NGC 2547, 99.9\% (1/759) for Pleiades, $94.3 \%(46 / 812)$ for M50, 99.4\% (2/364) for NGC 2516 , $96.7 \%$ (12/367) for M37, 99.8\% (2/809) for Praesepe, and $100 \%(0 / 232)$ for NGC 6811 . The only clusters with recovery rates below $99 \%$ are M50 and M37, which correspond to distant systems where the periodic samples extend to magnitudes fainter than the apparent $G \approx 21 \mathrm{mag}$ limit of Gaia DR2. In the following, we classify the stars not found in the crossmatching as no info stars, as their membership is uncertain and we do not have evidence to discard them.

\section{Cluster and Stellar Properties}

\subsection{Cluster Properties}

To perform meaningful intercluster comparisons of stellar rotation, reliable cluster properties are needed. In the pre-Gaia era, classic works such as Denissenkov et al. (2010), Gallet \& Bouvier (2013), and Gallet \& Bouvier (2015) thoroughly compiled periodic samples as well as properties for a large number of clusters. Nevertheless, the heterogeneous nature of their parameter compilation (adopting values from varied techniques and data sets) can result in inhomogeneities in the adopted masses, ages, and metallicity scales. Nowadays, the systematic observations of star clusters by spectroscopic surveys (Netopil et al. 2016; Gaia-ESO, Magrini et al. 2017; Spina et al. 2017; APOGEE, Majewski et al. 2017; Jönsson et al. 2020), as well as the uniform all-sky Gaia data, provide an opportunity to study these systems in a more consistent and homogeneous fashion. In the following, we attempt, to the extent that it is possible, to compile and derive cluster properties that are in a consistent and uniform scale.

The mass and temperature values we calculate in Section 4.2, as well as the results and discussion presented in Sections 5 and 6 , require four cluster properties to be known: distance modulus, reddening, age, and metallicity. The former two are needed to translate the observed photometry to absolute and dereddened CMD space, while the latter two are required to compare the data with the appropriate stellar evolutionary model. For distance modulus, we adopt the values derived from our astrometric analysis (considering the parallax zero points described in Section 3.1.1), and for metallicity, we compile values reported from recent high-resolution spectroscopic results. In particular, we adopt the state-of-the-art values reported by Netopil et al. (2016) for Pleiades, NGC 2516, M37, Praesepe, and NGC 6811, and the value reported by the GaiaESO survey (Spina et al. 2017) for NGC 2547. The only cluster without a spectroscopic metallicity measurement is M50, for which we adopt a solar value. We now describe our procedure to obtain cluster reddenings and ages.
Instead of adopting reddening values from the literature, we calculate our own using a dedicated fitting routine. For a given cluster, we start by correcting the observed $G$-band magnitudes by distance modulus. Once the spectroscopic metallicity is known, we download a set of PARSEC models ${ }^{8}$ (Bressan et al. 2012; Chen et al. 2014) of that composition with a range of plausible ages (e.g., 100, 200, and $300 \mathrm{Myr}$ for NGC 2516). Then, we define a region in the absolute CMD where the different models agree with each other, which excludes the phases near and more evolved than the turnoff. We then iterate over a range of possible reddenings and find the value that produces the best agreement between the observed data with the series of models.

For a given $E(B-V)$ value, we assume $A_{V}=3.1 \times E(B-V)$ (Cardelli et al. 1989) and deredden the photometry as follows. For stars with measured photometry in the three Gaia bands, we deredden the observed magnitudes following the approach of Gaia Collaboration et al. (2018a), where the extinction coefficients depend on $G_{\mathrm{BP}}-G_{\mathrm{RP}}$ color and the extinction itself. For stars that lack a measured $G_{\mathrm{BP}}-G_{\mathrm{RP}}$ color, we adopt the PARSEC coefficients ${ }^{9}$ for a G2V star $\left(A_{G} / A_{V}=0.85926\right.$, $A_{G_{\mathrm{BP}}} / A_{V}=1.06794$, and $\left.A_{G_{\mathrm{RP}}} / A_{V}=0.65199\right)$.

For every $E(B-V)$ value we are iterating over, we deredden the probable cluster members' photometry, define a set of bins in the $\left(G_{\mathrm{BP}}-G_{\mathrm{RP}}\right)_{0}$ color coordinate, and calculate the mean colors and 75th magnitude percentiles (which accounts for the photometric binaries). We then find the $E(B-V)$ value that minimizes the sum of the squared difference of the data minus the models given the bins, and adopt this as our global cluster reddening. Finally, we compare the CMD location of the full sample of probable members with models of varying ages, and estimate the age following the envelope of hot stars below and near the turnoff (typically stars hotter than $\sim 7000 \mathrm{~K}$ ). We estimate that this procedure leads to typical errors of $\sim 20 \%$ for reddenings and $\sim 10 \%-20 \%$ for ages.

We summarize the revised cluster properties we adopt in Table 2. Note that, for the nearby and young clusters NGC 2547 and the Pleiades, we adopt the lithium depletion ages from Jeffries \& Oliveira (2005) (see also Naylor \& Jeffries 2006) and Stauffer et al. (1998), respectively. This technique yields reliable and almost completely modelindependent ages in the 10-200 Myr range. These adopted ages are in good agreement with the values we derive from our CMD analysis.

\subsubsection{Comparing Our Cluster Properties with the Literature}

We now compare the cluster properties we have derived versus the values that can be found in the literature from similar approaches. The main references we use for this are Gaia Collaboration et al. (2018a) and Bossini et al. (2019), who also studied open clusters based on the Gaia DR2 data, and reported parameters for most of the clusters in our sample based on comparisons with PARSEC models. We also use as reference the works by Cummings et al. $(2016,2018)$ and Cummings \& Kalirai (2018). Although these did not use Gaia data, they

\footnotetext{
8 We initially also considered using the MIST (Choi et al. 2016; Dotter 2016) and BHAC15 (Baraffe et al. 2015) models, but the PARSEC models showed better agreement when compared with the data. This is not surprising, as the PARSEC models include empirical corrections to fit the mass-radius relation of dwarf stars, which in turn produces a better agreement with the CMDs of clusters.

9 http://stev.oapd.inaf.it/cgi-bin/cmd_3.3
} 
Table 2

Revised and Updated Cluster Properties

\begin{tabular}{lccccc}
\hline \hline Name & $\begin{array}{c}\text { Age } \\
(\mathrm{Myr})\end{array}$ & $\begin{array}{c}{[\mathrm{Fe} / \mathrm{H}]} \\
(\mathrm{dex})\end{array}$ & $\begin{array}{c}\mathrm{DM} \\
(\mathrm{mag})\end{array}$ & $\begin{array}{c}\text { Distance } \\
(\mathrm{pc})\end{array}$ & $\begin{array}{c}E(B-V) \\
(\mathrm{mag})\end{array}$ \\
\hline NGC 2547 & 35 & -0.01 & 7.925 & 384.6 & 0.044 \\
Pleiades & 125 & -0.01 & 5.670 & 136.2 & 0.051 \\
M50 & 150 & 0.00 & 9.936 & 970.9 & 0.210 \\
NGC 2516 & 150 & +0.05 & 8.058 & 408.8 & 0.103 \\
M37 & 500 & +0.02 & 10.787 & 1436.8 & 0.246 \\
Praesepe & 700 & +0.16 & 6.345 & 185.8 & 0.014 \\
NGC 6811 & 950 & +0.03 & 10.167 & 1080.1 & 0.047 \\
\hline
\end{tabular}

Note. Summary of the revised and updated cluster properties (age, metallicity, distance modulus, and reddening) that we adopt to calculate masses and temperatures, as well as in our discussion throughout Section 6. The cluster distances are also shown for reference. We calculate distance moduli from our astrometric analysis, survey the literature for updated high-resolution spectroscopic metallicity measurements, and estimate reddening and age from a CMD analysis of the probable cluster members.

reported parameters for many of the clusters in our sample based on CMD fits to UBV photometry and PARSEC models.

For distance modulus, our values are calculated from the global cluster parallaxes, accounting for a zero-point value of $53 \mu$ as in NGC 6811 and of $29 \mu$ as for the other clusters. While Gaia Collaboration et al. (2018a) and Bossini et al. (2019) (whose membership and astrometry come from Cantat-Gaudin et al. 2018) do not consider these zero points, in Appendix B we add these offsets to their parallax values for an appropriate comparison and find differences to be within $\approx \pm 1 \%$. For metallicity, we share references and use high-resolution spectroscopic values from Netopil et al. (2016) or Gaia-ESO when available.

For reddening, if we exclude M50 from the comparison, we find good agreement with Gaia Collaboration et al. (2018a) and Bossini et al. (2019), and the differences between our values and theirs are contained within $\pm 0.032 \mathrm{mag}$ in $E(B-V)$. On the other hand, M50 is the only cluster for which the Gaia Collaboration et al. (2018a) and Bossini et al. (2019) reddenings differ between each other. The former reports $E(B-V)=0.105 \mathrm{mag}$, the latter reports $0.153 \mathrm{mag}$, and we obtain a best-fit value of $0.210 \mathrm{mag}$. We note, however, that Cummings et al. (2016) and Cummings \& Kalirai (2018) report $E(B-V)=0.230$ for $\mathrm{M} 50$, in better agreement with our estimate. Ultimately, the above differences in reddening are perhaps unsurprising, considering the different CMD analysis approaches and parallax zero-point considerations.

Regarding stellar ages, we find our revised values to be in good agreement with the pre-Gaia values compiled by Gallet \& Bouvier (2015) listed in Table 1 (see also Denissenkov et al. 2010; Gallet \& Bouvier 2013), especially considering the associated uncertainties. The largest difference is seen for Praesepe, for which we derive an age of $700 \mathrm{Myr}$, while Gallet \& Bouvier (2015) adopt 580 Myr. Given the crucial importance that clusters' ages play in models of angular momentum evolution, the overall good agreement between our values and the pre-Gaia ones is a meaningful result of our work.

Comparing our ages with those found by Gaia Collaboration et al. (2018a), we find our results to be in good agreement. A similar comparison with Bossini et al. (2019) shows that their ages seem to be systematically underestimated by factors of $30 \%-60 \%$ for the younger clusters. The exception to the above is NGC 2516, for which Gaia Collaboration et al. (2018a) and
Bossini et al. (2019) report ages of $\approx 300$ and $250 \mathrm{Myr}$, respectively. If confirmed, this would position NGC 2516 as an important anchor for stellar rotation at intermediate ages, as few systems older than the Pleiades and younger than M37 have such comprehensive periodic data sets. Nevertheless, our analysis favors the classical $\sim 150 \mathrm{Myr}$ age, and this value is similar to the results by Cummings et al. $(2016,2018)$ and Cummings \& Kalirai (2018) (see also Fritzewski et al. 2020; Healy \& McCullough 2020, and Bouma et al. 2021).

\subsection{Stellar Properties}

In order to perform comprehensive comparisons of the rotational sequences of the open clusters, we need to place them on a common scale. For this, we derive mass and temperature values for the cluster members by comparing the photometric data with state-of-the-art evolutionary models in absolute magnitude space. Although the papers that reported periods also reported photometry in at least two bands for most of our clusters (e.g., $V$ and $I$ for NGC 2547; see Section 2), the specific filters vary on a cluster-by-cluster basis. Instead of using these, we take advantage of the uniform $G, G_{\mathrm{BP}}$, and $G_{\mathrm{RP}}$ photometry provided by Gaia DR2. This also allows us to derive masses and temperatures for all the Gaia cluster members, not just the subset with measured periods.

We again illustrate our procedure using NGC 2547 as a working example. We use its $E(B-V)$ and distance modulus values (see Section 4.1) and calculate absolute and dereddened photometry. We show the extinction-corrected absolute CMD of the probable and possible NGC 2547 members in Figure 6. The data can now be directly compared with stellar models to infer mass and temperature values. We use a PARSEC isochrone with the corresponding NGC 2547 age and metallicity, and show it as the orange line in Figure 6.

The bright probable cluster members show an excellent agreement with the model in Figure 6. As discussed in Figure 3 , the $G_{\mathrm{BP}}-G_{\mathrm{RP}}$ color misbehaves for apparent $G \gtrsim 18.5 \mathrm{mag}$, and the probable member sequence becomes bluer for fainter magnitudes. This limit is translated to the absolute and dereddened CMD and shown as the horizontal dashed line in Figure 6, with the dotted line showing a similar apparent $G=17.5 \mathrm{mag}$ limit. We use these two apparent magnitude limits, here translated to absolute magnitude space, to define different regimes in our mass and temperature calculation.

Additionally, whenever possible, the effects of binarity on the CMD need to be considered. Photometric binaries appear as brighter/redder stars compared to the main-sequence locus, and to account for them we follow the procedures of Stauffer et al. (2016) and Somers et al. (2017). In this approach, when the Gaia $\left(G_{\mathrm{BP}}-G_{\mathrm{RP}}\right)_{0}$ color is reliable, stars are projected down (or up) onto the main-sequence locus at fixed color, effectively removing the contribution of close companions (i.e., our mass and temperature estimates for these stars correspond to those of the primary star). We designate the projected absolute magnitudes as $M_{G_{0, \text { proj }}}$, and describe its calculation in detail below.

To acknowledge the misbehavior of the $G_{\mathrm{BP}}-G_{\mathrm{RP}}$ color and account for the contribution of photometric binaries when possible, we define three regimes to calculate projected magnitudes $M_{G_{0, \text { proj }}}$ in the absolute CMD of Figure 6: 


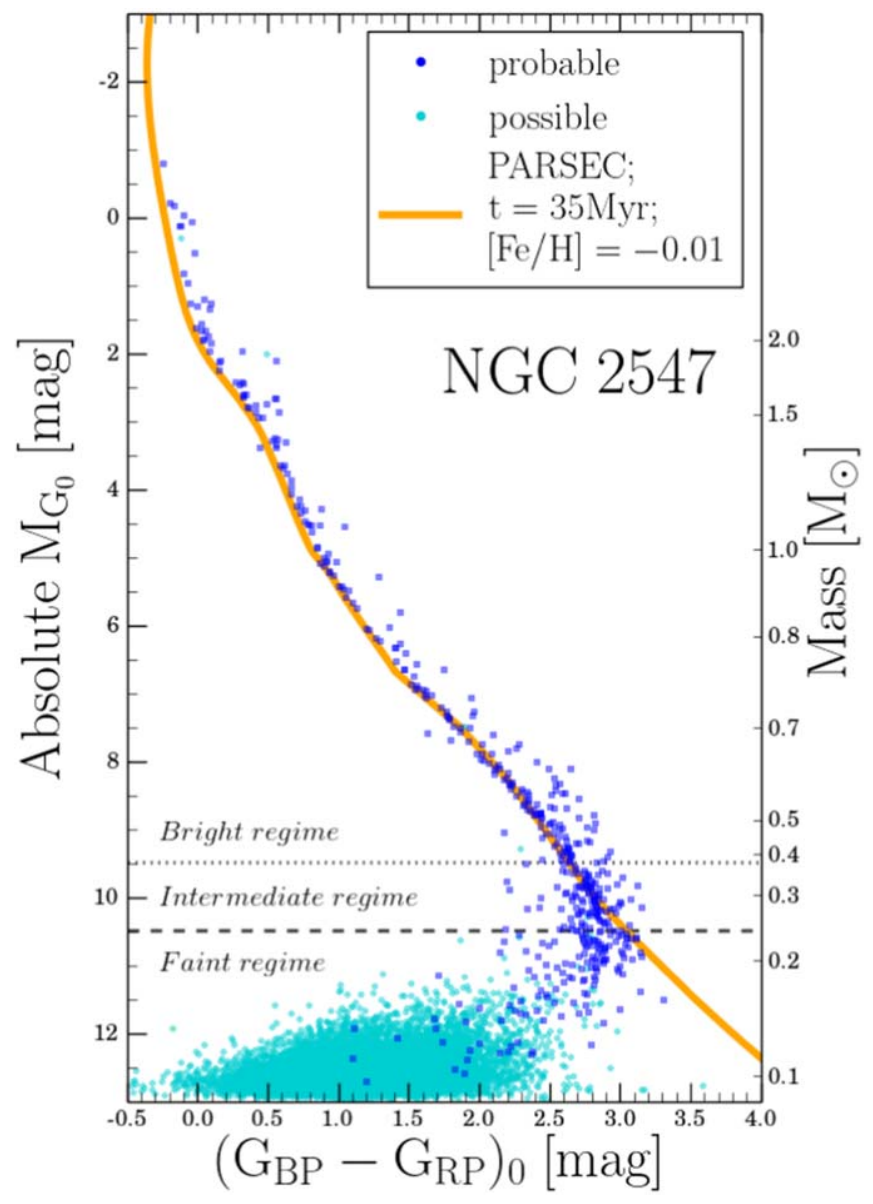

Figure 6. NGC 2547: absolute and dereddened CMD of the probable (blue) and possible (cyan) members. The points from Figure 3 have been corrected by extinction and distance modulus. The best-fit NGC 2547 PARSEC isochrone is shown as the orange line. The dotted and dashed lines correspond to the apparent $G=17.5$ and 18.5 mag limits from Figure 3 translated to absolute CMD space, which define different regimes in our calculation. We use this figure to interpolate the model and derive mass and temperature values (see Section 4.2).

1. Bright regime: For stars brighter than the dotted line (apparent $G=17.5 \mathrm{mag}$ translated to absolute magnitude), we calculate $M_{G_{0, \text { proj }}}$ by projecting the stars $M_{G_{0}}$ value down (or up) onto the model at the measured $\left(G_{\mathrm{BP}}-G_{\mathrm{RP}}\right)_{0}$ color.

2. Faint regime: For stars fainter than the dashed line (apparent $G=18.5 \mathrm{mag}$ translated to absolute magnitude), or stars that lack a $G_{\mathrm{BP}}$ or $G_{\mathrm{RP}}$ magnitude, we cannot rely on the $\left(G_{\mathrm{BP}}-G_{\mathrm{RP}}\right)_{0}$ color to do a deprojection. Instead, we simply keep the calculated $M_{G_{0}}$ value as it is, and define $M_{G_{0, \text { proj }}}$ to be equal to it. This is therefore a regime where we do not account for unresolved companions.

3. Intermediate regime: For stars in between the dotted and dashed lines $(17.5 \leqslant G \leqslant 18.5$ mag range translated to absolute magnitude), we do an intermediate, ramp-like calculation. We calculate the values we would have obtained following the bright and faint regimes separately, and combine them in a progressive way with linear weighting. In this way, the extremes match the corresponding methods, effectively avoiding a sharp transition between the different regimes.

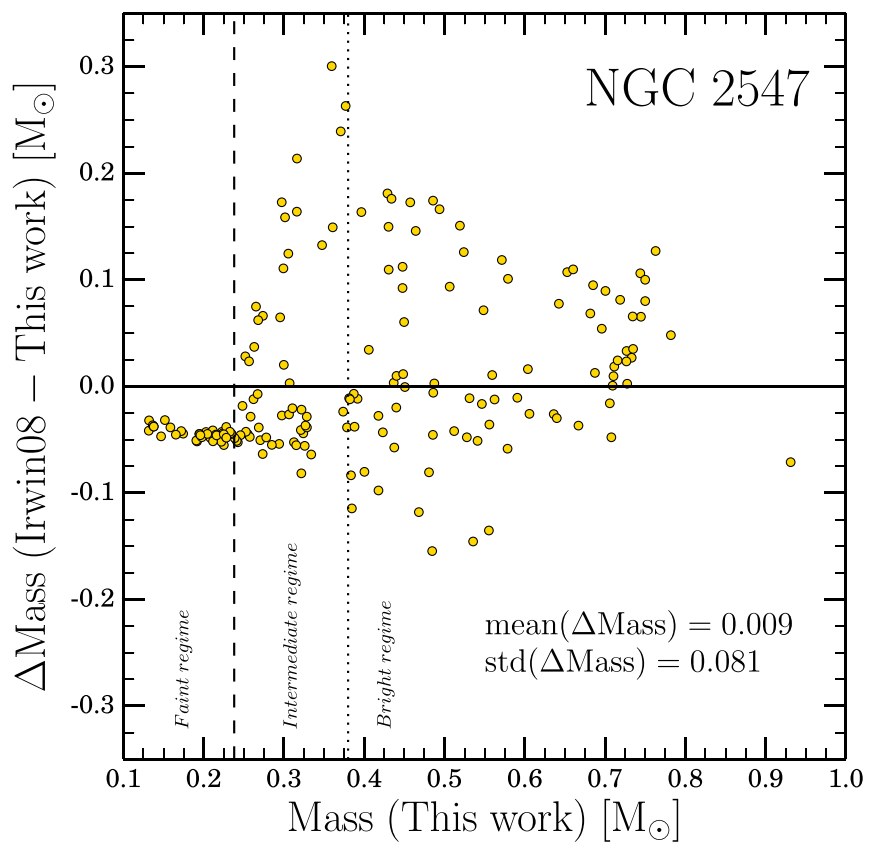

Figure 7. NGC 2547: comparison of our mass estimates with those of Irwin et al. (2008) for the stars in the periodic sample. We show mass difference (in the sense of Irwin et al. 2008 minus this work) vs. mass from this work. Both estimates come from entirely independent photometric data sets and interpolated models. The dotted and dashed lines correspond to the apparent $G=17.5$ and 18.5 mag limits from Figure 3 translated to mass coordinates, and these values define the three regimes used for our mass calculation. We find a good overall agreement, and the comparison suggests a possible systematic uncertainty of $\sim 0.05 M_{\odot}$ in the masses derived by this method. In the intermediate and bright regimes, the Irwin et al. (2008) masses tend to be higher due to their method not accounting for photometric binaries.

For every star, this procedure collapses the observed photometry to the single quantity $M_{G_{0, \text { proj }}}$. We use this value to interpolate the corresponding NGC 2547 PARSEC model in the magnitude-mass and magnitude-temperature space. We only interpolate in the regime of stars fainter than the mainsequence turnoff (i.e., we do not report masses for evolved stars) and down to the faintest $M_{G_{0}}$ magnitude allowed by the model. We report our derived masses and temperatures for the NGC 2547 probable and possible members in Appendix C.

For the subset of NGC 2547 stars with period measurements, Irwin et al. (2008) calculated masses using their I-band magnitudes and the NextGen models (Baraffe et al. 1998). These values offer a completely independent reference point to validate our own masses, and we compare our estimates with theirs in Figure 7. It is important to highlight one key difference between both methods: we account for the presence of photometric binaries (when possible), while Irwin et al. (2008) do not. Accordingly, we find the mass difference to be a strong function of mass itself. For stars in the faint regime, where we do not account for binaries, we see a good agreement but with an approximately constant offset of $\Delta$ Mass $\approx$ $-0.05 M_{\odot}$ that is likely due to the different underlying models used in the interpolation. For higher masses, the scatter increases and many stars have positive mass differences, indicating cases where Irwin et al. (2008) did not account for the contribution of unresolved companions. Overall, we find a good agreement, with mean and standard deviation values of $\Delta$ Mass being $\approx 0.01$ and $0.08 M_{\odot}$, respectively.

The above comparison validates our method and suggests that masses derived in this way are subject to a systematic 
uncertainty of order $\sim 0.05 M_{\odot}$. Statistical uncertainties are of order $\sim 0.01-0.02 M_{\odot}$, considerably smaller than the systematic ones. More importantly, by using the Gaia photometry, we have defined a procedure to calculate the masses and temperatures that can be uniformly applied to all our clusters.

Analogously to Figure 6 for NGC 2547, Figure 8 shows the extinction-corrected absolute CMD for the probable and possible members of all the clusters. Some discrepancies arise for low-mass stars, where the probable members appear brighter/redder than the model (e.g., see the $\sim 0.4 M_{\odot}$ stars in Pleiades and Praesepe). As other studies suggest, these differences could arise due to the known radius inflation problem in cool dwarfs (e.g., Torres \& Ribas 2002; Clausen et al. 2009; Torres et al. 2010; Kraus et al. 2011; Somers \& Stassun 2017; Jackson et al. 2018, 2019). Accounting for the underlying physical process that causes this in the models is beyond the scope of this paper, but we refer interested readers to the works by Somers \& Pinsonneault (2015) and Somers et al. (2020). Regardless, while evidently not perfect, Figure 8 shows a good overall agreement of the data with models across the HR diagram for all the clusters.

We follow the procedure described for NGC 2547, and use the absolute CMDs to calculate masses and temperatures for the probable and possible cluster members. We report these values in Appendix C. For the subset of stars with independent mass and temperature estimates from other studies, we compare these values with ours in Appendix E. Globally, we find our masses and temperatures to be in good agreement with those reported by the period references. The comparisons suggest possible systematic uncertainties of $\sim 0.05-0.1 M_{\odot}$ in mass and $\sim 150 \mathrm{~K}$ in temperature, which are modest considering the different methods, photometric data, and underlying stellar models employed.

\section{Results}

For the remainder of this paper, we focus on the subset of main-sequence stars with period measurements-the periodic samples. In particular, in this section we proceed to investigate how the clusters' rotational sequences change when we consider the astrometric classifications previously derived, to what extent field contamination is an issue, and whether rotational outliers are real cluster members. For this, we remove from our sample all the Pleiades and Praesepe stars that Rebull et al. (2016b, 2017) classify as pulsators, as well as the giant NGC 6811 stars for which Santos et al. (2021) report rotation periods (which are longer than 100 days). Additionally, for the Pleiades and Praesepe stars with multiple period measurements, we only consider their main periods as the adopted rotation period ( $P$ 1 from Rebull et al. 2016a, 2016b and Rebull et al. 2017). Tables with the membership classifications and periods, as well as other important parameters (e.g., Gaia DR2 IDs, derived masses and temperatures, membership probabilities) for the periodic samples are reported in Appendix F.

Figure 9 shows the results of applying our astrometric classification to the rotational sequences of the periodic samples. For each cluster, we show both the literature sequence (i.e., pre-Gaia DR2 membership analysis), and its revised version reported in this work (i.e., post-Gaia DR2). The literature rotational sequences (left column) include all the candidate cluster members reported in the original period references, and we color-code them according to our astrometric classifications. The revised sequences (right
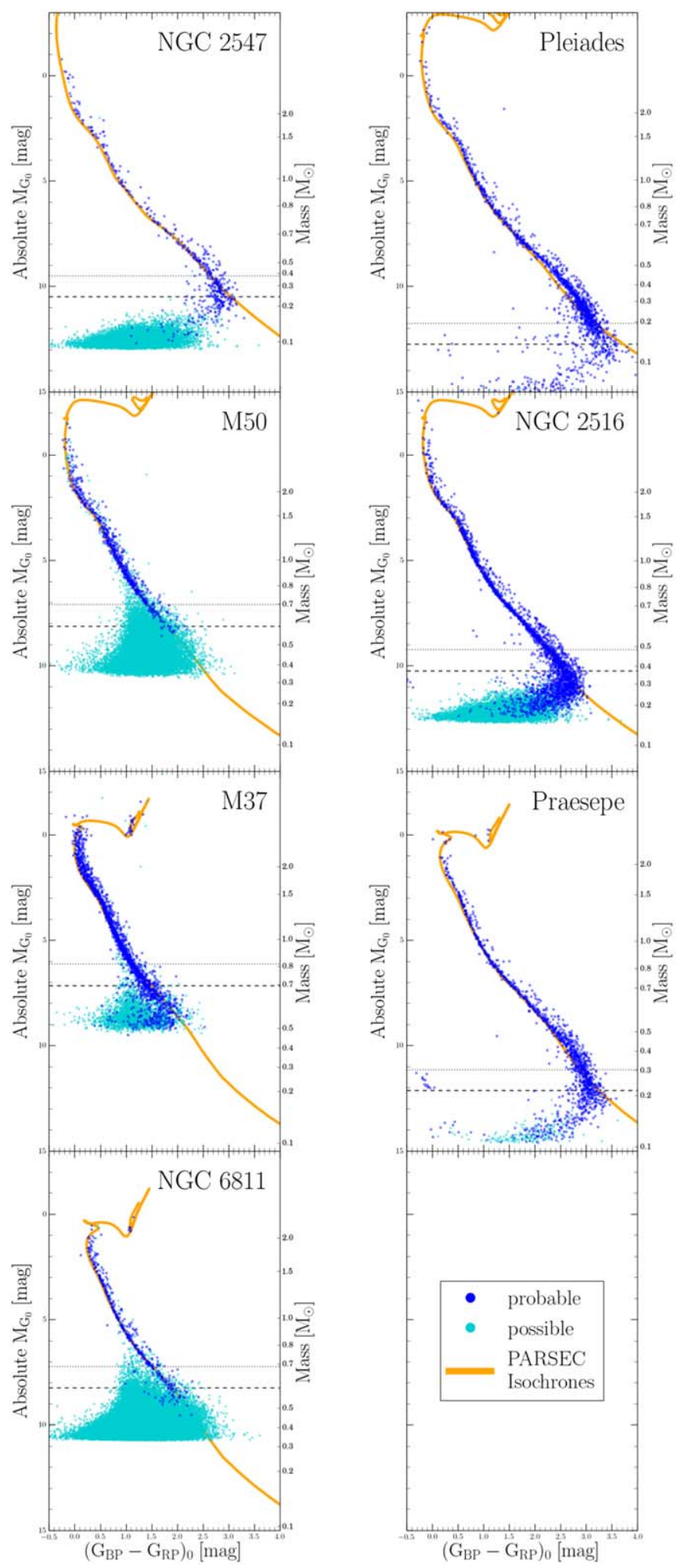

Figure 8. Absolute and dereddened CMD of the probable (blue) and possible (cyan) members for all the clusters we study, analogous to Figure 6. The orange lines are the respective best-fit PARSEC isochrones.

column), only show probable members, possible members, and no info stars, but do not show stars classified as non members. Because at this point we have no means to either confirm or reject the membership status of the no info stars, we simply add them to the possible members category in the revised sequences of Figure 9. 

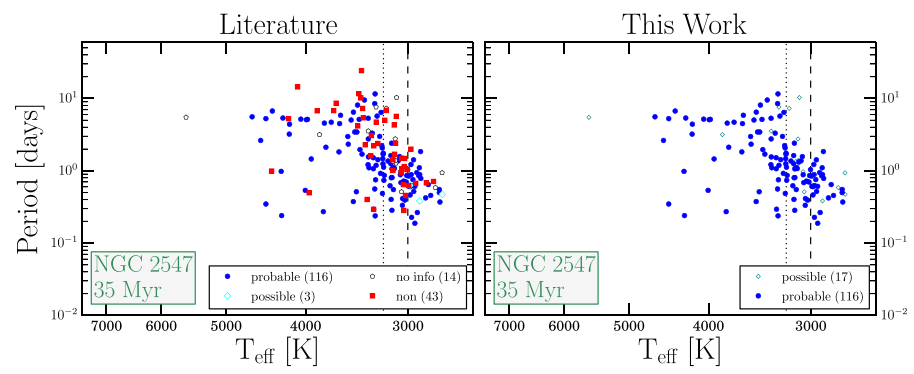

a)
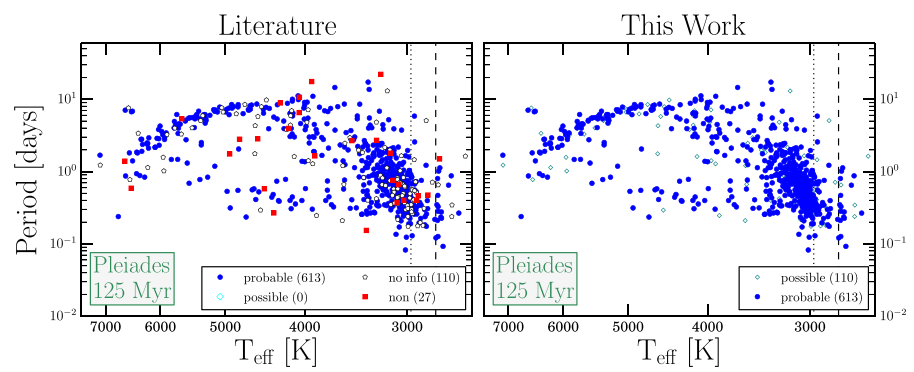

b)
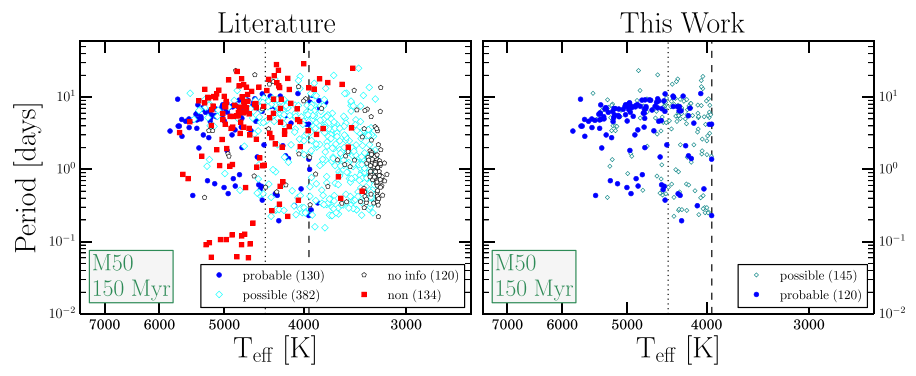

c)
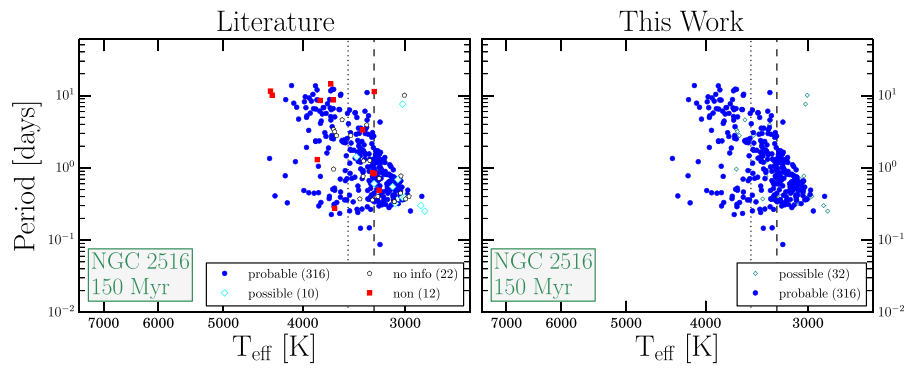

d)
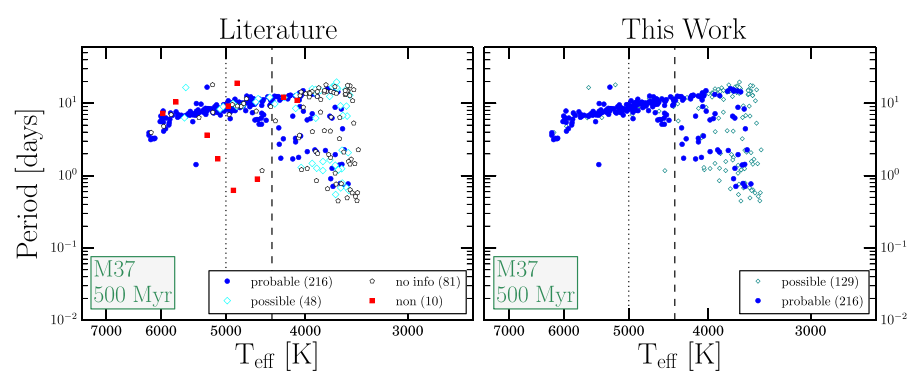

e)
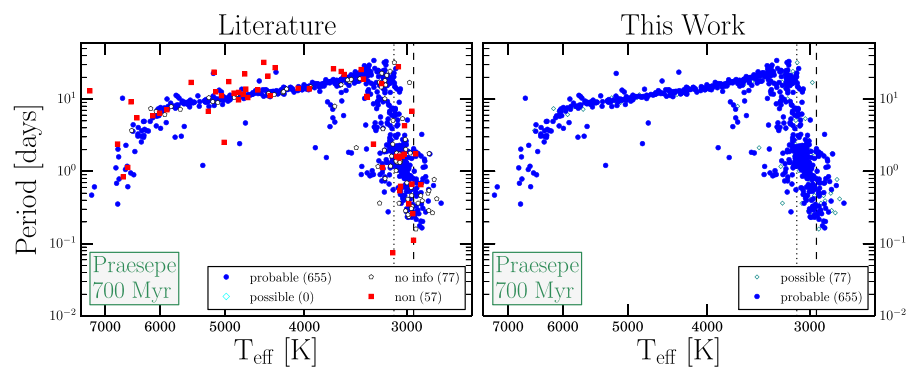

f)
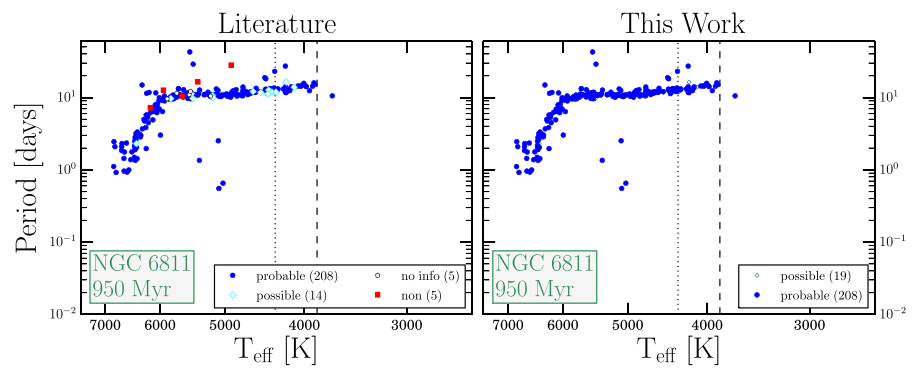

g)

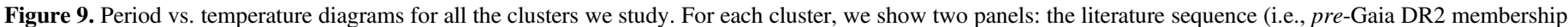

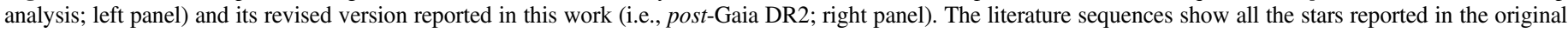

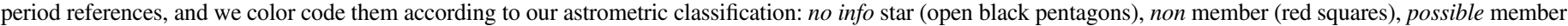

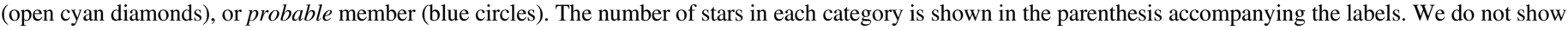

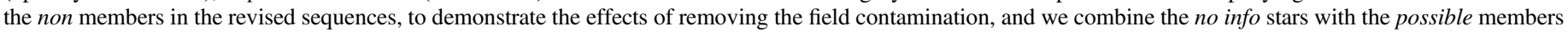

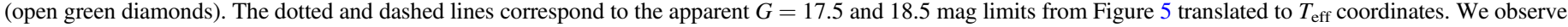

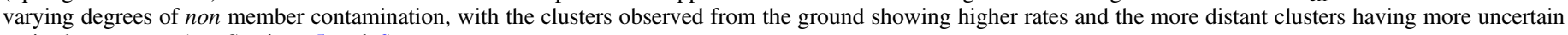
revised sequences (see Sections 5 and 6).

Before comparing the literature and revised rotational sequences in detail, we note that a strong artifact inherited from the Gaia astrometry is present in the membership classification of our most distant clusters. In M50 (distance of $\sim 970 \mathrm{pc}$ ), for $T_{\text {eff }} \gtrsim 4500 \mathrm{~K}$, most of the stars are classified as either probable or non members. On the other hand, stars cooler than this limit are mostly classified as possible members (and furthermore, for $T_{\text {eff }} \lesssim 3500 \mathrm{~K}$ the predominant category is no info stars). This is a direct consequence of the quality of the Gaia astrometry decreasing monotonically for fainter, cooler targets, where our classification method can no longer reliably identify probable or non members, and we instead classify most stars as possible members. A similar effect is observed in M37 (distance of $\sim 1440 \mathrm{pc}$ ), with the limit being around $T_{\text {eff }} \simeq 4000 \mathrm{~K}$. This effect is absent in the rotational sequence of NGC 6811 (distance of $\sim 1080$ pc), but only 
because the periodic sample does not extend to such faint, cool stars.

We now examine Figure 9 in detail and discuss how the inclusion of the astrometric information affects the rotational sequence of each cluster independently:

1. NGC 2547: for this cluster, we classify $\sim 25 \%$ of the Irwin et al. (2008) candidates as non members. By removing this contamination, the rotational sequence changes considerably. At a given $T_{\text {eff }}$, most of the non members actually correspond to stars with the longest periods, and after including our membership analysis, only two stars with period $>10$ days remain (one of them a probable member, while the other is a no info star). Interestingly, the revised NGC 2547 sequence exhibits strong $T_{\text {eff }}$ and mass-dependent trends in the period distribution, with the mean period decreasing with decreasing $T_{\text {eff }}$ for $T_{\text {eff }}<3500 \mathrm{~K}$. Given its young age ( $\sim 35 \mathrm{Myr}$ ), we expect the period versus $T_{\text {eff }}$ distribution of NGC 2547 to be strongly affected by processes that regulate stellar rotation at birth.

2. Pleiades: we find the non member contamination to be small ( $\lesssim 4 \%)$, and that removing it, for the most part, does not empty specific regions in the rotational sequence. This finding is not surprising, as the Pleiades stars studied with K2 by Rebull et al. (2016a, 2016b) and Stauffer et al. (2016) had been previously vetted by proper motion surveys. Further examination of the stars classified as non members reveals that $\approx 70 \%$ of them have $\Delta_{i}$ values between 3 and 5 (i.e., they are outside the cluster's $3 \sigma$ ellipsoid in phase space, but within $5 \sigma$ ), suggesting that a less stringent astrometric selection could have classified them as possible cluster members. Additionally, for the Pleiades, we observe an interesting phenomenon (also observed in Praesepe): there is a large number of no info stars across the entire $T_{\text {eff }}$ range. This is noticeably different from the cases of M50 and M37, where the no info stars are heavily concentrated at the faint, cool end of the distributions. We further discuss this in Section 6, but in short, these no info stars mainly correspond to photometric binaries, which points to biases in the Gaia DR2 astrometry.

3. M50: this cluster shows the largest differences of all when we compare the literature and revised rotational sequences. For $T_{\text {eff }}>4500 \mathrm{~K}$, we find the literature sequence to have substantial non member contamination $(\approx 36 \%)$, and we observe that the different astrometric populations occupy particular regions of the rotational sequence. First, we note that all of the stars with periods $\lesssim 0.1$ day are cleanly identified as non members. This is perhaps not surprising, as these periods most certainly do not correspond to rotational signals but rather pulsation signals from field stars. Second, we note that most of the long-period stars ( $\gtrsim 10$ days) are actually classified as non members. Third, after removing the field contamination, the probable members do form a rather clean sequence of rotation period as a function of $T_{\text {eff }}$, and both branches of slowly and rapidly rotating stars observed in the Pleiades can now be seen in the revised M50 sequence. For $T_{\text {eff }} \lesssim 4000 \mathrm{~K}$, the quality of the Gaia DR2 astrometry does not allow us to classify stars reliably, but we expect the contamination fraction to have significance similar to or higher than that of stars with $T_{\text {eff }}>4500 \mathrm{~K}$. Given how uncertain this part of the diagram is, in the analysis that follows we simply discard the M50 stars cooler than $\sim 4000 \mathrm{~K}$ (vertical dashed line in Figure 9, i.e., the apparent $G=18.5 \mathrm{mag}$ limit translated to $T_{\text {eff }}$ coordinate), and only consider the stars hotter than this limit.

4. NGC 2516: this cluster shows a very low contamination rate, with only $\approx 3 \%$ of the stars being classified as non members, and $\approx 88 \%$ of the Irwin et al. (2007b) stars being classified as probable members. We suspect this arises from the fact that this cluster is very rich and therefore dominates (in terms of number counts; see Figure 5) over the field population in the CMD selection done by Irwin et al. (2007b). Accordingly, both literature and revised rotational sequences are almost identical, with the period distribution showing strong massdependent trends. We note, however, that the periodic NGC 2516 stars span a narrow range in temperature (and mass), and the sample is limited to $T_{\text {eff }}<4400 \mathrm{~K}$ $\left(M<0.7 M_{\odot}\right)$.

5. M37: for $T_{\text {eff }} \gtrsim 4000 \mathrm{~K}$, we find the non member contamination to be $\approx 4 \%$, which is small compared to the $\sim 20 \%$ contamination rate expected by Hartman et al. (2008b). Similarly to NGC 2516, we think this arises from the richness of the cluster compared to the field, in addition to the double CMD selection done by Hartman et al. (2008b). Interestingly, however, the locations of many of the non members do not seem to be arbitrary. Most of them are stars that lie well off of the converged period sequence (at both longer and shorter periods), and are clearly separated from the probable members in the rotational sequence. This result implies that the converged sequence at this age ( 500 Myr; Section 4$)$ is actually stronger than previously thought. We note, nonetheless, that a few rotational outliers still survive the astrometric selection at both longer and shorter periods than the converged sequence. Additionally, and similarly to M50, M37 also exhibits a clear break in the astrometric classification, in this case around $4000 \mathrm{~K}$. For stars cooler than this limit, our method cannot confidently separate probable members from non members, and we instead classify most of them as possible members or no info stars. Nevertheless, and in contrast to M50, given how clean the Hartman et al. (2008b) membership was in the $T_{\text {eff }} \gtrsim 4000 \mathrm{~K}$ regime, we expect most of the stars cooler than this value to be real M37 members, and we do include them in the analysis that follows.

6. Praesepe: the non members correspond to $\approx 7 \%$ of the sample, and this small contamination rate is unsurprising given the previous CMD and proper motion vetting in the K2 stars studied by Rebull et al. (2017). Further examination of these non member stars reveals that $\approx 40 \%$ of them have $\Delta_{i}$ values between 3 and 5 , and therefore a less stringent astrometric selection could have classified them as possible cluster members. Similarly to the Pleiades, the non members do not seem to empty specific regions in the rotational sequence, with the exception of the stars located in the $4300<T_{\text {eff }}<5500 \mathrm{~K}$ range. Many of these non members have longer periods than the converged sequence, and the revised rotational sequence appears narrower than previously thought for this $T_{\text {eff }}$ range at this age ( $\sim 700 \mathrm{Myr}$; Section 4$)$. We find this result to be similar to that of M37, hinting at a 
convergence of rotation rates that is considerably stronger than previously thought (see Section 6). Nonetheless, we also note that a few rotational outliers survive the astrometric analysis and are still present in the revised sequence. Finally, and similarly to the Pleiades, we find that the no info Praesepe stars populate the entire $T_{\text {eff }}$ range of the sequence. These stars mostly appear as photometric binaries in the CMD, and we further discuss them in Section 6.

7. NGC 6811: this cluster shows a very low contamination rate, with only $\approx 2 \%$ of the stars being classified as non members. This is consistent with expectations, given the samples that the NGC 6811 rotation period catalogs were based on. Meibom et al. (2011a) vetted stars on the basis of RV data, Curtis et al. (2019a) combined CMD selections with a set of astrometric cuts to ensure consistency with the cluster's phase-space projections (although they did not take the stars' uncertainties or intrinsic cluster dispersion into account), and Santos et al. (2021) performed their lightcurve analysis based on our sample of probable members. While all of the above work together to produce a low contamination rate by construction, three of the five stars classified as non members are clear outliers when compared with the probable members sequence. Similar to what we see in the other old clusters (M37 and Praesepe), however, the revised sequence does show a number of long-period outliers, hinting at a rare channel that produces slow rotation for a small fraction of members in $\gtrsim 0.5$ Gyr old clusters.

Finally, we point out a feature that is ubiquitous in all the clusters we study: the revised rotational sequences show a number of rapid rotators that survive all the astrometric cuts. These are stars that likely correspond to synchronized binaries, and they stand out more clearly in the older M37, Praesepe, and NGC 6811 clusters with periods of 0.5-2 days and $5000<T_{\text {eff }}<6000 \mathrm{~K}$.

After having analyzed how the clusters' rotational sequences change when the non member contamination is excluded, we emphasize that properly accounting for cluster membership remains a fundamental piece of empirical studies of stellar rotation. Incorrect or biased conclusions could be derived from rotational sequences that lack the appropriate vetting. Replicating our analysis with improved astrometry should further refine the astrometric classifications, and expand the mass and temperature range where the probable and non members can be reliably distinguished from each other.

\section{Discussion}

In this section, we use the periodic samples to study stellar rotation as a function of mass and age.

\subsection{Rotation Data in the Gaia Era}

At this point, for every cluster in our sample, we have classified the periodic stars using our astrometric analysis (Section 3), presented a set of revised properties that are needed to perform meaningful inter-cluster comparisons (Section 4), and discussed the effects that removing the field contamination has on the individual rotational sequences (Section 5). Now, we combine all of these to construct an updated portrait of the evolution of stellar rotation.

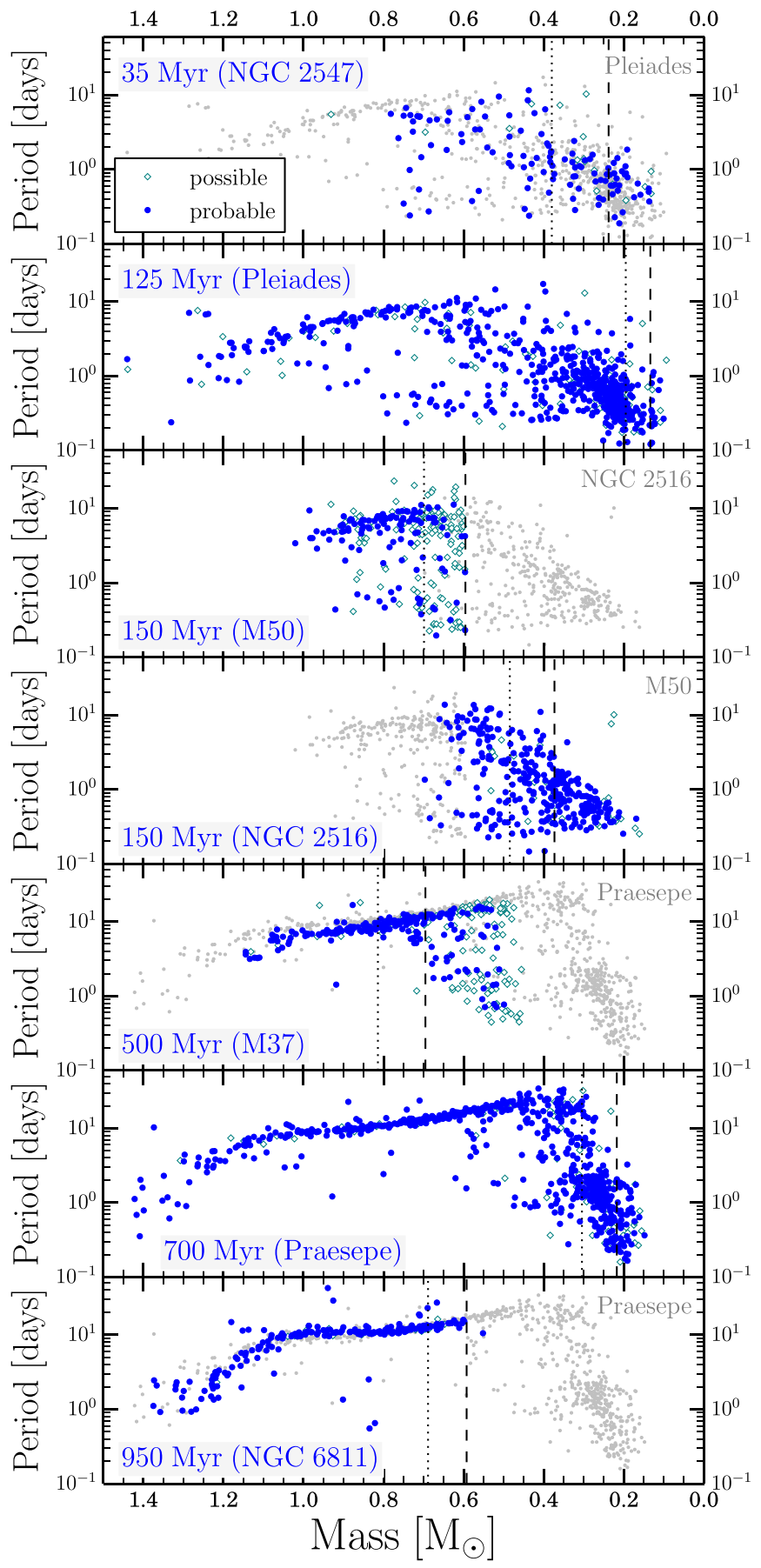

Figure 10. Revised period vs. mass diagrams for all the clusters we study (i.e., after having removed the non member contamination). The clusters are sorted by age, and the ages and masses are the values calculated in Section 4.2. The points are color-coded in the same fashion as the revised sequences of Figure 9. The dotted and dashed lines correspond to the apparent $G=17.5$ and $18.5 \mathrm{mag}$ limits from Figure 5, translated to mass coordinates. To aid inter-cluster comparisons, in some panels we add an additional cluster in gray in the background. This figure represents state-of-the-art data for studies of stellar rotation (see Section 6.1)

We illustrate this in Figure 10, where we show the revised period versus mass diagrams as a function of age. The colorcoding is the same as the revised sequences of Figure 9, and the non member contaminants have been removed. This represents the state of the art for rotation studies in terms of clean samples with stellar masses, temperatures, and ages derived in a consistent scale. We make these data publicly available in Appendix F. 


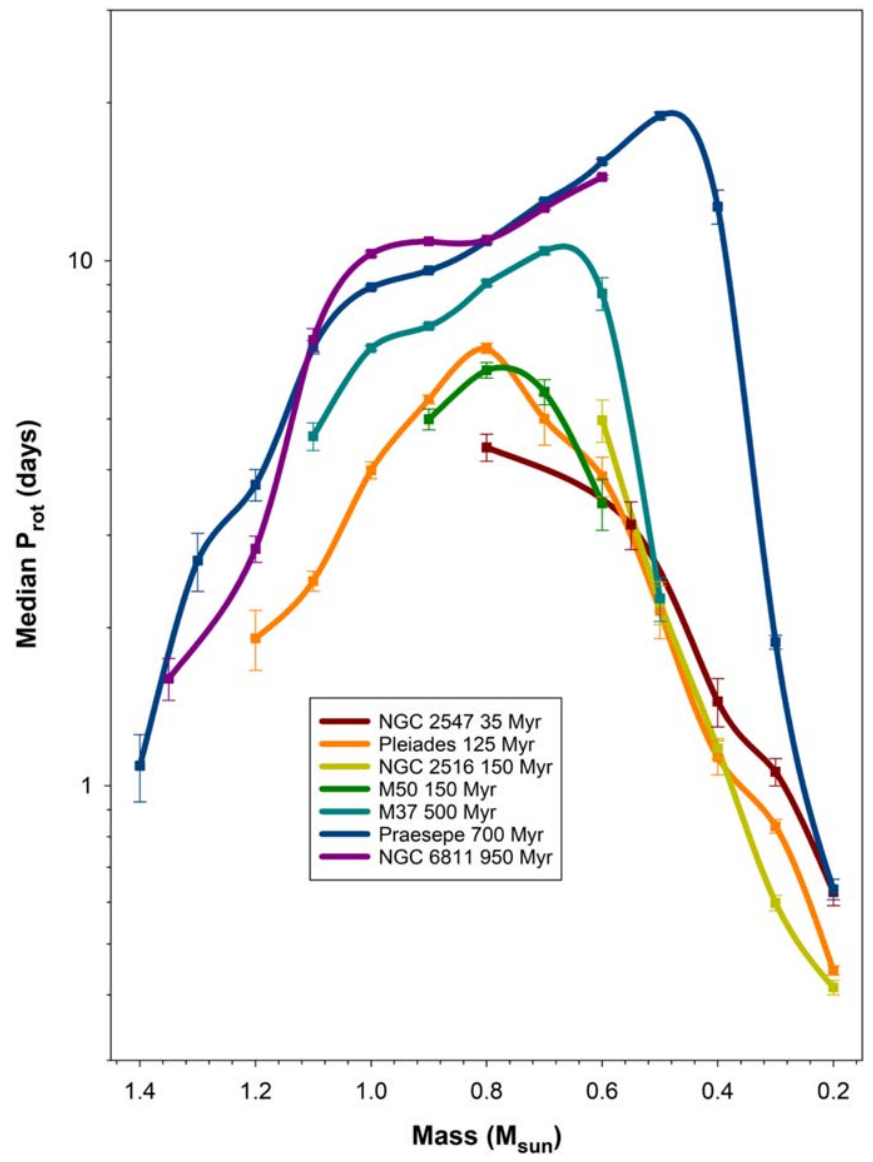

Figure 11. Period vs. mass diagram for the median rotators. The squares show the median periods and their standard errors, computed in $0.1 M_{\odot}$ bins. The solid lines show a spline fit to the data. Each cluster is shown as its own line, with the names, revised ages, and colors indicated in the legend. For masses $<0.5 M_{\odot}$, the median rotators have a non-monotonic behavior, showing a spinup from ages $\sim 35$ to $125-150 \mathrm{Myr}$, and a spin-down afterward at $\sim 700 \mathrm{Myr}$. For masses $>0.5 M_{\odot}$, they show a monotonic spin-down as a function of time until $\sim 700$ Myr, after which the behavior becomes complicated (see Section 6.2).

One of the major results of our study can be seen in the rotational sequence of M50 in Figure 10. When the non member contamination is removed, the revised M50 sequence clearly resembles that of the Pleiades, as we would expect given their similar ages (150 and $125 \mathrm{Myr}$, respectively). Incidentally, the low-mass end of the M50 sequence coincides with the highmass end of the co-eval NGC 2516 sequence $\left(\approx 0.65 M_{\odot}\right)$. This is illustrated in their respective panels, where we show the M50 sequence in blue and green while the NGC 2516 sequence is shown in gray, and vice versa. Given their indistinguishable ages, the merged M50 and NGC 2516 data can provide a valuable comparison point for the benchmark Pleiades cluster. Likewise, a similar comparison can be made for the older M37 and Praesepe clusters. Ultimately, the above demonstrates a decisive finding: when careful membership analysis are performed, the rotational sequences that have been constructed from ground-based observations (e.g., M50 by Irwin et al. 2009) can be as informative for stellar rotation studies as those constructed from space-based observations (e.g., the Pleiades by Rebull et al. 2016a). In this context, future dedicated groundbased monitoring combined with careful astrometric selections using Gaia could provide unprecedented constraints for angular momentum evolution (e.g., Curtis et al. 2020). This conclusion is particularly relevant in the post Kepler and $\mathrm{K} 2$ era, and considering that only a fraction of the TESS targets are being observed with long baselines.

Regarding the field contaminants, our membership analysis yields varying degrees of contamination rates. As mentioned in Section 2, we expected the clusters observed from the ground to show higher contamination rates compared to those observed from space. This turns out to be the case for NGC 2547 and M50, where we find the contamination to be $\approx 25 \%$ and $36 \%$, respectively. While high, these values are lower than the $\sim 40 \%-60 \%$ rates anticipated by Irwin et al. (2008, 2009), which were based on predictions from Galactic models. For the rest of the sample, we find the contamination rates to be lower, on the order of $\lesssim 5 \%$ (see Section 5).

Interestingly, although our membership study has predominantly removed rotational outliers at both long and short periods for several clusters, many outliers are nonetheless classified as probable members and remain in the revised sequences. An interesting example of this is provided by the slowly rotating stars (periods $\approx 7-10$ days) in the young NGC 2547 (age of $\sim 35 \mathrm{Myr}$ ), hinting at strongly mass-dependent initial conditions for stellar rotation (see also Somers et al. 2017; Rebull et al. 2018, 2020). Similarly, all three $\gtrsim 0.5 \mathrm{Gyr}$ old clusters show confirmed members that are rotating faster $(\sim 1$ day) and slower ( $\sim 20$ days) than their slowly rotating branches. Now that the membership statuses of these outliers have been confirmed, their presence can no longer be ignored or attributed to field contaminants.

The rapid rotators in systems with a converged sequence cannot be explained by single star evolution in current models. The most plausible explanation is that they have experienced tidal synchronization or are merger products. Regarding the slowly rotating outliers, a quick inspection reveals that most appear as typical cluster members in astrometric and photometric regards, but a fraction of them are photometric binaries in the CMDs. ${ }^{10}$ We suspect that they could correspond to the long-period end of the distribution of tidally synchronized binaries (e.g., see Lurie et al. 2017). Another explanation could be that they were born with unusually low angular momentum, but given that the young Pleiades sequence does not show many of these stars, we find this hypothesis less likely. Ultimately, unless explained by modern theories of angular momentum evolution, these outliers could potentially weaken the applicability of gyrochronology in field stars.

\subsection{A Revised Picture of Angular Momentum Evolution}

We now quantify the trends that can be obtained from the revised sequences of Figure 10. We display this in three different approaches, and these are shown in Figures 11-13. Additionally, we calculate the percentiles of the rotational distributions of our clusters in different mass bins, and we make them publicly available in Appendix G.

Figure 11 shows, for each cluster in our sample, the period versus mass diagram of the median rotators in $0.1 M_{\odot}$ bins. This allows us to simultaneously analyze the mass and age dependence of stellar rotation. For stars with masses below $0.5 M_{\odot}$, the median rotation periods for the young NGC 2547 (35 Myr) are longer than those seen in the older Pleiades and NGC 2516 clusters (125 and $150 \mathrm{Myr}$, respectively). This is

\footnotetext{
${ }^{10}$ Particularly EPIC 211898294 in Praesepe, and KIC 9349106 and KIC 9656987 in NGC 6811.
} 

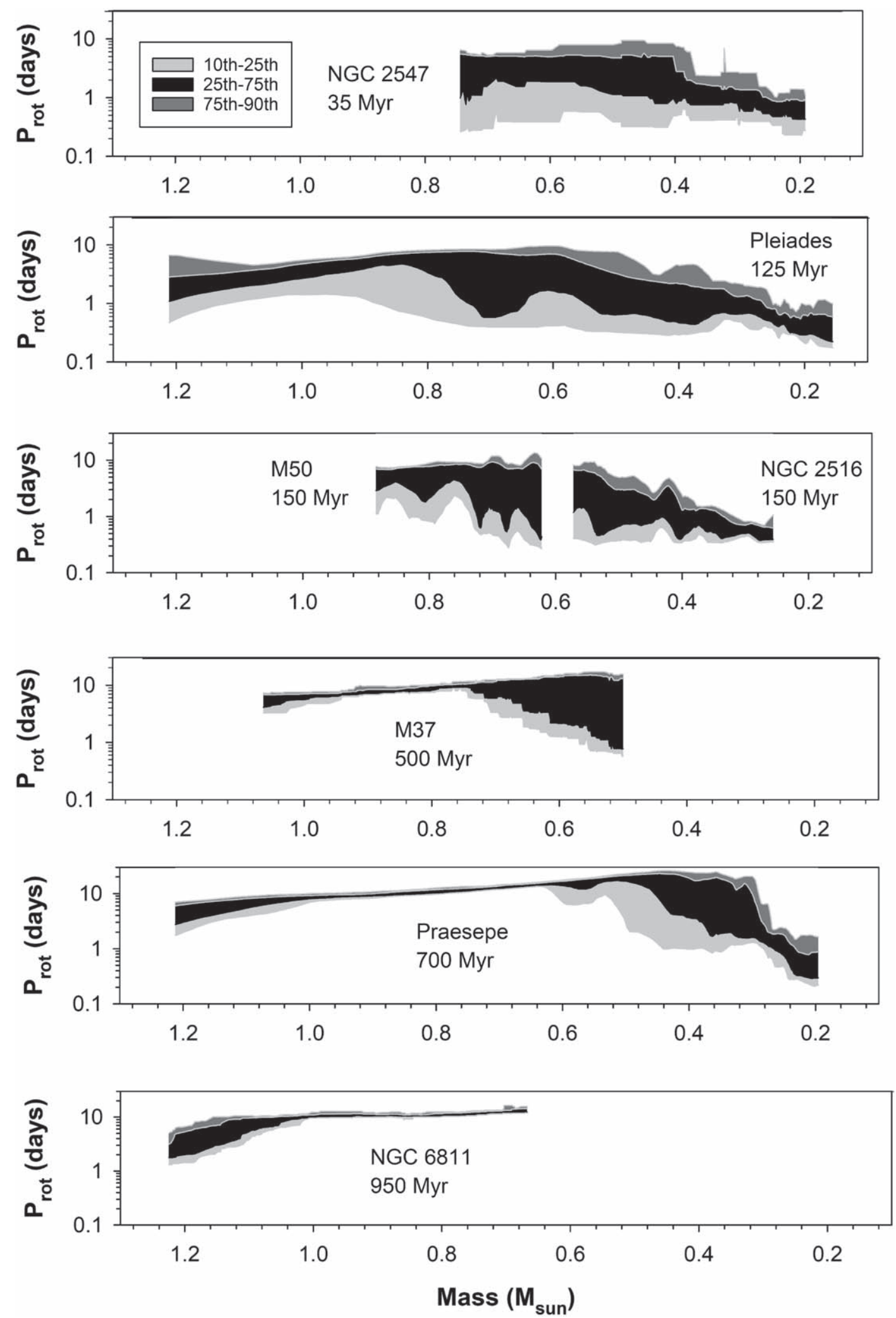

Figure 12. Distribution of rotation rates as a function of mass for the clusters considered in our sample. The 10th, 25th, 75th, and 90th percentiles of the period distributions are computed for 40 star bins in data rank ordered by mass, and then boxcar-smoothed. The region of rapid rotators is shown in light gray, that of intermediate rotators is shown in black, and that of slow rotators is shown in dark gray. M50 and NGC 2516 are shown in a combined panel, given their virtually identical ages and complementary mass ranges. We see a clear convergence of rotation rates into a narrow sequence in the older clusters, with the limit (i.e., lowest mass) of this converged branch progressively extending to lower masses for older ages (e.g., $\sim 0.85 M_{\odot}$ in the Pleiades to $\sim 0.65 M_{\odot}$ in Praesepe). For masses below this, the interquartile range is actually broader in the older clusters than in the younger ones. This suggests that, at fixed mass, the rapid rotators lose less angular momentum (or experience a lower torque) than the slow rotators. Current models of angular momentum evolution do not reproduce this feature. 

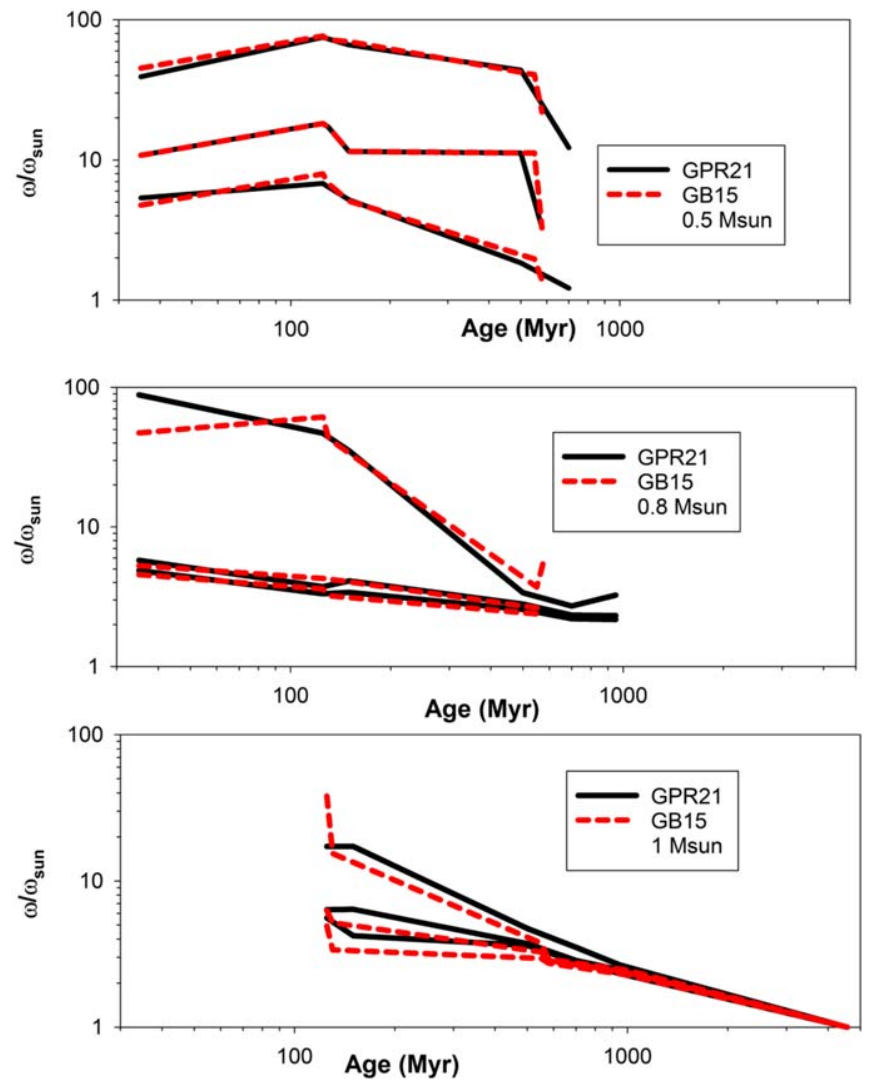

Figure 13. Temporal evolution of the 25th, 50th, and 90th percentiles of the angular velocity distributions (normalized to the solar value). We show this for different masses, with $0.5 M_{\odot}$ stars shown in the top, $0.8 M_{\odot}$ stars shown in the middle, and $1 M_{\odot}$ stars shown in the bottom. Our values are shown as the black solid line (GPR21), and the literature values from Gallet \& Bouvier (2015) are shown as the red dashed line (GB15). Our analyses of the clusters' memberships and properties play a role in shifting our values with respect to the literature ones in both axes (see Section 6.2).

consistent with expectations, considering that stars of these masses take a few hundred million years to reach the ZAMS, and are therefore still contracting by the age of NGC 2547. By the age of Praesepe (700 Myr), these stars have spun down in a strongly mass-dependent fashion, with lowest masses still showing periods below 1 day, and $\sim 0.4 M_{\odot}$ stars rotating at $\gtrsim 10$ days.

The stars more massive than $0.5 M_{\odot}$, on the other hand, show a monotonic behavior with time in most of the age range probed by our clusters. They consistently spin down as they age from 35 to $700 \mathrm{Myr}$, and their median rotator trends in Figure 11 do not intersect each other, in agreement with expectations from a Skumanich-type spin-down (i.e., period $\propto \operatorname{age}^{1 / 2}$ ). The notable exception to this, however, is the comparison of Praesepe and NGC 6811. For these two clusters, their rotational sequences do not seem to be simple translations of each other to longer or shorter periods. Although their age difference is non-negligible ( $\sim 250 \mathrm{Myr}$ ), their median rotators are virtually overlapping at $\sim 1.1 M_{\odot}$ and in the $0.8-0.6 M_{\odot}$ range. The latter of these has already been noted by Curtis et al. (2019a) (see also Meibom et al. 2011a), and a similar overlapping was reported by Agüeros et al. (2018) for a smaller sample in the 1.4 Gyr old cluster NGC 752. In terms of the interpretation of this feature, Aguieros et al. (2018) and Curtis et al. (2019a) have formulated it as a temporary epoch of stalling in the spin-down of $\mathrm{K}$ dwarfs, and
Spada \& Lanzafame (2020) have proposed that it arises from the competing effect between magnetic braking and a strongly massdependent internal redistribution of angular momentum. We leave a detailed examination of this as future work, but given the considerable difference in metallicity between both clusters $([\mathrm{Fe} / \mathrm{H}]=+0.16 \mathrm{dex}$ for Praesepe and $+0.03 \mathrm{dex}$ for NGC 6811; Netopil et al. 2016), we highlight the importance of incorporating chemical composition in comprehensive models of angular momentum evolution (e.g., Amard \& Matt 2020; Amard et al. 2020; Claytor et al. 2020).

Figure 11 can also be used to remark upon global properties of stellar rotation. First, regardless of the age, no sharp transition is seen in the rotation periods near the boundary between partially convective and fully convective stars $\left(\sim 0.35 M_{\odot}\right)$. Second, as illustrated by NGC 2547 in our sample, and by the young Upper Scorpius and Taurus associations in Somers et al. (2017) and Rebull et al. (2018, 2020), the initial conditions of stellar rotation are strongly mass-dependent and need to be accounted for in models of angular momentum evolution. Third, when considering the young ages probed by our sample ( 35-200 Myr), we note that the stars in the $1.0-0.6 M_{\odot}$ range populate a global maximum in terms of rotation periods. In other words, the median rotators in this mass interval never rotate more rapidly than $\sim 3-4$ days. Given the intimate connection between stellar rotation and activity (e.g., Wright et al. 2011, 2018; Lehtinen et al. 2020), where more rapidly rotating stars tend to expose their planets to higher levels of potentially harmful radiation, this feature could provide an optimal window in the search for habitable worlds.

In Figure 12, we show the rotational sequences for the clusters we study, but in this case separating the data into different percentiles of rotation. For each cluster, across the mass range where they have period information, we show a band of rapid rotators (star within the 10th to 25 th percentiles of the period distribution; light gray region), intermediate rotators (25th to 75 th percentiles; black region), and slow rotators (75th to 90th percentiles; dark gray region). At young ages, we observe a well-defined upper limit in the rotation periods, with virtually no stars showing periods longer than $\sim 10$ days. At late ages, we clearly see the convergence of rotation periods to a tight sequence in a heavily massdependent fashion, in agreement with expectations. The convergence in our revised sequences is so strong that the upper and lower edges of the distribution in M37, Praesepe, and NGC 6811 in the $1.0-0.8 M_{\odot}$ range are barely visible. Additionally, in both Figures 11 and 12, there is another important clue about the torques from magnetized winds. For all clusters, there is: (1) a characteristic mass where the distribution has collapsed down into a narrow range (the unsaturated domain, where the torque is $\left.d J / d t \propto \omega^{3}\right)$; and (2) a mass range just below it where stars still retain a range of surface rotation rates (with the rapid rotators still being in the saturated domain, where the torque is $d J / d t \propto \omega)$. For this latter mass range, the width of the interquartile range is broader in the older systems ( $\gtrsim 500 \mathrm{Myr})$ than it is in the young ones $(\lesssim 150$ Myr). This indicates a relative divergence in the surface rotation rates, in contrast to the convergence that is predicted by canonical models of angular momentum loss (e.g., van Saders \& Pinsonneault 2013; Matt et al. 2015).

In other words, the data in Figure 12 appear to require lower torques for rapid rotators than for intermediate rotators, which 
acts to create the bimodal distribution that has been noted in the literature (and which is now clearly seen in our sample). One possible explanation for this, as proposed by Garraffo et al. (2018), is that there is a change in field topology, such that rapid rotators have more complex magnetic field configurations than intermediate rotators and are therefore less efficient at losing angular momentum. Although this concept is interesting, the data could also be fit with a wind model that incorporates a supersaturation mechanism, where the overall field strength declines. Alternatively, it could indicate a dependence of the convective overturn timescale on the rotation rate at fixed mass, as loss laws are typically parameterized by the ratio of the rotation period to this timescale (i.e., the Rossby number). Ultimately, a number of additional physical properties that are involved in the loss of angular momentum could be influencing the empirical sequences (e.g., mass-loss rate, dipole field strength, Alfvén radius). It will be informative to compare different proposed mechanisms, but an adjustment to canonical models is clearly required. We note that the late time behavior of the distribution is not sensitive to the treatment of this feature, as older stars have lost memory of their initial conditions, and typical semi-empirical approaches are tuned to reproduce the spin-down of the slow and rapid rotator branches of the data. However, the properties of intermediate rotators in systems that retain rapid rotators will not be correctly modeled by classical methods.

Finally, in Figure 13, we show the temporal evolution of the rotational percentiles for three different masses $(0.5,0.8$, and $\left.1 M_{\odot}\right)$. We frame this as a comparison with Gallet \& Bouvier $(2015)^{11}$ in terms of the 25th, 50th, and 90th percentiles of the distribution of angular velocities $\left(\omega=2 \pi / P_{\text {rot }}\right)$, normalized to the solar value $\left(\omega_{\odot}=2.87 \times 10^{-6} \mathrm{~s}^{-1}\right)$. Our membership analysis plays a role in shifting our values with respect to the literature ones in both axes, as the revised cluster properties change the ages (Section 4) and the revised rotational sequences change the angular velocity distributions (Section 5). In general, our percentile evolution appear less noisy and shows smoother patterns. In the 0.5 and $0.8 M_{\odot}$ stars, our data capture the same broad trends. Importantly, the overall slow rotation in young stars remains, and this provides further evidence for the need for core-envelope decoupling in angular momentum models (e.g., Denissenkov et al. 2010). Before our work, the classification of all of the slow rotators in NGC 2547 as field contaminants was a real possibility, and while our analysis did remove a number of them, some still remain. For the $1 M_{\odot}$ stars, our data suggests a faster rotation in all percentiles (as our analysis has predominantly removed slow rotators), and a globally steeper spin-down slope that remains approximately constant (and similar to the Skumanich value) in the entire age range.

\subsection{Gaia Biases against Photometric Binaries}

Investigating the behavior of binary stars in empirical rotation studies is important to better understand stellar populations and angular momentum evolution. In the regime of wide separations, binaries can be used to place novel gyrochronology constraints in unexplored age and metallicity domains (Chanamé \& Ramírez 2012; Janes 2017; GodoyRivera \& Chanamé 2018). In the regime of close separations, recent studies have found a correlation between rapid rotation

\footnotetext{
11 Note that no rotational data had been published for the $0.8 M_{\odot}$ stars in NGC 6811 by the time Gallet \& Bouvier (2015) performed their analysis.
}

and field binaries (Simonian et al. 2019, 2020). Specifically in the M-dwarf domain, Stauffer et al. (2018) found that, in the young Upper Scorpius association ( $~ 8 \mathrm{Myr})$ and the Pleiades cluster, the stars with multiple period measurements from K2 are predominantly photometric binaries (see also Rebull et al. 2016a, 2017, and Tokovinin \& Briceño 2018). Furthermore, Stauffer et al. (2018) showed that the rotation periods of these young, unresolved binaries, are much shorter as well as more similar to each other than otherwise expected for single $\mathrm{M}$ dwarfs. This hints at the crucial role that binarity plays in star formation and the rotation rates imprinted at birth (see also Messina 2019).

In this context, in Figure 14, we examine the rotational sequences and CMDs of the no info stars in our most nearby clusters (i.e., the targets that did not pass our astrometric quality cuts; see Section 3.1.3). In the revised rotational sequences of M50 and M37, the stars classified as no info predominantly occupy their very lowest $T_{\text {eff }}$ (and therefore apparent magnitude) values, which indicates that their classification is purely due to low-quality astrometry. In contrast to this, for the Pleiades and Praesepe, the no info stars span the entire $T_{\text {eff }}$ range (see Section 5), and we would have expected them to have high-quality Gaia astrometry. Considering the previous selections made by Rebull et al. (2016a) in the Pleiades and by Rebull et al. (2017) in Praesepe, and given the low contamination rates found for these systems in Section 5, we expect most of these no info stars to be real members of these nearby clusters.

The CMDs of Figure 14 show that the no info stars appear predominantly as photometric binaries with respect to the sequences of probable members in both the Pleiades and Praesepe. This result hints at biases in the Gaia DR2 data against these unresolved systems, even with conservative quality cuts, where their separation is seemingly too close for the current astrometry to properly distinguish their orbital and systemic motions. Interestingly, many of these no info stars appear as rapid rotators in the Pleiades rotational sequence. By the age of Praesepe, however, they are overlapping with the bulk of the probable members on the slowly rotating branch. This indicates that, while their separations are close enough to be unresolved in Gaia, these binaries are not synchronized and they are actually spinning down in a fashion similar to that of the single stars. This finding is similar to that of Stauffer et al. (2018), who reported that the rapid rotation of stars in binaries with respect to single stars is gone by the age of Praesepe, but in this case we extend it to higher-mass stars, beyond the M-dwarf regime.

Figure 14 also provides an interesting distinction in our membership classification. While presumably some of the stars classified in the no info category could turn out to be field contaminants, the stars classified as non members appear to occupy different locations in the rotational sequences and CMDs. Most of these non members appear below the track of probable members on the $\mathrm{CMD}$, and correspond to rotational outliers in the converged branch of Praesepe. In addition to their astrometric distinction, we take this as further confirmation that the non members and no info stars correspond to markedly different populations.

Finally, although our astrometric selections were designed to avoid biases against binary stars, we conclude that newer Gaia data will be needed to fully resolve these photometric binaries. In the meanwhile, although they are not classified as probable 

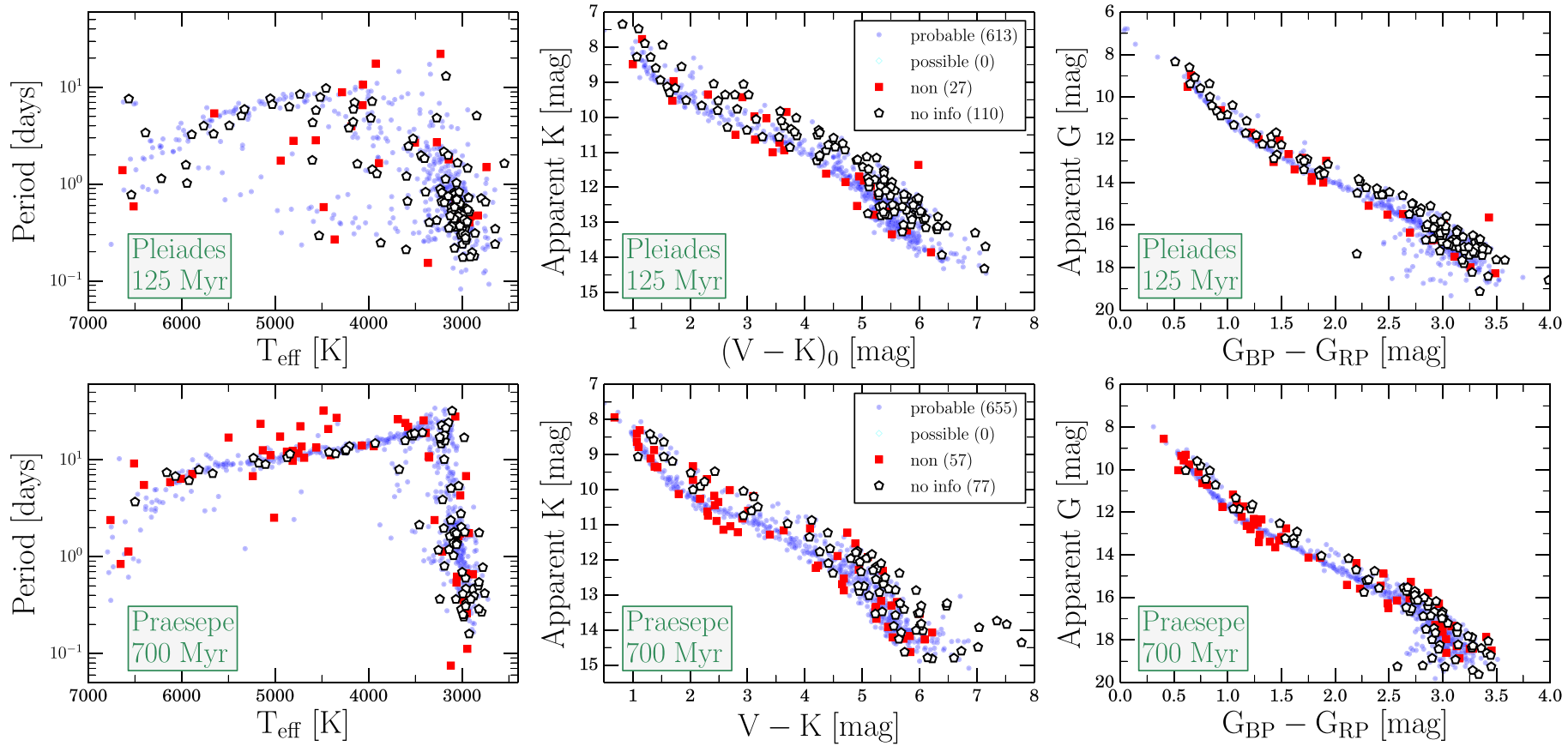

Figure 14. Period vs. temperature (left column), apparent $V$ vs. $V-K \mathrm{CMD}$ (middle column), and apparent $G$ vs. $G_{\mathrm{BP}}-G_{\mathrm{RP}} \mathrm{CMD}$ (right column) for the nearby Pleiades (top row) and Praesepe (bottom row) clusters. We take the $V$ and $K$ photometry from Rebull et al. (2016a, 2017). The color and symbol coding are the same as in the literature sequences of Figure 9. For the purposes of this figure, we highlight the no info stars and non members over the probable members. For both clusters, the stars classified as no info stars appear predominantly as photometric binaries on both CMDs, hinting to biases in the Gaia astrometry for these systems. Notably, while in the $\sim 125$ Myr Pleiades sequence many no info stars correspond to rapid rotators, almost all of them have converged onto the slowly rotating branch in the $\sim 700$ Myr Praesepe sequence. This indicates that, although unresolved, these likely binary systems are spinning down as single stars do (see Section 6.3).

members by our membership analysis, we advise future rotation studies of binary stars to include these no info stars in their samples, for completeness purposes.

\section{Conclusions}

Open clusters have played a key role in stellar rotation studies, but their memberships are often uncertain and the expected contamination rates can reach up to $\sim 60 \%$. In this paper, we use the Gaia DR2 astrometry to revise the memberships of several open clusters with rotational data (Table 1), and provide an updated portrait of stellar rotation as a function of mass, temperature, and age.

For a given cluster, we carry out an unrestricted search for cluster members in the Gaia data by performing astrometric modeling in parallax and proper motion space. From this, we calculate membership probabilities and identify probable cluster members, non members (i.e., field stars), and an intermediate category of possible members (typically faint stars with large astrometric uncertainties). An additional category denominated no info stars is assigned to stars that do not have enough astrometric information to be classified. Although our membership selection only includes astrometric (but not photometric) data, the CMD projections of the probable members show clean isochrone-like sequences for all the clusters (Figure 5).

We use the homogeneous Gaia photometry to derive revised cluster properties (age, distance modulus, and reddening; see Table 2) by comparing with state-of-the-art evolutionary models. Importantly, we find our revised ages to be in good agreement with pre-Gaia literature estimates, and in some cases, in disagreement with more recent analyses (see Section 4). We further exploit the Gaia photometry and compare it with models to calculate stellar masses and temperatures for our probable and possible cluster members. All of this allows us to perform an analysis of the clusters' rotational sequences in a common mass, temperature, and age scale, something that has been missing in previous studies.

We crossmatch the Gaia stars with the rotation period catalogs, and use our membership analysis to remove the non member contaminants from the rotational sequences of the clusters (Figure 9). We find varying degrees of non member contamination, with some of the clusters observed from the ground showing high rates (e.g., $\approx 25 \%$ and $36 \%$ for NGC 2547 and M50), and the clusters observed from space showing lower rates (e.g., $\approx 4 \%$ for the Pleiades). M50 is an exceptional case, where our revised sequence is considerably different from the previous literature one, and its revised sequence now clearly resembles that of the benchmark and similar-age Pleiades cluster.

We present an updated and self-consistent portrait of the evolution of stellar rotation, and quantify important trends that can be obtained from it. Our most important findings in these regards are:

1. Once the non member contamination has been removed, the rotational sequences of clusters observed from the ground can be as constraining as those observed from space (Figure 10). This is particularly relevant for the post Kepler and $\mathrm{K} 2$ era.

2. Although our membership analysis has predominantly removed rotational outliers in all the clusters, many of these still survive at both short and long periods (Figure 10). It is likely that some of these correspond to synchronized binaries (especially the rapid rotators), but most slow rotators appear as typical single stars in regard to astrometry and photometry. An alternate explanation for slow rotators would be that there is a small population 
with very low birth angular momentum. In any case, these stars can no longer be attributed to field contamination, and they need to be explained by future theories of angular momentum evolution.

3. At young ages, stars in the $1.0-0.6 M_{\odot}$ range populate a global maximum in terms of rotation periods. Given the strong rotation-activity connection in stars, this potentially provides their planets with an optimal window for habitability (Figure 11).

4. At the age of the young NGC 2547 cluster ( 35 Myr), there is a clear-cut set of maximum periods ( $\sim 10$ days), which provides clues about the initial conditions of rotation. At old ages ( $\gtrsim 500 \mathrm{Myr})$, stars in the $1.1-0.6 M_{\odot}$ range converge to a sequence that is even narrower than previously observed (Figure 12).

5. In the saturated domain, where stars have not yet converged into a uniform sequence at fixed mass, the separation (in terms of rotation periods) between the intermediate and rapid rotators increases with age. This feature is not predicted by classical models, and we interpret it as the latter group experiencing lower torques compared to the former one (Figure 12).

6. We find biases in the Gaia data against photometric binaries, which predominantly appear as rapid rotators at young ages but nonetheless spin down as single stars do (Figure 14).

The data products of this paper will be a useful reference for the community. For the clusters in our sample, we make publicly available both the catalog of probable and possible members (regardless of whether they have period measurements; Appendix C), as well as the catalog of the periodic stars (regardless of their membership classification; Appendix F). Additionally, we report the percentiles of the rotational distributions as a function of mass and age, which can be used to calibrate future models of angular momentum evolution (Appendix G).

In a broader sense, the field of stellar rotation is entering a new era thanks to the wealth of photometric and astrometric data provided by space-based missions (e.g., Kepler, K2, TESS, and Gaia). As shown in this work, these can be adequately complemented with ground-based observations. Still, although the rotational sequences of numerous starforming regions, stellar associations, and open clusters exist in the literature, properly accounting for their memberships remains a crucial part of exploiting them in full. Extensions of our work would increase the sample size and take advantage of improved astrometry and RV measurements (e.g., Gaia DR3) to perform full 6D kinematic analyses.

Ultimately, future rotation studies should attempt to answer the several questions that remain open. Besides the ones mentioned in Section 1, some of these include: the importance of shifts in magnetic field topology on the magnetic braking (Metcalfe \& Egeland 2019; Metcalfe et al. 2019); the role that bright faculae play in stellar variability (e.g., Montet et al. 2017; Reinhold \& Hekker 2020); and the deep connection between rotation and the various aspects of stellar activity (e.g., Stelzer et al. 2013; Dixon et al. 2020; Zhang et al. 2020; Godoy-Rivera et al. 2021; Ilin et al. 2021).
We thank Ângela Santos, Rafael García, and Savita Mathur for providing period measurements for the NGC 6811 stars. We also thank John Stauffer for useful discussions.

D.G.R. and M.H.P. acknowledge support from NASA grant 80NSSC19K0597.

This work has made use of data from the European Space Agency (ESA) mission Gaia (https://www.cosmos.esa.int/ gaia), processed by the Gaia Data Processing and Analysis Consortium (DPAC, https://www.cosmos.esa.int/web/gaia/ dpac/consortium). Funding for the DPAC has been provided by national institutions, in particular the institutions participating in the Gaia Multilateral Agreement.

This publication makes use of data products from the Two Micron All Sky Survey, which is a joint project of the University of Massachusetts and the Infrared Processing and Analysis Center/California Institute of Technology, funded by the National Aeronautics and Space Administration and the National Science Foundation.

\section{Appendix}

In Appendix A, we discuss the systems that were discarded from this analysis but that could be studied in future works. In Appendix B, we report the astrometric cluster parameters we derive, and compare them with the literature values. In Appendix C, we report a table with the Gaia DR2 information for the probable and possible cluster members (independently of whether they belong to the periodic sample or not). In Appendix D, we show the phase-space projections of the probable, possible, and non members for all the clusters, and provide a brief astrometric analysis for each of them. In Appendix E, we show the comparison of our mass and temperature values with independent estimates from the literature. In Appendix F, we report a table with the main information and properties for the stars in the periodic samples. In Appendix G, we report the percentiles of the rotation period distributions as a function of mass and age.

\section{Appendix A \\ Potential Expansions to Our Cluster Sample}

There are a number of systems that we initially considered including in our sample given their high scientific interest, but that we discarded for specific reasons, which we discuss below. We leave an equivalent investigation of them as future work to be done with a more sophisticated underlying model and when higher-precision astrometry becomes available.

1. $\rho$ Ophiuchus, Taurus, Upper Scorpius, and h Per: Rebull et al. (2018, 2020) and Moraux et al. (2013) reported periods for these young associations (ages of $\sim 1,3,8$, and $13 \mathrm{Myr}$, respectively). Given their young ages, these systems offer interesting pre-main-sequence stellar rotation constraints. For the first three of these, however, their more complex kinematics and reddening distributions would likely require somewhat different analysis techniques. For $\mathrm{h}$ Per, on the other hand, its small parallax ( $\approx 0.399$ mas; Cantat-Gaudin et al. 2018) and the current precision of the faint star Gaia data precludes a thorough examination. Therefore, we defer the consideration of these systems to a subsequent paper. 
2. IC $2391 / 2602$ and $\alpha$ Per: with ages of $\sim 50$ and $\sim 80 \mathrm{Myr}$ respectively, these clusters offer snapshots of the evolution of stellar rotation at young ages that could be compared with slightly younger and older systems (e.g., NGC 2547 and the Pleiades). Unfortunately, the rotation samples for these systems are still small ( $<40$ stars each) and heterogeneous (Denissenkov et al. 2010; Gallet \& Bouvier 2015).

3. M34: rotation periods for stars in this $\sim 220 \mathrm{Myr}$ old cluster have been reported by Irwin et al. (2006) (as one of the Monitor Project targets), James et al. (2010), and Meibom et al. (2011b). Although we initially attempted to include M34 in our sample, the Gaia data of this system showed odd gaps in spatial and magnitude space, possibly related to problems with the scanning pattern of this region of the sky. This prevented us from carrying out a similar study for this cluster at this time.

4. Hyades: rotation periods for stars in this $\sim 625 \mathrm{Myr}$ old cluster have been reported by a number of authors (e.g.,
Delorme et al. 2011; Douglas et al. 2016, 2019), and the Hyades is sufficiently close $(\approx 47 \mathrm{pc}$; Gaia Collaboration et al. 2018a) and well-studied that we do not anticipate substantial membership issues.

5. NGC 6819: rotation periods for 30 stars in this cluster were reported by Meibom et al. (2015). Given its age of $\sim 2$ Gyr (Bossini et al. 2019), studying this cluster is of high scientific interest. However, given its small parallax ( $\approx 0.356$ mas; Cantat-Gaudin et al. 2018), and the current precision of the Gaia data, this cluster would naturally be harder to fit with our current method.

\section{Appendix B \\ Astrometric Cluster Parameters and Comparison with the Literature}

Using the method described in Section 3.1, we calculate the astrometric parameters for the seven clusters we study. These values are reported in Table B1. We compare our cluster
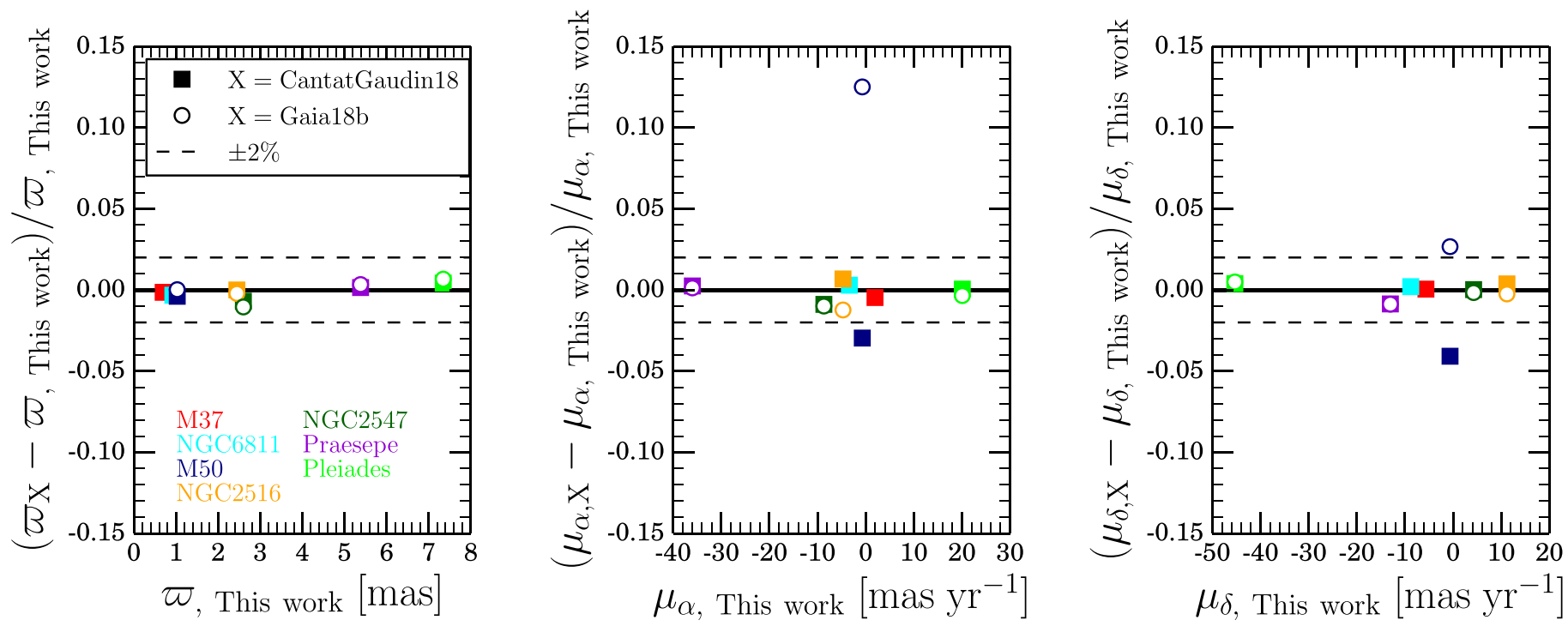

Figure B1. Comparison of our cluster parameters with literature reference values from Cantat-Gaudin et al. (2018) and Gaia Collaboration et al. (2018a). Left Panel: parallax difference divided by our parallax value, as a function of our parallax value. The comparison with Cantat-Gaudin et al. (2018) is shown as the filled squares, while the comparison with Gaia Collaboration et al. (2018a) is shown as the open circles. Each of the seven clusters we study is plotted with a different color, and this is indicated by the appropriate text near the bottom. The black dashed line shows a $\pm 2 \%$ fractional difference for reference. To make an accurate parallax comparison, we are adding the same zero-point value to the parallax of all three sources. Middle and Right Panels: same as the left panel for $\mu_{\alpha}$ and $\mu_{\delta}$. Note that Gaia Collaboration et al. (2018a) do not report cluster parameters for M37 and NGC 6811.

Table B1

Astrometric Cluster Parameters

\begin{tabular}{lccccr}
\hline \hline Cluster Name & $\begin{array}{c}\varpi \\
(\mathrm{mas})\end{array}$ & $\begin{array}{c}\sigma_{\varpi} \\
(\mathrm{mas})\end{array}$ & $\begin{array}{c}\mu_{\alpha} \\
\left(\mathrm{mas} \mathrm{yr}^{-1}\right)\end{array}$ & $\begin{array}{c}\sigma_{\mu_{\alpha}} \\
\left(\mathrm{mas} \mathrm{yr}^{-1}\right)\end{array}$ & $\begin{array}{c}\mu_{\delta} \\
\left(\mathrm{mas} \mathrm{yr}^{-1}\right)\end{array}$ \\
\hline NGC 2547 & 2.600 & 0.072 & -8.686 & 0.550 & 4.261 \\
Pleiades & 7.344 & 0.269 & 20.066 & 1.505 & -45.325 \\
M50 & 1.030 & 0.023 & -0.709 & 0.257 & -0.637 \\
NGC 2516 & 2.446 & 0.065 & -4.716 & 0.592 & 11.179 \\
M37 & 0.696 & 0.031 & 1.933 & 0.184 & -5.645 \\
Praesepe & 5.382 & 0.120 & -36.001 & 1.171 & 0.1757 \\
NGC 6811 & 0.926 & 0.018 & -3.389 & 0.120 & -13.031 \\
\hline
\end{tabular}

Note. List of cluster parameters (parallax, proper motion in $\alpha$ and $\delta$, and their respective dispersions) for the seven clusters we study. These are the values we use in Equation (7) to calculate membership probabilities. Note that the reported parallax values include the global $29 \mu$ as zero-point offset described in Section 3.1.1, with the exception of NGC 6811, for which we use the Kepler field $53 \mu$ as zero-point value from Zinn et al. (2019). 
parameters with those reported by Cantat-Gaudin et al. (2018) and Gaia Collaboration et al. (2018a) in Figure B1.

We find an excellent agreement with the literature, with most of the fractional differences being less than $\pm 2 \%$. The only exceptions to this are the proper motion parameters of M50 (dark blue points in Figure B1). This is perhaps not surprising, as the M50 proper motion in both coordinates is quite small (and similar to that of the field). We would therefore naturally expect this system to show the largest differences. We nonetheless note that Cantat-Gaudin et al. (2018) and Gaia Collaboration et al. (2018a) also get the largest disagreements between their values for M50, and our parameters are in between their values.

\section{Appendix C} Data Table for the Probable and Possible Cluster Members

Table $\mathrm{C} 1$ lists the probable and possible cluster members for all the clusters we study. We report the main Gaia DR2 astrometric and photometric information, as well as the membership parameters and classifications we derive.

We caution that, while Table $\mathrm{C} 1$ reports the same set of values for all stars regardless their membership classification, the $M_{G_{0, \text { proj }}}$, mass, and $T_{\text {eff }}$ values are calculated assuming as if all the stars are true main-sequence cluster members (and are all at the same respective global cluster parallax). For stars that turn out not to be real members, this will naturally yield an

Table C1

Main Information and Properties for the Probable and Possible Cluster Members

\begin{tabular}{lll}
\hline \hline Column & Source & Description \\
\hline Cluster & This paper & Cluster with respect to which this star's membership was calculated \\
Gaia DR2 & Gaia DR2 Catalog & Gaia DR2 Source ID \\
$\alpha$ & Gaia DR2 Catalog & R.A. coordinate \\
$\delta$ & Gaia DR2 Catalog & Decl. coordinate \\
$\varpi$ & Gaia DR2 Catalog & Parallax (not including zero-point value) \\
$\sigma_{\varpi}$ & Gaia DR2 Catalog & Parallax error \\
$\mu_{\alpha}$ & Gaia DR2 Catalog & Proper motion in R.A. \\
$\sigma_{\mu_{\alpha}}$ & Gaia DR2 Catalog & Proper motion in R.A. error \\
$\mu_{\delta}$ & Gaia DR2 Catalog & Proper motion in decl. \\
$\sigma_{\mu_{\delta}}$ & Gaia DR2 Catalog & Proper motion in decl. error \\
$G$ & Gaia DR2 Catalog & $G$-band magnitude \\
$G_{\mathrm{BP}}$ & Gaia DR2 Catalog & $G_{\mathrm{BP}}$-band magnitude \\
$G_{\mathrm{RP}}$ & Gaia DR2 Catalog & $G_{\mathrm{RP}}$-band magnitude \\
$G_{\mathrm{BP}}-G_{\mathrm{RP}}$ & Gaia DR2 Catalog & $G_{\mathrm{BP}}-G_{\mathrm{RP}}$ color \\
$\Delta$ & This paper & $\Delta$ quantity (as defined in Equation (6)) \\
Probability & This paper & Membership probability (as defined in Equation (7)) \\
Classification & This paper & Membership classification (i.e., $p$ probable or $p$ ossible member) \\
$M_{G_{0, p r o j}}$ & This paper & Projected absolute and dereddened $G$-band magnitude \\
Mass & This paper & Mass obtained by interpolating the best-fit cluster model \\
$T_{\text {eff }}$ & This paper & Effective temperature obtained by interpolating the best-fit cluster model \\
Flag Mass and $T_{\text {eff }}$ & This paper & Regime in which the $M_{G_{0, p r o j}}$ mass, and $T_{\text {eff }}$ values were calculated \\
\hline
\end{tabular}

Note. We report the main Gaia DR2 astrometric and photometric information, as well as the membership parameters we calculate, for the stars we classify as probable and possible cluster members. We also report our derived projected absolute and dereddened $G$-band magnitudes $\left(M_{G_{0, \text { proj }}}\right)$, as well as the respective mass and temperature values obtained by interpolating the models, and the regime in which they were calculated (see Section 4.2 for details). The parallaxes are reported as they appear in Gaia DR2 and do not include the zero-point values we have used elsewhere. For abbreviation purposes, we only list the possible members with membership probabilities greater than $50 \%$. We are glad to provide extended tables (including the same information for all the possible members as well as for the non members and no info stars) to interested readers upon request.

(This table is available in its entirety in machine-readable form.) 
incorrect mass and $T_{\text {eff }}$ estimate. Therefore, we suggest that interested readers should only consider the mass and $T_{\text {eff }}$ values for the probable members as being meaningful in future studies, until higher-quality astrometry fully resolves the membership classification of the possible members reported here.

\section{Appendix D \\ Astrometric Analysis of Clusters and Phase-space Projections}

After applying our classification method to all the clusters, we obtain a sample of probable members, possible members, non members, and no info stars for each of them. Figure D1 shows the phase-space projections of these populations (except for the no info stars, which are absent from the plot due to the lack of astrometric information; see Section 3.1.3). We now provide an analysis of the astrometric classification in each of the clusters:

1. NGC 2547: for this cluster, the parallax and proper motion in R.A. are the variables that allow us to distinguish the cluster from the field population in phase space. On the other hand, the proper motion in decl. of the cluster is similar to that of the field. The possible members are an intermediate population located in between the cluster and the field in the phase-space projections.

2. Pleiades: of all the clusters in our sample, this cluster is the one that is the most separated from the field in phase space. Given its large parallax as well as proper motion (particularly in decl.), this cluster can be readily differentiated from the background population. Some of the probable members appear as outliers in the projections including parallax, but they correspond to faint stars (apparent $G \gtrsim 18.5 \mathrm{mag}$ ) with uncertain parallax measurements. The small number of stars classified as possible members is a direct consequence of the large kinematic differences between the cluster and the field. In other words, it is unlikely that a star will have a value of $\Delta_{i} \leqslant 3$ (inside the cluster's $3 \sigma$ ellipsoid in phase space) and will have a low cluster membership probability.

3. M50: in this case, the proper motion of the cluster is quite similar to that of the field, and therefore the parallax plays a crucial role in identifying the probable cluster members. Nonetheless, given the small cluster parallax $(\sim 1.0$ mas $)$ and the consequently larger associated astrometric uncertainties (as the stars in M50 are naturally fainter than those of, for instance, the Pleiades), together with the proper motion similarities with the field, the number of possible members for this cluster is much larger than for others. In other words, given the large astrometric uncertainties, there are a large number of stars with values of $\Delta_{i} \leqslant 3$ (inside the cluster's $3 \sigma$ ellipsoid) that have low membership probabilities $\left(P<P_{\min }\right)$. The CMD of this cluster (see Figure 5) demonstrates this, as for magnitudes fainter than apparent $G \simeq 17 \mathrm{mag}$, the probable members are surrounded by an envelope of possible members. Additionally, the proper motion projection shows that the possible members are overlapping with the non members altogether.

4. NGC 2516: for this cluster, the parallax plays an important role when identifying the probable members. Although there are differences between the cluster and field proper motions (more in the decl. coordinate than in R.A.), the parallax is the dominant means to separate the populations. As in the case of NGC 2547, the possible members appear as an intermediate population in the phase-space projections.

5. M37: this cluster has the smallest parallax of our entire sample, in addition to having a proper motion in R.A. rather similar to that of the field. Thus, the proper motion in decl. plays an important role in the astrometric classification. M37 is also the richest cluster in our sample, and we identify a large number of probable members that densely populate the CMD. Moreover, a few giant stars can be seen in the CMD, highlighting that our classification method is agnostic to the stars photometry, and they naturally arise from a careful astrometric selection. Similarly as for the Pleiades, the probable members that appear as phase-space outliers correspond to faint stars.

6. Praesepe: similarly to the Pleiades, this cluster is clearly separated from the field in phase space. Again, some of the probable members appear as outliers particularly in proper motion space, but they correspond to faint stars with large astrometric uncertainties. The small number of possible members is again a reflection of the large kinematic difference between the cluster and field.

7. NGC 6811: this cluster has a proper motion in R.A. similar to that of the field, and therefore the parallax and proper motion in decl. are the variables that allow us to better distinguish the probable cluster members from the non members. However, given its small parallax value (compared to other clusters), and similarly to M50, NGC 6811 also has a large number of stars classified as possible members that overlap with the non members in phase space (and moreover, for magnitudes fainter than apparent $G \simeq 19$ mag, the astrometric errors are so large that no star can be reliably classified as a probable member). 

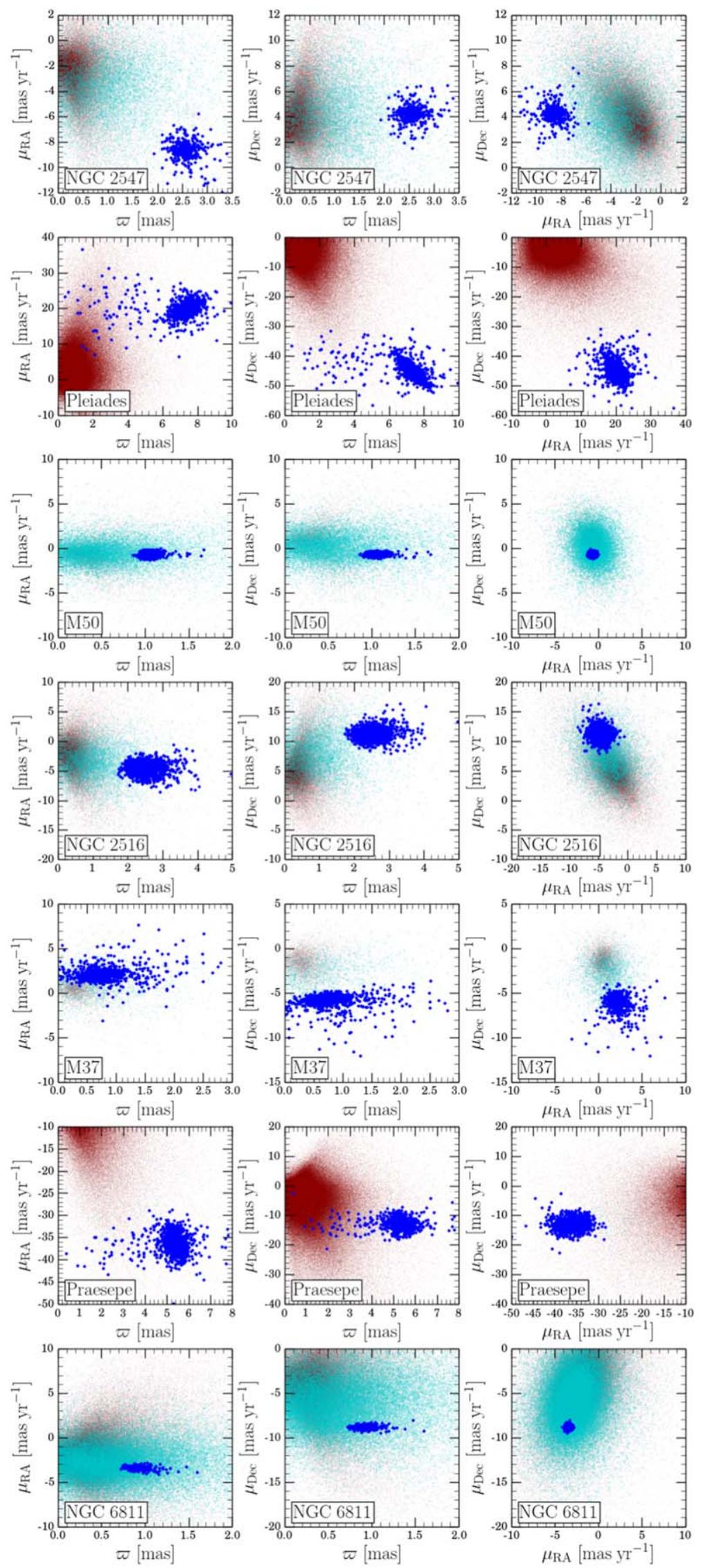

Figure D1. Phase-space projections of the probable (blue), possible (cyan), and non (red) members for all the clusters we study (analogous to Figure 2). Each cluster corresponds to a row, and they are sorted as presented in Table 1. Note that the range of the parallax and proper motions vary considerably on a cluster-by-cluster basis. 


\section{Appendix E \\ Comparison of Our Masses and Temperatures with Literature Values}

Analogous to the comparison made in Section 4.2 for NGC 2547 , some of the other clusters we study have mass (and sometimes $T_{\text {eff }}$ ) estimates available in the references that we use as sources for the period information. In particular, Irwin et al. $(2008,2009,2007 b)$ report masses for the NGC 2547, M50, and NGC 2516 stars, and Stauffer et al. (2016) and Curtis et al. (2019a) report mass and $T_{\text {eff }}$ for the Pleiades and NGC 6811 stars. We show the comparison of these estimates with ours in Figure E1. Note that, for M37 and Praesepe, the references used as sources for the period information did not report mass or temperature estimates, and these clusters are missing from the comparison.

The mass comparisons with NGC 2547, M50, and NGC 2516 are topologically similar to each other: in the faint regime (masses below the dashed lines), where we do not account for the contribution of photometric binaries, we see mostly constant differences of order $\approx-0.05 M_{\odot}$. For masses higher than this limit, the scatter increases and we observe that many stars have positive mass differences, which is due to the magnitude projections we use in the intermediate and bright regimes.

For the Pleiades, the mass and temperature comparisons with Stauffer et al. (2016) are in agreement with our expectations: while we employed the projection technique to account for unresolved binaries in our bright regime and partially in our intermediate regime, Stauffer et al. (2016) used this approach for their entire sample. Accordingly, we observe an approximately constant offset trend in $\Delta$ Mass $\left(\approx+0.05 M_{\odot}\right)$ and $\Delta T_{\text {eff }}$ $(\approx-150 \mathrm{~K})$ for stars more massive/hotter than the dotted line, which then turns to a mass $/ T_{\text {eff }}$-dependent trend for stars in the intermediate and faint regimes.

For NGC 6811, Curtis et al. (2019a) derived temperatures by generating their own color $-T_{\text {eff }}$ relations, and translated these to masses by interpolating tabulated spectral energy distributions. The mass and $T_{\text {eff }}$ comparisons show an excellent agreement, with differences being $\lesssim 0.025 M_{\odot}$ and $\lesssim 100 \mathrm{~K}$ for most of the mass $/ T_{\text {eff }}$ range. Given that both our and their calculations are based only on the Gaia DR2 photometry (unlike the previously described comparisons), this result is consistent with the expectations.

Ultimately, all the mass and temperature comparisons previously described are subject to different assumptions regarding the cluster properties, stellar evolutionary models (and their specific input physics; e.g., Tayar et al. 2020), and considerations for the contribution of unresolved photometric binaries. Furthermore, in most cases, the photometry employed by the references that supply the period information is different from that of Gaia. In spite of this, we observe a good overall agreement with the literature estimates, and these comparisons suggest possible systematic uncertainties of $\sim 0.05-0.1 M_{\odot}$ and $\sim 150 \mathrm{~K}$ in our masses and temperatures. 

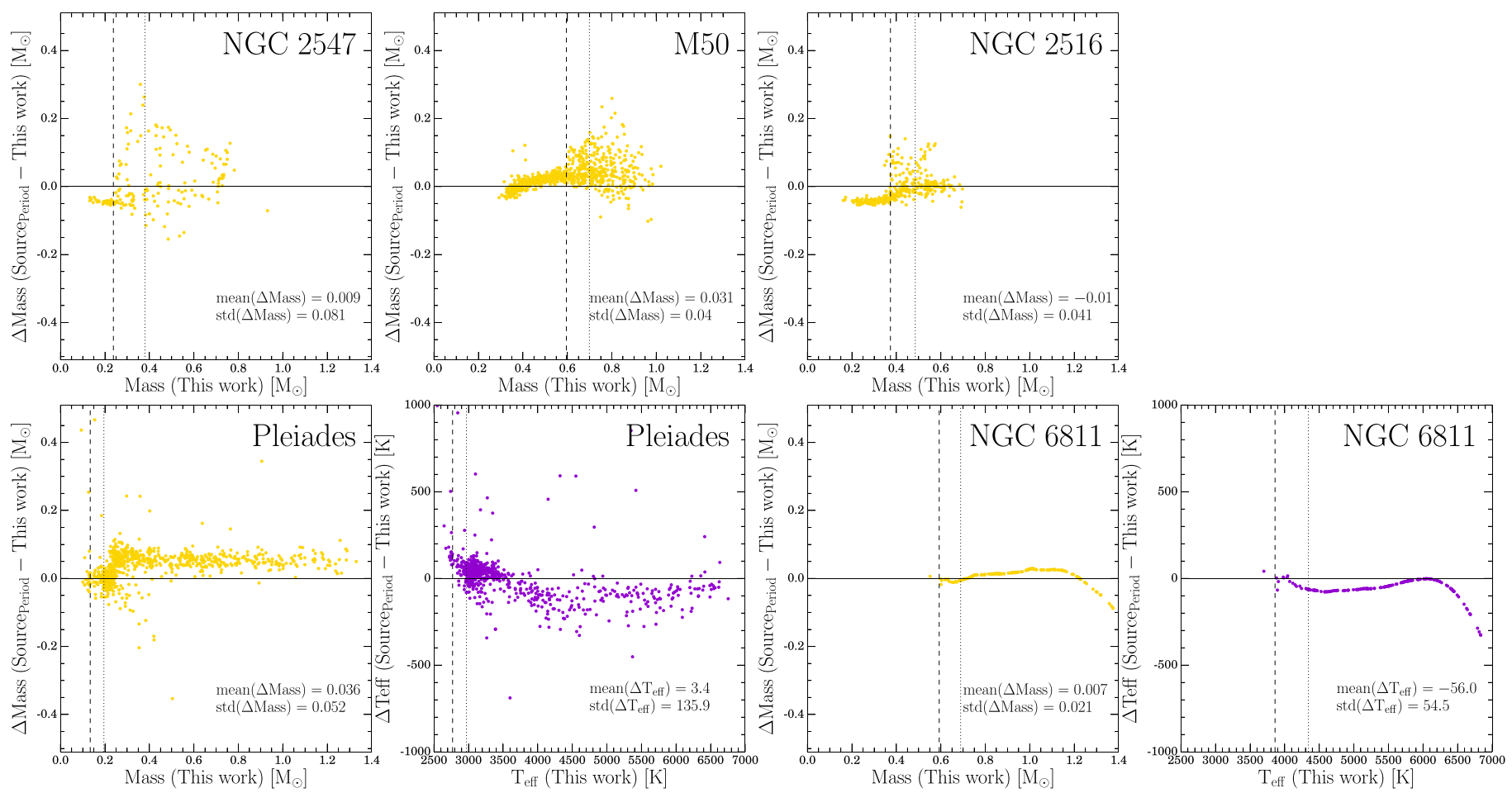

Figure E1. Comparison of our mass and $T_{\text {eff }}$ estimates with literature values (analogous to Figure 7). The mass comparisons are shown in yellow, while the $T_{\text {eff }}$ comparisons are shown in purple. The axis scale is the same for all the mass and $T_{\text {eff }}$ comparisons. Each panel indicates the respective cluster name in the top right corner and the mean and standard deviation of the mass or $T_{\text {eff }}$ difference in the bottom right corner. The dotted and dashed lines correspond to the apparent $G=17.5$ and 18.5 mag limits from Figure 5 translated to mass and $T_{\text {eff }}$ coordinates. The comparisons suggest that our masses and temperatures are subject to systematic uncertainties of $\sim 0.05-0.1 M_{\odot}$ and $\sim 150 \mathrm{~K}$, which are modest considering the different methods, photometric data, and underlying models employed.

\section{Appendix F \\ Data Table for the Periodic Samples}

Table F1 reports the main information and properties for the periodic samples of the clusters we study. We list all stars that were reported in the catalogs used as sources for the periods (see
Section 2), regardless of their membership classification or whether we found them when crossmatching with the Gaia DR2 data. We extend the same cautionary note given in Tables $\mathrm{C} 1$ to $\mathrm{F} 1$, regarding the fact that the $M_{G_{0, \text { proj }}}$, mass, and $T_{\text {eff }}$ values are calculated assuming as if all the stars are true main-sequence cluster members (and are all at the same respective global cluster parallax). 
Table F1

Main Information and Properties for the Periodic Samples

\begin{tabular}{|c|c|c|}
\hline Column & Source & Description \\
\hline Cluster & This paper & Cluster with respect to which this star's membership was calculated \\
\hline Source ID & Period Source Catalog & ID in the catalog used as source of the period information \\
\hline Period & Period Source Catalog & Period \\
\hline Gaia DR2 & Gaia DR2 Catalog & Gaia DR2 Source ID \\
\hline$\alpha$ & Gaia DR2 Catalog & R.A. coordinate \\
\hline$\delta$ & Gaia DR2 Catalog & Decl. coordinate \\
\hline$\varpi$ & Gaia DR2 Catalog & Parallax (not including zero-point value) \\
\hline$\sigma_{\varpi}$ & Gaia DR2 Catalog & Parallax error \\
\hline$\mu_{\alpha}$ & Gaia DR2 Catalog & Proper motion in R.A. \\
\hline$\sigma_{\mu_{\alpha}}$ & Gaia DR2 Catalog & Proper motion in R.A. error \\
\hline$\mu_{\delta}$ & Gaia DR2 Catalog & Proper motion in decl. \\
\hline$\sigma_{\mu_{\delta}}$ & Gaia DR2 Catalog & Proper motion in decl. error \\
\hline$G$ & Gaia DR2 Catalog & $G$-band magnitude \\
\hline$G_{\mathrm{BP}}$ & Gaia DR2 Catalog & $G_{\mathrm{BP}}$-band magnitude \\
\hline$G_{\mathrm{RP}}$ & Gaia DR2 Catalog & $G_{\mathrm{RP}}$-band magnitude \\
\hline$G_{\mathrm{BP}}-G_{\mathrm{RP}}$ & Gaia DR2 Catalog & $G_{\mathrm{BP}}-G_{\mathrm{RP}}$ color \\
\hline$\Delta$ & This paper & $\Delta$ quantity (as defined in Equation (6)) \\
\hline Probability & This paper & Membership probability (as defined in Equation (7)) \\
\hline Classification & This paper & Membership classification \\
\hline$M_{G_{0, \text { proj }}}$ & This paper & Projected absolute and dereddened $G$-band magnitude \\
\hline Mass & This paper & Mass obtained by interpolating the best-fit cluster model \\
\hline$T_{\text {eff }}$ & This paper & Effective temperature obtained by interpolating the best-fit cluster model \\
\hline Flag Mass and $T_{\text {eff }}$ & This paper & Regime in which the $M_{G_{0, \text { proj }}}$, mass, and $T_{\text {eff }}$ values were calculated \\
\hline
\end{tabular}

Note. From the catalogs used as source of the period measurements, we report the IDs used in them (which vary on a cluster-by-cluster basis) and the periods. We note that, although many of the Pleiades and Praesepe stars had more than one period reported, this table only lists their main value, i.e., $P 1$ from Rebull et al. (2016a, 2016b, 2017), respectively. From Gaia DR2, we report the main astrometric and photometric information, and the parallaxes are reported as they appear in Gaia DR2 and do not include the zero-point values we have used elsewhere. From our analysis, we report the membership probability and classification regardless of their category (i.e., probable, possible, or non member, or no info star). We also report our derived projected absolute and dereddened $G$-band magnitudes $\left(M_{G_{0, \text { proj }}}\right)$, as well as the respective mass and temperature values obtained by interpolating the models, and the regime in which they were calculated (see Section 4.2 for details).

(This table is available in its entirety in machine-readable form.)

\section{Appendix G \\ Percentiles of the Period Distributions}

Table G1 reports the percentiles of the distribution of rotation periods as a function of mass and age. Note that only the stars that survive our membership analysis have been considered (i.e., the stars of the revised rotational sequences; see Figure 10). These values can be used to calibrate and test future models of angular momentum evolution. 
Table G1

Percentiles of the Distribution of Rotation Periods as a Function of Mass and Age

\begin{tabular}{|c|c|c|c|c|c|c|c|c|c|c|c|c|c|c|c|c|c|c|}
\hline Cluster & $\begin{array}{l}\text { Age } \\
\text { (Myr) }\end{array}$ & $\begin{array}{l}\text { Mass } \\
\left(M_{\odot}\right)\end{array}$ & $N_{\star}$ & $\begin{array}{c}P_{10} \\
\text { (days) }\end{array}$ & $\begin{array}{l}\mathrm{MAD}_{10} \\
\text { (days) }\end{array}$ & $\begin{array}{c}\sigma_{10} \\
\text { (days) }\end{array}$ & $\begin{array}{c}P_{25} \\
\text { (days) }\end{array}$ & $\begin{array}{c}\mathrm{MAD}_{25} \\
\text { (days) }\end{array}$ & $\begin{array}{c}\sigma_{25} \\
\text { (days) }\end{array}$ & $\begin{array}{c}P_{50} \\
\text { (days) }\end{array}$ & $\begin{array}{c}\mathrm{MAD}_{50} \\
\text { (days) }\end{array}$ & $\begin{array}{c}\sigma_{50} \\
\text { (days) }\end{array}$ & $\begin{array}{c}P_{75} \\
\text { (days) }\end{array}$ & $\begin{array}{c}\mathrm{MAD}_{75} \\
\text { (days) }\end{array}$ & $\begin{array}{c}\sigma_{75} \\
\text { (days) }\end{array}$ & $\begin{array}{c}P_{90} \\
\text { (days) }\end{array}$ & $\begin{array}{l}\mathrm{MAD}_{90} \\
\text { (days) }\end{array}$ & $\begin{array}{c}\sigma_{90} \\
\text { (days) }\end{array}$ \\
\hline NGC 2547 & 35 & 0.2 & 34 & 0.318 & 0.091 & 0.035 & 0.471 & 0.101 & 0.024 & 0.628 & 0.212 & 0.036 & 0.886 & 0.13 & 0.032 & 1.161 & 0.218 & 0.084 \\
\hline NGC 2547 & 35 & 0.3 & 29 & 0.393 & 0.04 & 0.017 & 0.586 & 0.193 & 0.051 & 1.063 & 0.344 & 0.064 & 1.369 & 0.161 & 0.042 & 2.408 & 0.81 & 0.336 \\
\hline NGC 2547 & 35 & 0.4 & 24 & 0.39 & 0.152 & 0.069 & 0.78 & 0.317 & 0.092 & 1.447 & 0.747 & 0.152 & 5.024 & 2.873 & 0.829 & 8.045 & 0.747 & 0.341 \\
\hline NGC 2547 & 35 & 0.55 & 24 & 0.807 & 0.409 & 0.187 & 1.591 & 0.433 & 0.125 & 3.146 & 1.61 & 0.329 & 5.111 & 1.463 & 0.422 & 7.287 & 1.43 & 0.652 \\
\hline NGC 2547 & 35 & 0.8 & 21 & 0.287 & 0.06 & 0.029 & 1.219 & 0.922 & 0.285 & 4.41 & 1.197 & 0.261 & 5.221 & 0.268 & 0.083 & 5.56 & 0.192 & 0.094 \\
\hline Pleiades & 125 & 0.2 & 268 & 0.238 & 0.041 & 0.006 & 0.308 & 0.051 & 0.004 & 0.445 & 0.148 & 0.009 & 0.653 & 0.134 & 0.012 & 0.909 & 0.161 & 0.022 \\
\hline Pleiades & 125 & 0.3 & 132 & 0.457 & 0.068 & 0.013 & 0.611 & 0.139 & 0.017 & 0.837 & 0.283 & 0.025 & 1.241 & 0.322 & 0.04 & 2.011 & 0.262 & 0.051 \\
\hline Pleiades & 125 & 0.4 & 70 & 0.288 & 0.032 & 0.009 & 0.476 & 0.207 & 0.035 & 1.134 & 0.709 & 0.085 & 1.996 & 0.462 & 0.078 & 4.041 & 1.584 & 0.423 \\
\hline Pleiades & 125 & 0.5 & 40 & 0.327 & 0.022 & 0.008 & 0.597 & 0.277 & 0.062 & 2.153 & 1.56 & 0.247 & 3.734 & 1.333 & 0.298 & 7.434 & 0.674 & 0.238 \\
\hline Pleiades & 125 & 0.6 & 54 & 0.399 & 0.094 & 0.029 & 1.661 & 1.304 & 0.251 & 3.887 & 2.48 & 0.337 & 6.641 & 1.83 & 0.352 & 9.292 & 0.667 & 0.203 \\
\hline Pleiades & 125 & 0.7 & 35 & 0.394 & 0.048 & 0.018 & 0.56 & 0.199 & 0.047 & 5.002 & 3.235 & 0.547 & 7.479 & 0.889 & 0.212 & 8.44 & 0.158 & 0.06 \\
\hline Pleiades & 125 & 0.8 & 23 & 0.54 & 0.226 & 0.105 & 6.13 & 0.627 & 0.185 & 6.813 & 0.742 & 0.155 & 7.65 & 0.336 & 0.099 & 8.249 & 0.302 & 0.141 \\
\hline Pleiades & 125 & 0.9 & 28 & 0.831 & 0.142 & 0.06 & 4.031 & 1.374 & 0.367 & 5.444 & 0.548 & 0.103 & 5.933 & 0.22 & 0.059 & 6.154 & 0.005 & 0.002 \\
\hline Pleiades & 125 & 1 & 20 & 1.476 & 0.765 & 0.382 & 3.297 & 0.211 & 0.067 & 3.991 & 0.658 & 0.147 & 4.549 & 0.465 & 0.147 & 5.137 & 0.575 & 0.287 \\
\hline Pleiades & 125 & 1.1 & 18 & 1.116 & 0.279 & 0.147 & 2.138 & 0.259 & 0.086 & 2.453 & 0.455 & 0.107 & 3.081 & 0.552 & 0.184 & 3.907 & 0.334 & 0.176 \\
\hline Pleiades & 125 & 1.2 & 14 & 0.856 & 0.026 & 0.016 & 1.172 & 0.334 & 0.126 & 1.908 & 0.933 & 0.249 & 3.095 & 0.952 & 0.36 & 6.792 & 0.07 & 0.042 \\
\hline NGC 2516 & 150 & 0.2 & 27 & 0.291 & 0.038 & 0.017 & 0.37 & 0.027 & 0.007 & 0.413 & 0.068 & 0.013 & 0.561 & 0.101 & 0.027 & 2.136 & 1.487 & 0.64 \\
\hline NGC 2516 & 150 & 0.3 & 86 & 0.34 & 0.029 & 0.007 & 0.46 & 0.09 & 0.014 & 0.599 & 0.185 & 0.02 & 0.829 & 0.181 & 0.028 & 1.194 & 0.169 & 0.041 \\
\hline NGC 2516 & 150 & 0.4 & 110 & 0.364 & 0.056 & 0.012 & 0.605 & 0.236 & 0.032 & 1.179 & 0.541 & 0.052 & 1.685 & 0.414 & 0.056 & 2.987 & 0.709 & 0.151 \\
\hline NGC 2516 & 150 & 0.5 & 72 & 0.334 & 0.052 & 0.014 & 0.558 & 0.257 & 0.043 & 2.235 & 1.759 & 0.207 & 4.879 & 1.853 & 0.309 & 6.694 & 0.974 & 0.257 \\
\hline NGC 2516 & 150 & 0.6 & 49 & 0.446 & 0.039 & 0.012 & 1.282 & 0.95 & 0.192 & 4.971 & 3.227 & 0.461 & 7.756 & 1.268 & 0.256 & 9.606 & 0.908 & 0.29 \\
\hline M50 & 150 & 0.6 & 54 & 0.272 & 0.024 & 0.007 & 0.53 & 0.3 & 0.058 & 3.452 & 2.828 & 0.385 & 6.417 & 1.754 & 0.338 & 10.327 & 2.364 & 0.719 \\
\hline M50 & 150 & 0.7 & 106 & 0.456 & 0.111 & 0.024 & 1.116 & 0.844 & 0.116 & 5.627 & 3.191 & 0.31 & 7.753 & 1.104 & 0.152 & 9.522 & 0.666 & 0.145 \\
\hline M50 & 150 & 0.8 & 57 & 0.723 & 0.161 & 0.048 & 3.48 & 1.941 & 0.363 & 6.188 & 1.594 & 0.211 & 7.47 & 0.481 & 0.09 & 8.483 & 0.651 & 0.193 \\
\hline M50 & 150 & 0.9 & 46 & 1.29 & 0.854 & 0.282 & 3.633 & 1.137 & 0.237 & 4.992 & 1.541 & 0.227 & 6.723 & 0.923 & 0.192 & 7.868 & 0.626 & 0.206 \\
\hline M37 & 500 & 0.5 & 67 & 0.579 & 0.063 & 0.017 & 0.779 & 0.316 & 0.055 & 2.273 & 1.782 & 0.218 & 13.758 & 3.185 & 0.55 & 16.609 & 0.857 & 0.234 \\
\hline M37 & 500 & 0.6 & 78 & 1.292 & 0.352 & 0.089 & 2.208 & 1.073 & 0.172 & 8.665 & 5.491 & 0.622 & 13.801 & 1.147 & 0.184 & 15.055 & 0.473 & 0.12 \\
\hline M37 & 500 & 0.7 & 63 & 5.134 & 0.775 & 0.218 & 7.418 & 2.248 & 0.4 & 10.435 & 1.211 & 0.153 & 11.474 & 0.601 & 0.107 & 12.388 & 0.23 & 0.065 \\
\hline M37 & 500 & 0.8 & 58 & 7.512 & 0.401 & 0.118 & 8.242 & 0.429 & 0.08 & 9.06 & 0.821 & 0.108 & 9.902 & 0.377 & 0.07 & 10.303 & 0.189 & 0.056 \\
\hline M37 & 500 & 0.9 & 39 & 6.829 & 0.168 & 0.06 & 7.162 & 0.178 & 0.04 & 7.507 & 0.51 & 0.082 & 8.249 & 0.486 & 0.11 & 9.731 & 1.246 & 0.446 \\
\hline M37 & 500 & 1 & 18 & 5.372 & 0.356 & 0.188 & 6.37 & 0.354 & 0.118 & 6.817 & 0.272 & 0.064 & 7.01 & 0.175 & 0.058 & 8.514 & 1.166 & 0.615 \\
\hline M37 & 500 & 1.1 & 18 & 3.225 & 0.049 & 0.026 & 3.531 & 0.383 & 0.128 & 4.632 & 1.182 & 0.279 & 6.22 & 0.609 & 0.203 & 6.776 & 0.46 & 0.243 \\
\hline Praesepe & 700 & 0.2 & 132 & 0.254 & 0.026 & 0.005 & 0.331 & 0.09 & 0.011 & 0.635 & 0.331 & 0.029 & 1.138 & 0.364 & 0.045 & 1.806 & 0.194 & 0.038 \\
\hline Praesepe & 700 & 0.3 & 186 & 0.858 & 0.251 & 0.041 & 1.268 & 0.25 & 0.026 & 1.878 & 0.779 & 0.057 & 5.09 & 2.958 & 0.307 & 16.447 & 3.984 & 0.653 \\
\hline Praesepe & 700 & 0.4 & 86 & 0.902 & 0.122 & 0.029 & 3.25 & 2.352 & 0.359 & 12.682 & 8.758 & 0.944 & 20.605 & 4.221 & 0.644 & 25.071 & 1.267 & 0.306 \\
\hline Praesepe & 700 & 0.5 & 53 & 2.076 & 1.037 & 0.319 & 16.739 & 2.013 & 0.391 & 18.873 & 2.064 & 0.283 & 20.857 & 1.141 & 0.222 & 23.111 & 1.049 & 0.322 \\
\hline Praesepe & 700 & 0.6 & 63 & 7.529 & 3.363 & 0.948 & 14.365 & 0.846 & 0.151 & 15.454 & 1.133 & 0.143 & 16.732 & 0.682 & 0.121 & 18.07 & 0.645 & 0.182 \\
\hline Praesepe & 700 & 0.7 & 55 & 11.743 & 0.174 & 0.053 & 12.199 & 0.384 & 0.073 & 12.969 & 0.667 & 0.09 & 13.607 & 0.371 & 0.071 & 14.511 & 0.27 & 0.081 \\
\hline Praesepe & 700 & 0.8 & 29 & 9.368 & 0.807 & 0.335 & 10.421 & 0.249 & 0.066 & 10.886 & 0.552 & 0.103 & 11.556 & 0.26 & 0.068 & 12.073 & 0.257 & 0.107 \\
\hline Praesepe & 700 & 0.9 & 34 & 8.059 & 0.259 & 0.099 & 9.147 & 0.239 & 0.058 & 9.585 & 0.424 & 0.073 & 10.049 & 0.393 & 0.095 & 11.816 & 0.903 & 0.346 \\
\hline Praesepe & 700 & 1 & 30 & 4.489 & 1.513 & 0.618 & 8.048 & 0.557 & 0.144 & 8.899 & 0.472 & 0.086 & 9.088 & 0.107 & 0.028 & 10.268 & 0.642 & 0.262 \\
\hline Praesepe & 700 & 1.1 & 22 & 4.399 & 0.336 & 0.16 & 5.928 & 0.826 & 0.249 & 6.845 & 0.923 & 0.197 & 7.897 & 0.334 & 0.101 & 8.548 & 0.561 & 0.267 \\
\hline Praesepe & 700 & 1.2 & 14 & $\ldots$ & $\ldots$ & $\ldots$ & 3.122 & 0.497 & 0.188 & 3.745 & 0.969 & 0.259 & 5.077 & 0.443 & 0.168 & $\ldots$ & $\ldots$ & $\ldots$ \\
\hline Praesepe & 700 & 1.3 & 12 & $\cdots$ & $\cdots$ & $\cdots$ & 1.008 & 0.282 & 0.115 & 2.686 & 1.178 & 0.34 & 3.657 & 0.377 & 0.154 & $\ldots$ & $\ldots$ & $\ldots$ \\
\hline
\end{tabular}


Table G1

(Continued)

\begin{tabular}{|c|c|c|c|c|c|c|c|c|c|c|c|c|c|c|c|c|c|c|}
\hline Cluster & $\begin{array}{l}\text { Age } \\
\text { (Myr) }\end{array}$ & $\begin{array}{l}\text { Mass } \\
\left(M_{\odot}\right)\end{array}$ & $N_{\star}$ & $\begin{array}{c}P_{10} \\
\text { (days) }\end{array}$ & $\begin{array}{c}\mathrm{MAD}_{10} \\
\text { (days) }\end{array}$ & $\begin{array}{c}\sigma_{10} \\
\text { (days) }\end{array}$ & $\begin{array}{c}P_{25} \\
\text { (days) }\end{array}$ & $\begin{array}{c}\mathrm{MAD}_{25} \\
\text { (days) }\end{array}$ & $\begin{array}{c}\sigma_{25} \\
\text { (days) }\end{array}$ & $\begin{array}{c}P_{50} \\
\text { (days) }\end{array}$ & $\begin{array}{c}\mathrm{MAD}_{50} \\
\text { (days) }\end{array}$ & $\begin{array}{c}\sigma_{50} \\
\text { (days) }\end{array}$ & $\begin{array}{c}P_{75} \\
\text { (days) }\end{array}$ & $\begin{array}{c}\mathrm{MAD}_{75} \\
\text { (days) }\end{array}$ & $\begin{array}{c}\sigma_{75} \\
\text { (days) }\end{array}$ & $\begin{array}{c}P_{90} \\
\text { (days) }\end{array}$ & $\begin{array}{c}\mathrm{MAD}_{90} \\
\text { (days) }\end{array}$ & $\begin{array}{c}\sigma_{90} \\
\text { (days) }\end{array}$ \\
\hline Praesepe & 700 & 1.4 & 8 & $\ldots$ & $\ldots$ & $\ldots$ & 0.71 & 0.214 & 0.107 & 1.092 & 0.452 & 0.16 & 1.919 & 0.565 & 0.283 & $\ldots$ & $\ldots$ & $\ldots$ \\
\hline NGC 6811 & 950 & 0.6 & 12 & $\ldots$ & $\ldots$ & $\ldots$ & 13.983 & 0.403 & 0.164 & 14.43 & 0.375 & 0.108 & 14.97 & 0.54 & 0.22 & $\ldots$ & $\ldots$ & $\ldots$ \\
\hline NGC 6811 & 950 & 0.7 & 51 & 11.48 & 0.233 & 0.073 & 11.917 & 0.175 & 0.035 & 12.597 & 0.679 & 0.095 & 13.203 & 0.422 & 0.084 & 15.841 & 2.391 & 0.749 \\
\hline NGC 6811 & 950 & 0.8 & 36 & 7.82 & 2.626 & 0.978 & 10.591 & 0.256 & 0.06 & 10.966 & 0.447 & 0.074 & 11.672 & 0.705 & 0.166 & 12.859 & 0.349 & 0.13 \\
\hline NGC 6811 & 950 & 0.9 & 35 & 9.747 & 0.136 & 0.051 & 10.47 & 0.345 & 0.082 & 10.89 & 0.371 & 0.063 & 11.26 & 0.369 & 0.088 & 12.376 & 0.623 & 0.235 \\
\hline NGC 6811 & 950 & 1 & 25 & 9.555 & 0.009 & 0.004 & 9.918 & 0.305 & 0.086 & 10.303 & 0.588 & 0.118 & 10.929 & 0.482 & 0.136 & 13.007 & 0.253 & 0.113 \\
\hline NGC 6811 & 950 & 1.1 & 26 & 4.785 & 0.518 & 0.227 & 6.204 & 0.689 & 0.191 & 7.066 & 1.834 & 0.36 & 9.35 & 0.602 & 0.167 & 10.842 & 0.889 & 0.39 \\
\hline NGC 6811 & 950 & 1.2 & 26 & 1.472 & 0.122 & 0.054 & 2.022 & 0.278 & 0.077 & 2.828 & 0.824 & 0.162 & 3.726 & 0.763 & 0.212 & 7.434 & 2.693 & 1.181 \\
\hline NGC 6811 & 950 & 1.35 & 14 & 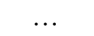 & $\ldots$ & $\ldots$ & 0.987 & 0.067 & 0.025 & 1.601 & 0.547 & 0.146 & 2.145 & 0.199 & 0.075 & 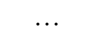 & $\ldots$ & $\ldots$ \\
\hline
\end{tabular}

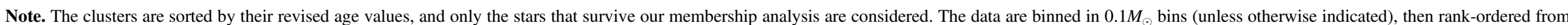

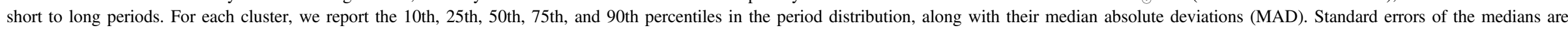

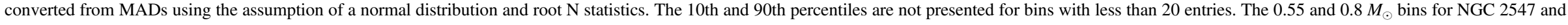
the $1.35 M_{\odot}$ bin for NGC 6811 are binned in broader bins $\left(0.45-0.70,0.7-0.9\right.$, and $1.25-1.45 M_{\odot}$, respectively) because of small sample sizes.

(This table is available in its entirety in machine-readable form.) 


\section{ORCID iDs}

Diego Godoy-Rivera @ https://orcid.org/0000-00034556-1277

Marc H. Pinsonneault 다 https://orcid.org/0000-00027549-7766

Luisa M. Rebull (ib https://orcid.org/0000-0001-6381-515X

\section{References}

Agüeros, M. A., Bowsher, E. C., Bochanski, J. J., et al. 2018, ApJ, 862, 33 Agüeros, M. A., Covey, K. R., Lemonias, J. J., et al. 2011, ApJ, 740, 110 Amard, L., \& Matt, S. P. 2020, ApJ, 889, 108

Amard, L., Roquette, J., \& Matt, S. P. 2020, MNRAS, 499, 3481

Anderson, C. M., Stoeckly, R., \& Kraft, R. P. 1966, ApJ, 143, 299

Angus, R., Aigrain, S., Foreman-Mackey, D., \& McQuillan, A. 2015, MNRAS, 450, 1787

Angus, R., Beane, A., Price-Whelan, A. M., et al. 2020, AJ, 160, 90

Angus, R., Morton, T. D., Foreman-Mackey, D., et al. 2019, AJ, 158, 173

Arenou, F., Luri, X., Babusiaux, C., et al. 2018, A\&A, 616, A17

Baraffe, I., Chabrier, G., Allard, F., \& Hauschildt, P. H. 1998, A\&A, 337, 403

Baraffe, I., Homeier, D., Allard, F., \& Chabrier, G. 2015, A\&A, 577, A42

Barnes, S. A. 2003, ApJL, 586, L145

Barnes, S. A. 2007, ApJ, 669, 1167

Belokurov, V., Penoyre, Z., Oh, S., et al. 2020, MNRAS, 496, 1922

Borucki, W. J., Koch, D., Basri, G., et al. 2010, Sci, 327, 977

Bossini, D., Vallenari, A., Bragaglia, A., et al. 2019, A\&A, 623, A108

Bouma, L. G., Curtis, J. L., Hartman, J. D., Winn, J. N., \& Bakos, G. Á. 2021, AJ, 162, 197

Bressan, A., Marigo, P., Girardi, L., et al. 2012, MNRAS, 427, 127

Cantat-Gaudin, T., Jordi, C., Vallenari, A., et al. 2018, A\&A, 618, A93

Cardelli, J. A., Clayton, G. C., \& Mathis, J. S. 1989, ApJ, 345, 245

Carmichael, T. W., Quinn, S. N., Zhou, G., et al. 2021, AJ, 161, 97

Chan, V. C., \& Bovy, J. 2020, MNRAS, 493, 4367

Chanamé, J., \& Ramírez, I. 2012, ApJ, 746, 102

Chen, Y., Girardi, L., Bressan, A., et al. 2014, MNRAS, 444, 2525

Choi, J., Dotter, A., Conroy, C., et al. 2016, ApJ, 823, 102

Clausen, J. V., Bruntt, H., Claret, A., et al. 2009, A\&A, 502, 253

Claytor, Z. R., van Saders, J. L., Santos, Â. R. G., et al. 2020, ApJ, 888, 43

Cummings, J. D., \& Kalirai, J. S. 2018, AJ, 156, 165

Cummings, J. D., Kalirai, J. S., Tremblay, P. E., \& Ramirez-Ruiz, E. 2016, ApJ, 818, 84

Cummings, J. D., Kalirai, J. S., Tremblay, P. E., Ramirez-Ruiz, E., \& Choi, J. 2018, ApJ, 866, 21

Curtis, J. L., Agüeros, M. A., Douglas, S. T., \& Meibom, S. 2019a, ApJ, 879,49

Curtis, J. L., Agüeros, M. A., Mamajek, E. E., Wright, J. T., \& Cummings, J. D. 2019b, AJ, 158, 77

Curtis, J. L., Agüeros, M. A., Matt, S. P., et al. 2020, ApJ, 904, 140

Davenport, J. R. A. 2017, ApJ, 835, 16

Davenport, J. R. A., \& Covey, K. R. 2018, ApJ, 868, 151

David, T. J., Contardo, G., Sandoval, A., et al. 2021, AJ, 161, 265

de Freitas, D. B. 2021, EL, 135, 19001

Delorme, P., Collier Cameron, A., Hebb, L., et al. 2011, MNRAS, 413, 2218

Denissenkov, P. A., Pinsonneault, M., Terndrup, D. M., \& Newsham, G. 2010, ApJ, 716, 1269

Dickens, R. J., Kraft, R. P., \& Krzeminski, W. 1968, AJ, 73, 6

Dixon, D., Tayar, J., \& Stassun, K. G. 2020, AJ, 160, 12

Dotter, A. 2016, ApJS, 222, 8

Douglas, S. T., Agüeros, M. A., Covey, K. R., et al. 2014, ApJ, 795, 161

Douglas, S. T., Agüeros, M. A., Covey, K. R., et al. 2016, ApJ, 822, 47

Douglas, S. T., Curtis, J. L., Agüeros, M. A., et al. 2019, ApJ, 879, 100

Francic, S. P. 1989, AJ, 98, 888

Franciosini, E., Sacco, G. G., Jeffries, R. D., et al. 2018, A\&A, 616, L12

Fritzewski, D. J., Barnes, S. A., James, D. J., \& Strassmeier, K. G. 2020, A\&A, 641, A51

Gaia Collaboration, Babusiaux, C., van Leeuwen, F., et al. 2018a, A\&A, 616, A10

Gaia Collaboration, Brown, A. G. A., Vallenari, A., et al. 2018b, A\&A, 616, A1

Gaia Collaboration, Brown, A. G. A., Vallenari, A., et al. 2021, A\&A, 649, A1

Gagné, J., \& Faherty, J. K. 2018, ApJ, 862, 138

Gallet, F. 2020, A\&A, 641, A38

Gallet, F., \& Bouvier, J. 2013, A\&A, 556, A36

Gallet, F., \& Bouvier, J. 2015, A\&A, 577, A98
Gallet, F., \& Delorme, P. 2019, A\&A, 626, A120

Galli, P. A. B., Joncour, I., \& Moraux, E. 2018, MNRAS, 477, L50

García, R. A., Ceillier, T., Salabert, D., et al. 2014, A\&A, 572, A34

Garraffo, C., Drake, J. J., Dotter, A., et al. 2018, ApJ, 862, 90

Godoy-Rivera, D., \& Chanamé, J. 2018, MNRAS, 479, 4440

Godoy-Rivera, D., Tayar, J., Pinsonneault, M. H., et al. 2021, ApJ, 915, 19

Gossage, S., Dotter, A., Garraffo, C., et al. 2021, ApJ, 912, 65

Gruner, D., \& Barnes, S. A. 2020, A\&A, 644, A16

Hall, O. J., Davies, G. R., van Saders, J., et al. 2021, NatAs, 5, 707

Hartman, J. D., Gaudi, B. S., Holman, M. J., et al. 2008a, ApJ, 675, 1254

Hartman, J. D., Gaudi, B. S., Holman, M. J., et al. 2008b, ApJ, 675, 1233

Hartman, J. D., Gaudi, B. S., Holman, M. J., et al. 2009a, ApJ, 695, 336

Hartman, J. D., Gaudi, B. S., Pinsonneault, M. H., et al. 2009b, ApJ, 691, 342

Healy, B. F., \& McCullough, P. R. 2020, ApJ, 903, 99

Herbst, W., Bailer-Jones, C. A. L., Mundt, R., Meisenheimer, K., \& Wackermann, R. 2002, A\&A, 396, 513

Howell, S. B., Sobeck, C., Haas, M., et al. 2014, PASP, 126, 398

Hurley, J., \& Tout, C. A. 1998, MNRAS, 300, 977

Ilin, E., Schmidt, S. J., Poppenhäger, K., et al. 2021, A\&A, 645, A42

Irwin, J., Aigrain, S., Bouvier, J., et al. 2009, MNRAS, 392, 1456

Irwin, J., Aigrain, S., Hodgkin, S., et al. 2006, MNRAS, 370, 954

Irwin, J., Aigrain, S., Hodgkin, S., et al. 2007a, MNRAS, 380, 541

Irwin, J., Berta, Z. K., Burke, C. J., et al. 2011, ApJ, 727, 56

Irwin, J., Hodgkin, S., Aigrain, S., et al. 2007b, MNRAS, 377, 741

Irwin, J., Hodgkin, S., Aigrain, S., et al. 2008, MNRAS, 383, 1588

Jackson, R. J., Deliyannis, C. P., \& Jeffries, R. D. 2018, MNRAS, 476, 3245

Jackson, R. J., Jeffries, R. D., Deliyannis, C. P., Sun, Q., \& Douglas, S. T. 2019, MNRAS, 483, 1125

James, D. J., Barnes, S. A., Meibom, S., et al. 2010, A\&A, 515, A100

Janes, K. A. 2017, ApJ, 835, 75

Jeffries, R. D., \& Oliveira, J. M. 2005, MNRAS, 358, 13

Jones, B. F., \& Prosser, C. F. 1996, AJ, 111, 1193

Jones, B. F., \& Stauffer, J. R. 1991, AJ, 102, 1080

Jones, B. F., \& Walker, M. F. 1988, AJ, 95, 1755

Jönsson, H., Holtzman, J. A., Allende Prieto, C., et al. 2020, AJ, 160, 120

Kawaler, S. D. 1988, ApJ, 333, 236

Kraft, R. P. 1967a, ApJ, 150, 551

Kraft, R. P. 1967b, ApJ, 148, 129

Kraus, A. L., Tucker, R. A., Thompson, M. I., Craine, E. R., \& Hillenbrand, L. A. 2011, ApJ, 728, 48

Kuhn, M. A., Hillenbrand, L. A., Sills, A., Feigelson, E. D., \& Getman, K. V. 2019, ApJ, 870, 32

Lehtinen, J. J., Spada, F., Käpylä, M. J., Olspert, N., \& Käpylä, P. J. 2020, NatAs, 4, 658

Lindegren, L., Hernández, J., Bombrun, A., et al. 2018, A\&A, 616, A2

Lodieu, N., Pérez-Garrido, A., Smart, R. L., \& Silvotti, R. 2019a, A\&A, 628, A66

Lodieu, N., Smart, R. L., Pérez-Garrido, A., \& Silvotti, R. 2019b, A\&A, 623, A35

Lorenzo-Oliveira, D., Meléndez, J., Yana Galarza, J., et al. 2019, MNRAS, 485, L68

Lurie, J. C., Vyhmeister, K., Hawley, S. L., et al. 2017, AJ, 154, 250

Magrini, L., Randich, S., Kordopatis, G., et al. 2017, A\&A, 603, A2

Majewski, S. R., Schiavon, R. P., Frinchaboy, P. M., et al. 2017, AJ, 154, 94

Mamajek, E. E., \& Hillenbrand, L. A. 2008, ApJ, 687, 1264

Matt, S. P., Brun, A. S., Baraffe, I., Bouvier, J., \& Chabrier, G. 2015, ApJL, 799, L23

McQuillan, A., Aigrain, S., \& Mazeh, T. 2013, MNRAS, 432, 1203

McQuillan, A., Mazeh, T., \& Aigrain, S. 2014, ApJS, 211, 24

Meibom, S., Barnes, S. A., Latham, D. W., et al. 2011a, ApJL, 733, L9

Meibom, S., Barnes, S. A., Platais, I., et al. 2015, Natur, 517, 589

Meibom, S., Mathieu, R. D., Stassun, K. G., Liebesny, P., \& Saar, S. H. 2011 b, ApJ, 733, 115

Messina, S. 2019, A\&A, 627, A97

Metcalfe, T. S., \& Egeland, R. 2019, ApJ, 871, 39

Metcalfe, T. S., Kochukhov, O., Ilyin, I. V., et al. 2019, ApJL, 887, L38

Miller, A. A., Irwin, J., Aigrain, S., Hodgkin, S., \& Hebb, L. 2008, MNRAS, 387, 349

Montet, B. T., Tovar, G., \& Foreman-Mackey, D. 2017, ApJ, 851, 116

Moraux, E., Artemenko, S., Bouvier, J., et al. 2013, A\&A, 560, A13

Naylor, T., \& Jeffries, R. D. 2006, MNRAS, 373, 1251

Netopil, M., Paunzen, E., Heiter, U., \& Soubiran, C. 2016, A\&A, 585, A150

Newton, E. R., Irwin, J., Charbonneau, D., et al. 2016, ApJ, 821, 93

Otani, T., von Hippel, T., Buzasi, D., Oswalt, T. D., \& Stone-Martinez, A. 2021, arXiv:2105.07266

Parker, E. N. 1958, ApJ, 128, 664

Pinsonneault, M. H., Kawaler, S. D., Sofia, S., \& Demarque, P. 1989, ApJ, 338,424 
Queloz, D., Allain, S., Mermilliod, J. C., Bouvier, J., \& Mayor, M. 1998, A\&A, 335, 183

Rebull, L. M., Stauffer, J. R., Bouvier, J., et al. 2016a, AJ, 152, 113

Rebull, L. M., Stauffer, J. R., Bouvier, J., et al. 2016b, AJ, 152, 114

Rebull, L. M., Stauffer, J. R., Cody, A. M., et al. 2018, AJ, 155, 196

Rebull, L. M., Stauffer, J. R., Cody, A. M., et al. 2020, AJ, 159, 273

Rebull, L. M., Stauffer, J. R., Hillenbrand, L. A., et al. 2017, ApJ, 839, 92

Reinhold, T., \& Hekker, S. 2020, A\&A, 635, A43

Ricker, G. R., Winn, J. N., Vanderspek, R., et al. 2015, JATIS, 1, 014003

Riello, M., De Angeli, F., Evans, D. W., et al. 2018, A\&A, 616, A3

Riello, M., De Angeli, F., Evans, D. W., et al. 2021, A\&A, 649, A3

Riess, A. G., Casertano, S., Yuan, W., et al. 2018, ApJ, 861, 126

Roccatagliata, V., Franciosini, E., Sacco, G. G., Randich, S., \& Sicilia-Aguilar, A. 2020, A\&A, 638, A85

Roccatagliata, V., Sacco, G. G., Franciosini, E., \& Randich, S. 2018, A\&A, 617, L4

Rodríguez, E., Balona, L. A., López-González, M. J., et al. 2020, MNRAS, 491,4345

Sanders, W. L. 1971, A\&A, 14, 226

Santos, A. R. G., Breton, S. N., Mathur, S., \& García, R. A. 2021, ApJS, 255, 17

Santos, A. R. G., García, R. A., Mathur, S., et al. 2019, ApJS, 244, 21

Schatzman, E. 1962, AnAp, 25, 18

Scholz, A., \& Eislöffel, J. 2007, MNRAS, 381, 1638

Schönrich, R., McMillan, P., \& Eyer, L. 2019, MNRAS, 487, 3568

Simonian, G. V. A., Pinsonneault, M. H., \& Terndrup, D. M. 2019, ApJ, 871,174

Simonian, G. V. A., Pinsonneault, M. H., Terndrup, D. M., \& van Saders, J. L. 2020, ApJ, 898, 76

Skumanich, A. 1972, ApJ, 171, 565

Smart, R. L., Marocco, F., Sarro, L. M., et al. 2019, MNRAS, 485, 4423

Soderblom, D. R. 2010, ARA\&A, 48, 581

Somers, G., Cao, L., \& Pinsonneault, M. H. 2020, ApJ, 891, 29

Somers, G., \& Pinsonneault, M. H. 2015, ApJ, 807, 174

Somers, G., \& Stassun, K. G. 2017, AJ, 153, 101
Somers, G., Stauffer, J., Rebull, L., Cody, A. M., \& Pinsonneault, M. 2017, ApJ, 850, 134

Spada, F., \& Lanzafame, A. C. 2020, A\&A, 636, A76

Spina, L., Randich, S., Magrini, L., et al. 2017, A\&A, 601, A70

Stassun, K. G., \& Torres, G. 2018, ApJ, 862, 61

Stauffer, J., Rebull, L., Bouvier, J., et al. 2016, AJ, 152, 115

Stauffer, J., Rebull, L. M., Cody, A. M., et al. 2018, AJ, 156, 275

Stauffer, J. R., Hartmann, L., Soderblom, D. R., \& Burnham, N. 1984, ApJ, 280, 202

Stauffer, J. R., \& Hartmann, L. W. 1987, ApJ, 318, 337

Stauffer, J. R., Schultz, G., \& Kirkpatrick, J. D. 1998, ApJL, 499, L199

Stelzer, B., Marino, A., Micela, G., López-Santiago, J., \& Liefke, C. 2013, MNRAS, 431, 2063

Tayar, J., Claytor, Z. R., Huber, D., \& van Saders, J. 2020, arXiv:2012.07957

Terndrup, D. M., Krishnamurthi, A., Pinsonneault, M. H., \& Stauffer, J. R. 1999, AJ, 118, 1814

Terndrup, D. M., Stauffer, J. R., Pinsonneault, M. H., et al. 2000, AJ, 119,1303

Tokovinin, A., \& Briceño, C. 2018, AJ, 156, 138

Torres, G., Andersen, J., \& Giménez, A. 2010, A\&ARv, 18, 67

Torres, G., \& Ribas, I. 2002, ApJ, 567, 1140

van Saders, J. L., Ceillier, T., Metcalfe, T. S., et al. 2016, Natur, 529, 181

van Saders, J. L., \& Pinsonneault, M. H. 2013, ApJ, 776, 67

van Saders, J. L., Pinsonneault, M. H., \& Barbieri, M. 2019, ApJ, 872, 128

Vasilevskis, S., Klemola, A., \& Preston, G. 1958, AJ, 63, 387

Velloso, E. N., do Nascimento, J. D., Jr., \& Saar, S. H. 2020, ApJ, 900, 173

Weber, E. J., \& Davis, L., Jr. 1967, ApJ, 148, 217

Williams, J. P., \& Cieza, L. A. 2011, ARA\&A, 49, 67

Wright, N. J., Drake, J. J., Mamajek, E. E., \& Henry, G. W. 2011, ApJ, 743, 48

Wright, N. J., Newton, E. R., Williams, P. K. G., Drake, J. J., \& Yadav, R. K. 2018, MNRAS, 479, 2351

Zhang, J., Bi, S., Li, Y., et al. 2020, ApJS, 247, 9

Zhou, G., Quinn, S. N., Irwin, J., et al. 2021, AJ, 161, 2

Zinn, J. C., Pinsonneault, M. H., Huber, D., \& Stello, D. 2019, ApJ, 878, 136 